\title{
A THEORY FOR ORDINARY DIFFERENTIAL BOUNDARY PROBLEMS OF THE SECOND ORDER AND OF THE HIGHLY IRREGULAR TYPE
}

\author{
BY \\ RUDOLPH E. LANGER
}

1. Introduction. The boundary problems with which this discussion is concerned may be given in either the form

$$
\begin{aligned}
& u^{\prime \prime}(x)+\left\{p_{11}(x) \lambda+p_{10}(x)\right\} u^{\prime}(x) \\
& +\left\{p_{22}(x) \lambda^{2}+p_{21}(x) \lambda+p_{20}(x)\right\} u(x)=0, \\
& h_{i 1}(\lambda) u^{\prime}(a)+h_{i 2}(\lambda) u(a)+h_{i 3}(\lambda) u^{\prime}(b)+h_{i 4}(\lambda) u(b)=0, \quad i=1,2,
\end{aligned}
$$

in which the coefficient functions $p_{i k}(x)$ and the solution $u(x)$ are scalars, or in the form

$$
\begin{gathered}
\mathfrak{u}^{\prime}(x)=\left\{\lambda \mathfrak{B}_{1}(x)+\mathfrak{P}_{0}(x)\right\} \mathfrak{u}(x), \\
\mathfrak{S}_{a}(\lambda) \mathfrak{u}(a)+\mathfrak{S}_{b}(\lambda) \mathfrak{u}(b)=\mathfrak{o},
\end{gathered}
$$

in which the German capital letters designate matrices of the order two and the solution $\mathfrak{u}(x)$ is a vector, that is, a matrix of two rows and one column. In either form the parameter $\lambda$ is to be taken as complex and unbounded, while the variable $x$ is to be taken as real and on the finite interval $(a, b)$. On this interval the coefficients $p_{i k}(x)$, or the elements of the matrices $\mathfrak{B}_{k}(x)$, are assumed to be differentiable, and such that the functions $r(x)$ which, in the case of the system (1.1), satisfy the equation

$$
r^{2}(x)+p_{11}(x) r(x)+p_{22}(x)=0,
$$

or, in the case of the system (1.2) make the matrix

$$
\left\{\mathfrak{P}_{1}(x)-r(x) \Im\right\}
$$

singular, fulfill conditions to be stated below in $\S 2$. The coefficients $h_{i k}(\lambda)$ of the boundary relations in (1.1), or the elements of the matrices $\mathfrak{W}_{a}(\lambda)$ and $\mathfrak{S}_{b}(\lambda)$ in (1.2), as the case may be, are to be polynomials in $\lambda$ of any degree, and may, of course, in particular be constants.

Any boundary problem of this type is compatible either for all values of $\lambda$ or for no such value, or for a certain set of characteristic values which is finite or denumerably infinite. This discussion is primarily concerned with the latter case. With an infinite set of characteristic values, there exists, then,

Presented to the Society, September 2,1941 under the title $A$ theory for ordinary linear differential boundary problems of highly irregular type; received by the editors July 13, 1942. 
an associated set of characteristic solutions, and by familiar procedures an infinite series of these solutions may be associated with an "arbitrary" function or vector. The series is then designated as a formal expansion of that function or vector, and the latter is in turn designated as the generating element of the expansion.

Contingent upon the fulfillment of certain more or less general conditions by the generating element, the behavior of an expansion, that is, its divergence, convergence, summability, value, and so on, is essentially determined by the boundary problem itself, specifically by the character of the adjustment which maintains between the differential equation and the boundary relations. This adjustment has, therefore, been made the basis for a classification of boundary problems into categories which are identified by the designations "regular," "mildly irregular" and "highly irregular."

For boundary problems of the regular type a relatively complete and familiar theory exists. The formal expansions include the classical Fourier's series as special cases, and have, broadly speaking, the salient properties of these series. Thus, in particular, they converge to the value of the generating element in an appropriately conventional sense whenever this element is integrable over the fundamental interval, and is of bounded variation in some neighborhood of the point under consideration.

Though less has been written upon boundary problems of the mildly irregular type $\left.{ }^{1}\right)$, the state of their theory is roughly comparable with that of the theory of regular problems. In general the formal expansions are divergent, but are summable by means of familiar type to the values of the generating elements.

By contrast with this, nothing that may properly be referred to as a general theory has heretofore been given for boundary problems of the highly irregular type. In these the classical methods apparently lead into insurmountable difficulties, and simple examples show that these difficulties are not due to the methods alone. The literature on problems of this type is, therefore, scant. Only problems which are markedly specialized and symmetrical have been analyzed at all $\left({ }^{2}\right)$, and in them, even in the face of their specialization, the results obtained are only in slight measure comparable with those of the theory of regular problems. Existing discussions, far from

(1) Cf. M. H. Stone, Irregular differential systems of order two and the related expansion problems. Trans. Amer. Math. Soc. vol. 29 (1927) pp. 23-53. R. E. Langer, The expansion problem in the theory of ordinary linear differential systems, Trans. Amer. Math. Soc. vol. 31 (1929) pp. 868-906.

(2) Cf. J. W. Hopkins, Trans. Amer. Math. Soc. vol. 20 (1919) pp. 245-259. L. E. Ward, Trans. Amer. Math. Soc. vol. 29 (1927) pp. 716-745, ibid. vol. 32 (1930) pp. 544-557, ibid. vol. 34 (1933) pp. 417-434, Ann. of Math. (2) vol. 26 (1925) pp. 21-36, and Amer. J. Math. vol. 57 (1935) pp. 345-362. In all of these the differential equation of the problem is of a form included in $d^{n} u / d x^{n}+\left\{\lambda^{n}+\phi(x)\right\} u=0, n \geqq 3$. Also, J. I. Vass, Duke Math. J. vol. 2 (1936) pp. 151-165, in which the differential equation is $d^{2} u / d x^{2}-2 \lambda \cos (p \pi / q) \cdot d u / d x+\lambda^{2} u=0$. 
applying to such formal expansions as have arbitrary generating elements, have been restricted to cases in which these elements are analytic as functions of the complex variable, and beyond that are of certain distinctive and extremely special structures.

The present paper is based upon a wholly different mode of approach to the problem. Its method is, in brief, the imbedding of the highly irregular problem in a continuous family of boundary problems of which all other members are regular. The given problem is thus approached through limiting considerations applied to existing theory. It is found on this basis that a subclassification of the highly irregular problems into two virtual sub-categories is requisite. For problems of the first sub-category, and this includes all problems of the second order and highly irregular type that have been discussed heretofore at all, a theory is derived which is in many respects closely aligned with the existing theories for regular and mildly irregular problems. Though the expansions are non-convergent, they are shown to be summable, in certain specifically defined senses, to the values of the generating elements, whenever these latter fulfill conditions such as are familiarly imposed in the theory of Fourier's series. For problems of the second sub-category no results are derived, and it seems improbable that any expansion properties as conventionally understood inhere in problems of this type.

The discussion has been restricted to boundary problems of the second order. The motive for this, however, is to be sought only in the desire to keep the paper within its present bounds. The method set forth is evidently more generally applicable.

\section{Chapter 1}

\section{THE GIVEN BOUNDARY PROBLEM}

2. The normalization of the differential equation. The forms of the boundary problems (1.1) and (1.2) remain unchanged under any integral linear change of the independent variable $x$. Since a suitable change of this kind reduces the interval $(a, b)$ to the interval $(0,1)$, it may be assumed without loss of generality, that $a=0$ and $b=1$. In the following this will be done. To obviate the incidence of complications which are not germane to the matter essentially at issue, it will be assumed that on this interval the coefficients $p_{k l}(x), k=1,2 ; l=0,1,2$; if the boundary problem is given in the form (1.1), or the elements of the matrices $\mathfrak{P}_{1}(x)$ and $\mathfrak{P}_{0}(x)$, if the problem is given in the form (1.2), are differentiable to any desired order, or at least to such orders as may be effectively called for.

If the boundary problem as given is in the form (1.1), let $r_{1}(x)$ and $r_{2}(x)$ designate the roots of the equation (1.3), and let it be supposed that these roots remain distinct on the interval $(0,1)$. The equations

$$
\frac{q_{1}^{\prime}}{q_{1}}=-p_{10}-2 \frac{\phi^{\prime}}{\phi},
$$




$$
r_{1} \frac{q_{1}^{\prime}}{q_{1}}-r_{1}^{\prime}=p_{21}+p_{11} \frac{\phi^{\prime}}{\phi},
$$

then define the functions $q_{1}^{\prime} / q_{1}$ and $\phi^{\prime} / \phi$, and if $q_{2}$ is determined from the formula

$$
q_{1} q_{2}=-\left(p_{20}+p_{10} \frac{\phi^{\prime}}{\phi}+\frac{\phi^{\prime \prime}}{\phi}\right)
$$

it is found that the equation obtained from the system

$$
\begin{aligned}
& y_{1}^{\prime}(x)=\lambda r_{1}(x) y_{1}(x)+q_{1}(x) y_{2}(x), \\
& y_{2}^{\prime}(x)=q_{2}(x) y_{1}(x)+\lambda r_{2}(x) y_{2}(x),
\end{aligned}
$$

by the elimination of the function $y_{2}(x)$, is identical with that obtained from the differential equation in (1.1) by making the substitution $u(x)=\phi(x) y_{1}(x)$. This substitution, together with the first of the equations (2.1), reduces the boundary relations of (1.1) to the forms

$$
v_{i 1}(\lambda) y_{1}(0)+v_{i 2}(\lambda) y_{2}(0)+v_{i 3}(\lambda) y_{1}(1)+v_{i 4}(\lambda) y_{2}(1)=0, \quad i=1,2,
$$

in which the coefficients $v_{i l}(\lambda)$ are again polynomials in $\lambda$. The differential system (1.1) is thus reducible to the form (2.1), (2.2). This latter may be conveniently written in matrix form, thus

$$
\begin{gathered}
\mathfrak{y}^{\prime}(x)=\{\lambda \Re(x)+\mathfrak{Q}(x)\} \mathfrak{y}(x), \\
\mathfrak{B}^{(0)}(\lambda) \mathfrak{y}(0)+\mathfrak{B}^{(1)}(\lambda) \mathfrak{y}(1)=0,
\end{gathered}
$$

in which

$$
\begin{gathered}
\mathfrak{y}(x) \equiv\left(\begin{array}{l}
y_{1}(x) \\
y_{2}(x)
\end{array}\right), \quad \Re(x) \equiv\left(\begin{array}{cc}
r_{1}(x) & 0 \\
0 & r_{2}(x)
\end{array}\right), \quad \mathfrak{Q}(x)=\left(\begin{array}{cc}
0 & q_{1}(x) \\
q_{2}(x) & 0
\end{array}\right), \\
\mathfrak{B}^{(0)}(\lambda) \equiv\left(v_{i j}(\lambda)\right), \quad \mathfrak{B}^{(1)}(\lambda) \equiv\left(v_{i, j+2}(\lambda)\right)\left({ }^{3}\right) .
\end{gathered}
$$

If the boundary problem as given is in the form (1.2), let $r_{1}(x)$ and $r_{2}(x)$ be the roots of the determinant equation

$$
\left|\begin{array}{ll}
p_{11}^{(1)}(x)-r & p_{12}^{(1)}(x) \\
p_{21}^{(1)}(x) & p_{22}^{(1)}(x)-r
\end{array}\right|=0,
$$

in which $\left(p_{i j}^{(1)}(x)\right) \equiv \mathfrak{P}_{1}(x)$, and let it be supposed that these roots are distinct on the interval $(0,1)$. The nonsingular matrix $\mathfrak{T}(x)$ which fulfills the relation

$$
\mathfrak{P}_{1}(x) \mathfrak{T}(x)=\mathfrak{T}(x) \Re(x)
$$

then exists, and the substitution $\mathfrak{u}(x)=\mathfrak{T}(x) \mathfrak{w}(x)$ gives to the differential

(3) Throughout the paper German capital letters will be used to designate square matrices of order two. Lower case German letters will correspondingly be used to designate vectors of two components, and Latin letters to denote scalars. 
equation in (1.2) the form

$$
\mathfrak{w}^{\prime}(x)=\left\{\lambda \Re(x)+\mathfrak{B}_{2}(x)\right\} \mathfrak{w}(x),
$$

with

$$
\mathfrak{P}_{2}(x)=\mathfrak{T}^{-1}(x)\left\{\mathfrak{P}_{0}(x) \mathfrak{T}(x)-\mathfrak{T}^{\prime}(x)\right\} .
$$

The further substitution

$$
\mathfrak{w}(x)=\left(\delta_{i j} e^{\int p_{i i}^{(2)}(x)} d x\right) \mathfrak{y}(x)\left({ }^{4}\right),
$$

reduces the equation to the differential equation in (2.3), and in fact the entire boundary problem (1.2) to the form of (2.3). Since the problem, whether given in the form (1.1) or in the form (1.2) is thus reducible to (2.3), the further considerations may be confined to this latter form.

It has already been assumed that the functions $r_{1}(x)$ and $r_{2}(x)$ are distinct on the interval $(0,1)$. Further restrictions upon these functions, which are imposed in all existing theories of boundary problems (2.3) when the independent variable is real $(5)$, and which are now also to be imposed herewith upon the present discussion are the following:

Hypothesis 1. On the interval $(0,1)$ the functions $r_{1}(x), r_{2}(x)$, and $\left\{r_{1}(x)-r_{2}(x)\right\}$, are bounded from zero, and each of them is real except possibly for a constant complex factor.

There are essentially two types of configuration which conform to this hypothesis, namely:

Configuration 1,

$$
r_{j}(x) \equiv \sigma_{j \rho}(x), \quad j=1,2,
$$

in which $\sigma_{1}$ and $\sigma_{2}$ are constants different from zero with a ratio which is not real, and $\rho(x)$ is a real positive function which is bounded from zero; and

Configuration 2,

$$
r_{j}(x) \equiv \sigma \rho_{j}(x), \quad j=1,2,
$$

in which $\sigma$ is a non-vanishing constant, and $\rho_{1}(x), \rho_{2}(x)$, are real functions which are bounded from each other and from zero.

To this point no stipulation has been made as to the assignment of subscripts to the functions $r_{1}(x)$ and $r_{2}(x)$. It is convenient to assign the subscripts now and henceforth in such a way that in the event of the configuration 1 , the value of $\left\{\arg r_{1}(x)-\arg r_{2}(x)\right\}$ lies between 0 and $\pi$. If the relations

(4) Through the paper $\delta_{i j}=0$ if $i \neq j$ and $\delta_{i j}=1$ if $i=j$.

(5) For the theory when $x$ ranges over a region of the complex plane cf. R. E. Langer, The boundary problem of an ordinary linear differential system in the complex domain, Trans. Amer. Math. Soc. vol. 46 (1939) pp. 151-190. 


$$
\begin{aligned}
\Gamma_{j}(x) & \equiv \int_{0}^{x} r_{j}(x) d x, \\
\Gamma_{j} & =\Gamma_{j}(1),
\end{aligned}
$$

are used to define their left-hand members, it follows then in the event of configuration 1 , that

$$
0<\arg \Gamma_{1}-\arg \Gamma_{2}<\pi .
$$

In the event of the formulas (2.6) the value of $\left\{\arg r_{1}(x)-\arg r_{2}(x)\right\}$ is a multiple of $\pi$. By a suitable assignment of subscripts in this case of configuration 2 , one or the other of the sets of relations

$$
\begin{array}{ll}
\arg \Gamma_{1}-\arg \Gamma_{2}=0, & \left|\Gamma_{1}\right|>\left|\Gamma_{2}\right|, \quad \text { or } \\
\arg \Gamma_{1}-\arg \Gamma_{2}=\pi, & \left|\Gamma_{1}\right| \geqq\left|\Gamma_{2}\right|,
\end{array}
$$

may therefore be achieved. It will be assumed in the following that the subscripts have been so assigned.

3 . The boundary conditions. The components of the vector boundary relation of the system (2.3) are given explicitly in the equations (2.2), and in this, as has already been noted, each coefficient $\mathfrak{b}_{i l}(\lambda)$ is a polynomial in $\lambda$, which may in particular vanish. Individually these relations are, of course, not uniquely specific, since they may be replaced by any independent linear combinations of the two without any modification of the content of the conditions as a whole being thereby induced. In the vector form of the condition, as it appears in (2.3), such a replacement is accomplished by the multiplication of the relation on the left by some nonsingular matrix, and conversely any such multiplication by a nonsingular matrix is of merely formal effect.

If in either of the relations (2.2) all four coefficients $\mathfrak{v}_{i l}(\lambda), l=1,2,3,4$, have some factor $\left(\lambda-\lambda_{0}\right)$ in common, the boundary problem is compatible at $\lambda_{0}$. The same, but no more, follows if they have $\left(\lambda-\lambda_{0}\right)$ as a multiple common factor. A reduction of the multiplicity of such a factor is, therefore, a permissible formal simplification, and in proceeding it will be assumed that such simplifications have been made, so that any common factor of the coefficients $\mathfrak{b}_{i l}(\lambda)$, with $i=1$ or $i=2$, is a simple factor of at least one of them.

If the two relations (2.2) are linearly dependent identically in $\lambda$, the boundary problem is permanently compatible, and, from the point of view of this discussion, is without interest. That case is, therefore, to be excluded by the assumption that of the matrices

$$
\mathfrak{B}_{1}^{(h, l)}(\lambda) \equiv\left(\begin{array}{ll}
\mathfrak{v}_{1 h}(\lambda) & \mathfrak{b}_{1 l}(\lambda) \\
\mathfrak{v}_{2 h}(\lambda) & \mathfrak{v}_{2 l}(\lambda)
\end{array}\right), \quad h, l=1,2,3,4 ; h \neq l,
$$

at least one is not identically singular.

Let the maximum degree of the polynomials $\mathfrak{b}_{i l}(\lambda)$ be designated by $\tau$. 
The matrices (3.1) are, then, all expressible in the polynomial form

$$
\mathfrak{B}_{1}^{(h, l)}(\lambda)=\mathfrak{夭}^{(h, l, 0)}+\lambda \mathfrak{\complement}^{(h, l, 1)}+\cdots+\lambda^{\tau} \mathbb{\complement}^{(h, l, \tau)},
$$

with each symbol $(5$ standing for a constant matrix, and with

$$
\mathfrak{C}^{(h, l, \tau)} \neq \mathfrak{D},
$$

for some indices $(h, l)$. If the matrices $\left(^{(h, l, \tau)}\right.$ are not all singular, let $\tau_{2}=\tau$. Otherwise, let a set of constant elements $s_{i j}$ be determined such that the matrix $\left(s_{i j}\right)$ is nonsingular, whereas

$$
\left(s_{11}, s_{12}\right) \mathfrak{\complement}^{(h, l, \tau)} \neq \mathfrak{o},
$$

for some $(h, l)$,

$$
\left(s_{21}, s_{22}\right) \mathfrak{C}^{(h, l, \tau)}=\mathfrak{D},
$$

for all $(h, l)$. Then let $\tau_{2}$ be defined as the least integer for which

$$
\left.\left(s_{21}, s_{22}\right)\left\{\lambda^{\tau_{2}+1} \mathbb{C}^{\left(h, l, \tau_{2}+1\right)}+\cdots+\lambda^{\tau} \mathbb{C}^{(h, l, \tau)}\right)\right\} \equiv \mathbb{0},
$$

for all $(h, l)\left(^{6}\right)$. Because of the assumption made above relative to the matrices (3.1), $\tau_{2} \geqq 0$. If the boundary relation of the problem (2.3) is, then, multiplied by the matrix $\left(s_{i j}\right)$, and if thereafter the matrices $\left(s_{i j}\right) \mathfrak{B}^{(h)}(\lambda), h=0,1$, are again denoted simply by $\mathfrak{B}^{(h)}(\lambda)$, it follows that each element of these matrices is a polynomial in $\lambda$, and that when $\tau_{1}=\tau$, then $\tau_{i}$ is the maximum degree of the elements in an $i$ th row.

It may be noted now that either one of the integers $\tau_{1}$ and $\tau_{2}$ may be increased by unity by the multiplication of the boundary relation on the left by the respective matrix

$$
\left(\begin{array}{cc}
\lambda-\lambda_{1} & 0 \\
0 & 1
\end{array}\right), \quad\left(\begin{array}{cc}
1 & 0 \\
0 & \lambda-\lambda_{2}
\end{array}\right)
$$

in which $\lambda_{1}$ and $\lambda_{2}$ are any values of the parameter for which the boundary problem is initially incompatible. It is thus a matter of an adjustment of the boundary problem to assure the relations

$$
\tau_{i} \geqq 1 \text {, }
$$$$
i=1,2 \text {. }
$$

It must be noted, however, that this adjustment (which plays no role except in \$28) is not wholly formal, for since the matrix factors used in achieving it are singular for a value of $\lambda$, that value is introduced as a characteristic value by the adjustment.

Finally, in virtue of the structure of the matrices $\mathfrak{B}^{(0)}(\lambda)$ and $\mathfrak{B}^{(1)}(\lambda)$ as

( ${ }^{\circ}$ For the purposes of multiplication vectors are always to be regarded as matrices, of one row and two columns if they are left-hand factors, and of two rows and one column if they are in the role of right-hand factors. 
now arranged, it will be clear that the matrices $\mathfrak{W}^{(h)}(\lambda), h=0,1$, as defined by the formulas

$$
\begin{aligned}
& \mathfrak{W}^{(0)}(\lambda) \equiv\left(\delta_{i j} \lambda^{-\tau_{i}}\right) \mathfrak{B}^{(0)}(\lambda), \\
& \mathfrak{W}^{(1)}(\lambda) \equiv\left(\delta_{i j} \lambda^{-\tau_{i}}\right) \mathfrak{B}^{(1)}(\lambda),
\end{aligned}
$$

have elements which are polynomials in $(1 / \lambda)$, at least one element in a first row, and at least one element of a second row having a constant term which is not zero.

4. The solutions of the differential equation. Under the Hypothesis 1 of $\$ 2$, the forms and structural properties of the solutions of the matrix differential equation

$$
\mathfrak{Y}^{\prime}(x, \lambda)=\{\lambda \Re(x)+\mathfrak{Q}(x)\} \mathfrak{Y}(x, \lambda),
$$

and hence of the vector differential equation of the boundary problem (2.3) may be regarded as known $\left({ }^{7}\right)$, especially insofar as large values of the parameter are concerned. Certain of these properties are relevant to the discussion at hand and may be cited as follows.

(i) With the matrix $\mathbb{E}(x, \lambda)$ defined by the formula

$$
\mathcal{E}(x, \lambda) \equiv\left(\delta_{i j} e^{\lambda \Gamma_{i}(x)}\right),
$$

and with $\mathfrak{P}^{(0)}(x)$ defined to be identically the unit matrix, explicit formal procedures may be applied to determine successively the matrices of a sequence $\mathfrak{P}^{(h)}(x), h=1,2,3, \cdots$, so that the expression

$$
\left\{\sum_{h=0}^{\infty} \lambda^{-h \mathfrak{B}^{(h)}}(x)\right\} \Subset(x, \lambda),
$$

formally satisfies the equation (4.1), namely so that upon substitution of the expression (4.3) in the place of $\mathfrak{V}(x, \lambda)$ in the equation (4.1), the coefficients of like powers of $\lambda$ in the two resulting members of the relation are in every case equal.

(ii) The infinite series (4.3) is in general divergent. However, to each $\lambda$ half-plane of the set defined by the relations

$$
(n-1 / 2) \pi-\arg \left\{\Gamma_{1}-\Gamma_{2}\right\} \leqq \arg \lambda \leqq(n+1 / 2) \pi-\arg \left\{\Gamma_{1}-\Gamma_{2}\right\},
$$

for integral values of $n$, there corresponds an actual analytic solution of the equation (4.1) which is asymptotically represented by the expression (4.3) for the values of $\lambda$ in that half-plane.

(iii) In terms of any analytic nonsingular solution $\mathfrak{Y}(x, \lambda)$ of the equation (4.1) the general solution of that equation, and the general solution of the

(7) Cf. G. D. Birkhoff, and R. E. Langer, The boundary problems and developments associated with a system of ordinary linear differential equations of the first order. Proceedings of the American Academy of Arts and Sciences vol. 58 (1923) pp. 51-128. 
vector differential equation of the boundary problem (2.3), are given, respectively, by the formulas

$$
\mathfrak{Y}(x, \lambda) \mathfrak{C}^{(1)}, \quad \mathfrak{V}(x, \lambda) \mathfrak{c}^{(1)},
$$

in which $\mathfrak{E}^{(1)}$ and $\mathfrak{c}^{(1)}$ are an arbitrary matrix and an arbitrary vector that are independent of $x$.

If in the first of the expressions (4.5) the matrix $\mathfrak{F}^{(1)}$ is written as $\mathfrak{V}^{-1}(0, \lambda) \mathfrak{S}$, the general solution of the equation (4.1) is expressed in the form

$$
\mathfrak{V}(x, \lambda) \mathfrak{Y}^{-1}(0, \lambda) \mathfrak{C} .
$$

In this form the solution involved is wholly determined by the matrix $\mathfrak{E}$, since the form (4.6) is invariant under the substitution of any one nonsingular solution $\mathfrak{V}(x, \lambda)$ for any other one. The general solution of the vector equation (2.3) may be similarly given by the formula

$$
\mathfrak{Y}(x, \lambda) \mathfrak{Y}^{-1}(0, \lambda) \mathrm{c} .
$$

Any specific one of the solutions $\mathfrak{Y}(x, \lambda)$ to which the statement (ii) above refers, defines through the formula

$$
\mathfrak{V}(x, \lambda) \equiv \mathfrak{P}(x, \lambda) \mathfrak{E}(x, \lambda),
$$

a matrix $\mathfrak{B}(x, \lambda)$ which is analytic in $\lambda$, and which, by (ii), is such that for $\lambda$ in the respective half-plane of the set (4.4), the relation

$$
\mathfrak{P}(x, \lambda) \sim \Im+\sum_{h=1}^{\infty} \lambda^{-h \mathfrak{P}^{(h)}}(x)
$$

maintains. From this it is evident that the matrix in question is nonsingular when $|\lambda|$ is sufficiently large, and that, therefore, it may be used in the role of $\mathfrak{V}(x, \lambda)$ in the formulas (4.6) and (4.7). The asymptotic representation of the solution (4.6) or (4.7) determined by any specific matrix $\mathbb{E}$ or vector $c$ is thus obtainable from the relations (4.8) and (4.9). This representation is valid for all large values of $\lambda$, despite the fact that the matrix $\mathfrak{P}(x, \lambda)$ to which the relation (4.9) applies is different in different half-planes (4.4), precisely by virtue of the fact that the formulas (4.6), (4.7) are invariant under replacements of the solution $\mathfrak{V}(x, \lambda)$.

\section{Chapter 2}

\section{THE FAMILY OF BOUNDARY PROBLEMS}

5. The formal construction and characteristic equation of the family. Let $\kappa_{i l}, i=1,2 ; l=1,2,3,4$, be a set of constants, which for the instant may remain unspecified, and let $\nu$ be taken as a parameter whose range is to include the value zero. The formulas

$$
\begin{aligned}
& \mathfrak{B}^{(0)}(\lambda, \nu) \equiv \mathfrak{B}^{(0)}(\lambda)+\nu\left(\kappa_{i j} \lambda^{\tau_{i}}\right), \\
& \mathfrak{B}^{(1)}(\lambda, \nu) \equiv \mathfrak{B}^{(1)}(\lambda)+\nu\left(\kappa_{i, j+2} \lambda^{\tau_{i}}\right),
\end{aligned}
$$


then define the matrices which appear as their left-hand members, the elements of these matrices being polynomials in $\lambda$ and linear polynomials in $\nu$. The differential system

$$
\begin{gathered}
\mathfrak{y}^{\prime}(x, \nu)=\{\lambda \Re(x)+\mathfrak{Q}(x)\} \mathfrak{y}(x, \nu), \\
\mathfrak{B}^{(0)}(\lambda, \nu) \mathfrak{y}(0, \nu)+\mathfrak{B}^{(1)}(\lambda, \nu) \mathfrak{y}(1, \nu)=\mathfrak{b},
\end{gathered}
$$

then defines a family of boundary problems which yields the given problem (2.3) for the parameter value $\nu=0$.

If, on the pattern of the formulas (3.3), the matrices $\mathfrak{W B}^{(h)}(\lambda, \nu), h=0,1$, are now defined by the formulas

$$
\mathfrak{W}^{(h)}(\lambda, \nu)=\left(\delta_{i j} \lambda^{-r_{i}}\right) \mathfrak{B}^{(h)}(\lambda, \nu), \quad h=0,1,
$$

it follows from (3.3) and (5.1) that

$$
\begin{aligned}
& \mathfrak{W}^{(0)}(\lambda, \nu) \equiv \mathfrak{W}^{(0)}(\lambda)+\nu\left(\kappa_{i j}\right), \\
& \mathfrak{W}^{(1)}(\lambda, \nu) \equiv \mathfrak{W}^{(1)}(\lambda)+\nu\left(\kappa_{i, j+2}\right) .
\end{aligned}
$$

The elements of these matrices are therefore polynomials in $(1 / \lambda)$, of which the constant terms are linear in $\nu$ and all other terms are independent of $\nu$. Moreover, at least one element in a first row and at least one element in a second row has a constant term that does not vanish when $\nu=0$.

The general solution of the differential equation of the problem (5.2) is given by the expression (4.7), a non-trivial solution being associated with a non-vanishing vector $c$. Upon substitution of this expression into the boundary relation, the latter assumes the form

$$
\mathfrak{D}(\lambda, \nu) \mathfrak{Y}^{-1}(0, \lambda) \mathfrak{c}=\mathfrak{D},
$$

in which

$$
\mathfrak{D}(\lambda, \nu) \equiv \mathfrak{B}^{(0)}(\lambda, \nu) \mathfrak{Y}(0, \lambda)+\mathfrak{B}^{(1)}(\lambda, \nu) \mathfrak{V}(1, \lambda) .
$$

The condition that there exist a non-vanishing vector $\mathrm{c}$ to satisfy the equation (5.5), and hence that there exist a non-trivial solution (4.7) of the boundary problem is, therefore, evidently that the matrix (5.7) be singular, namely that

$$
D(\lambda, \nu)=0,
$$

where $D(\lambda, \nu)$ denotes the determinant of the matrix (5.6). The compatibility of the boundary problem corresponding to any specific value of $\nu$, is thus contingent upon $\lambda$ being a root of the characteristic equation (5.7). These roots are called the characteristic values.

It will be noted that the matrix $\mathfrak{D}(\lambda, \nu)$, and hence also its determinant $D(\lambda, \nu)$, depends upon the choice of the nonsingular solution $\mathfrak{V}(x, \lambda)$ of the equation (4.1) which appears in (5.6). However, as has already been observed, any product $\mathfrak{Y}(x, \lambda) \mathfrak{Y}^{-1}(0, \lambda)$ is independent of the solution $\mathfrak{V}(x, \lambda)$ from 
which it is formed. From this it is seen at once, that the product

$$
\mathfrak{D}(\lambda, \nu) \mathfrak{Y}^{-1}(0, \lambda)
$$

is invariant, and that the left-hand members of the equations (5.7) formed from different solutions $\mathfrak{Y}(x, \lambda)$ differ only in their non-vanishing constant factors. The characteristic values, as roots of the equation (5.7), are thus independent of the choice of $\mathfrak{Y}(x, \lambda)$.

Since the determinant $D(\lambda, \nu)$, when formed from a solution $\mathfrak{Y}(x, \lambda)$ that is analytic in $\lambda$, is itself analytic, the number of characteristic values in any bounded portion of the complex $\lambda$-plane, and hence in particular within any circle however large, is evidently finite. For the consideration of those roots which lie outside of a suitably large circle, it is convenient to construct the equation (5.7), for $\lambda$ in any half-plane of the set (4.4), from that solution to which the formulas (4.8), (4.9) apply. If the elements $a_{i l}(\lambda, \nu), i=1,2$; $l=1,2,3,4$, are defined, then, by the formulas

$$
\begin{aligned}
\left(a_{i j}\right) & \equiv \mathfrak{W}^{(0)}(\lambda, \nu) \mathfrak{B}(0, \lambda), \\
\left(a_{i, j+2}\right) & \equiv \mathfrak{W}^{(1)}(\lambda, \nu) \mathfrak{B}(1, \lambda),
\end{aligned}
$$

it is found that

$$
\mathfrak{D}(\lambda, \nu) \equiv\left(\delta_{i j} \lambda^{\tau_{i}}\right)\left(a_{i j}+a_{i, j+2} e^{\lambda \Gamma_{j}}\right) .
$$

The determinant $D(\lambda, \nu)$ is accordingly given by the formula

$$
D(\lambda, \nu) \equiv \lambda^{\tau_{1}+\tau_{2}}\left\{A_{1}-A_{2} e^{\lambda \Gamma_{1}}+A_{3} e^{\lambda\left(\Gamma_{1}+\Gamma_{2}\right)}-A_{4} e^{\lambda \Gamma_{2}}\right\},
$$

in which, if $a_{i 5}$ is interpreted as being identical with $a_{i 1}$,

$$
A_{l}(\lambda, \nu) \equiv\left|\begin{array}{cc}
a_{1 l} & a_{1, l+1} \\
a_{2 l} & a_{2, l+1}
\end{array}\right|, \quad \quad l=1,2,3,4 .
$$

Since the matrices $\mathfrak{Y}(x, \lambda)$ which enter into these formulas are different for $\lambda$ in different half-planes (4.4), the elements $a_{i l}(\lambda, \nu)$, and the determinants $A_{l}(\lambda, \nu)$ are also different functions for such different values of $\lambda$. However, each solution $\mathfrak{Y}(x, \lambda)$ in question is asymptotically described as it is used by the relation (4.9), and in this relation the matrices on the right are specific and independent of $\lambda$. It follows that each element $a_{i l}(\lambda, \nu)$, and likewise each determinant (5.12), may be taken as asymptotically equivalent to a respective formal power series in $1 / \lambda$, and thus as subject to a single representation for all large values of $\lambda$.

It is useful, for the exploitation of certain symmetries to extend the definitions of the elements $a_{i l}(\lambda, \nu)$ and of the determinants $A_{l}(\lambda, \nu)$ to all indices $l$. This may be done by the conventions

$$
\begin{array}{ll}
a_{i l_{1}} & \equiv a_{i l_{2}}, \\
A_{l_{1}} \equiv A_{l_{2}}, & \quad \text { for } l_{1} \equiv l_{2}(\bmod 4) .
\end{array}
$$


If the constants $V_{l}$ are then defined, thus, for all integers $h$

$$
V_{2 h-1}=(-1)^{h}\left\{\frac{\Gamma_{2}+\Gamma_{1}}{2}\right\}, \quad V_{2 h}=(-1)^{h}\left\{\frac{\Gamma_{2}-\Gamma_{1}}{2}\right\},
$$

it is easily verifiable that the formula (5.11) may be written in the form

$$
D(\lambda, \nu) \equiv \lambda^{\tau_{1}+\tau_{2}} e^{-\lambda V_{1}} \sum_{l=m}^{m+3}(-1)^{l+1} A_{l} e^{\lambda V_{l}},
$$

with any choice whatever of the integer $m$. The characteristic values other than zero, and hence all those which are numerically large, are thus roots of the equation

$$
A_{m} e^{\lambda V_{m}}-A_{m+1} e^{\lambda V_{m+1}}+A_{m+2} e^{\lambda V_{m+2}}-A_{m+3} e^{\lambda V_{m+8}}=0 .
$$

6. On the regularity or irregularity of a boundary problem. For any given value of $\nu$, any specific coefficient $A_{l}(\lambda, \nu)$ of the equation (5.16) is a function of $\lambda$. This function either vanishes identically or is asymptotically representable by a series in powers of $1 / \lambda$, with a constant term that may appropriately be designated by the symbol $A_{l}(\infty, \nu)$. The coefficient $A_{l}(\lambda, \nu)$ in question will be said to be regular or irregular at the given value of $\nu$, according as its constant term $A_{l}(\infty, \nu)$ is different from zero or vanishes.

If a given boundary problem is one that fulfills the hypothesis 1 in the manner of the configuration 1 of $\S 2$, the points $\lambda V_{l}$ for any four successive indices $l$, mark the vertices of a parallelogram centered at the origin in the complex $\lambda$-plane. The abscissas of these vertices evidently determine the magnitudes of the respective exponentials in the left-hand member of the characteristic equation (5.16), and that exponential which is associated with the vertex furthest to the right is the dominant one. Inasmuch as the orientation of the parallelogram is a function of $\arg \lambda$, and any specific vertex is furthest to the right for some values of $\arg \lambda$, the exponentials in the equation (5.16) may in this case all be characterized as in an obvious sense, potentially dominant.

If the boundary problem fulfills the hypothesis 1 in the manner of the configuration 2 , on the other hand, the points $\lambda V_{l}$ are collinear, and lie upon the segment terminated at $\lambda V_{1}$, and $\lambda V_{3}$ if the formulas (2.9a) apply, and on the segment terminated at $\lambda V_{2}$ and $\lambda V_{4}$ if the formulas (2.9b) are applicable. Since a position furthest to the right is impossible for all but the end points of the segment, only two of the exponentials which appear in the equation (5.16) are in this case potentially dominant.

The type of the boundary problem is essentially determined by those coefficients $A_{l}(\lambda, \nu)$ that are associated with potentially dominant exponentials in the characteristic equation. If the coefficients of the potentially dominant exponentials are all regular, the boundary problem itself is said to be of the regular type. If at least one coefficient of a potentially dominant 
exponential is irregular, but no one vanishes identically, the boundary problem is said to be of the mildly irregular type. Finally, if in the equation (5.16) at least one coefficient of a potentially dominant exponential is identically zero, but at least two coefficients of the equation are not identically zero, the boundary problem is said to be of the highly irregular type.

It will be observed at once that this classification fails to account for such boundary problems as have characteristic equations with less than two nonvanishing terms. Such problems, however, have no expansion theories associated with them. For, if in the equation (5.16) just one term is non-vanishing, the number of characteristic values is clearly finite. On the other hand, if every coefficient vanishes, the equation (5.16) is evidently vacuous. The boundary problem is then compatible for all values of $\lambda$, and no characteristic values are distinguished.

The classification thus described applies in particular to the boundary problem (2.3) which was originally given, and which is identified in the family by the parameter value $\nu=0$. The discussion at hand is concerned wholly with the case in which that problem is highly irregular. Of those coefficients $A_{l}(\lambda, 0)$ which multiply potentially dominant exponentials in the characteristic equation, at least one is therefore to be taken as identically zero. It will be supposed, primarily for the purpose of delimiting these deductions to their present bounds, that those coefficients which do not vanish identically are regular. Although this is in fact a restrictive hypothesis, inasmuch as the case in which some non-vanishing coefficients are irregular is a more general one, the features which are engendered by such irregularities are, from the standpoint here to be maintained only secondarily germane. They constitute in the first instance the salient source of the distinctions between the regular and the mildly irregular cases.

Hypothesis 2. The given boundary problem is one for which at least one coefficient of a potentially dominant exponential in the characteristic equation vanishes identically, and for which the non-vanishing coefficients are regular and at least two in number.

7. Specifications upon the family of boundary problems. Inasmuch as the constants $\kappa_{i l}$ introduced in $\$ 5$ have remained unspecified, the boundary problem of the family associated with any value of $\nu$ different from zero has been only formally defined, and its type, in particular, has remained indeterminate. This is now to be made specific. From the formulas (4.9), (5.4) and (5.9), the evaluations

$$
a_{i l}(\infty, \nu)=a_{i l}(\infty, 0)+\nu \kappa_{i l}, \quad i=1,2 ; l=1,2,3,4,
$$

are obtained. Through the relation (5.12), therefore, the expressions $A_{l}(\infty, \nu)$ are formally quadratic polynomials in $\nu$ in which the coefficients of the linear and quadratic terms are functions of the constants $\kappa_{i l}$. It is now to be stipu- 
lated that these constants be chosen so that the coefficient of each quadratic term vanishes. More precisely: The constants $\kappa_{i l}, i=1,2 ; l=1,2,3,4$, shall be such that each expression $A_{l}(\infty, \nu)$ is a linear polynomial in $\nu$, and not identically zero.

That this specification is not impossible of fulfillment in any case, may be established as follows, by the explicit display of a set of constants which have the requisite properties. Under the hypothesis 2 , there exists an index $p$ which is such that

$$
A_{p}(\lambda, 0) \equiv 0, \quad A_{p+1}(\infty, 0) \neq 0,
$$

and $A_{p+2}(\infty, 0), A_{p+3}(\infty, 0)$ are not both zero. With such an index $p$ let the constants $\kappa_{i l}$ be taken thus:

$$
\kappa_{i p}=-a_{i, p+2}(\infty, 0), \quad \kappa_{i, p+1}=0, \quad \kappa_{i, p+2}=-a_{i p}(\infty, 0), \quad \begin{array}{r}
\kappa_{i, p+3} \\
=0, \\
i=1,2 .
\end{array}
$$

It is readily computed that with these constants

$$
\begin{aligned}
A_{p}(\infty, \nu) & =\nu A_{p+1}(\infty, 0), \\
A_{p+1}(\infty, \nu) & =A_{p+1}(\infty, 0), \\
A_{p+2}(\infty, \nu) & =A_{p+2}(\infty, 0)+\nu A_{p+3}(\infty, 0), \\
A_{p+3}(\infty, \nu) & =A_{p+3}(\infty, 0)+\nu A_{p+2}(\infty, 0),
\end{aligned}
$$

and since each of these expressions is a linear polynomial in $\nu$ with at least one nonzero coefficient, they evidently all have the structure prescribed.

With the coefficients $A_{l}(\lambda, \nu)$ thus constructed, it is evidently possible to determine in the complex $\nu$-plane a closed neighborhood of the origin within which they are all regular except possibly at $\nu=0$. Such a neighborhood will be referred to as a proper region for $\nu$, and henceforth it shall be understood that all values of $\nu$ that are brought into question lie in such a region. With the parameter so delimited the family of boundary problems is now such that each of its members associated with a value of $\nu$ different from zero is of the regular type, and only the originally given problem is irregular. In an evident sense, therefore, the given highly irregular boundary problem has been imbedded in a continuous aggregate of regular problems, and appears as analytically approachable through this aggregate by the medium of a passage of the parameter to the limiting value zero. The continuing discussion is almost exclusively concerned with considerations centering upon such an approach. Inasmuch as it is adequate to the ends sought to restrict the considerations to modes of approach in which $\arg \nu$ is bounded, that restriction is to be understood henceforward.

8. Two sub-categories of highly irregular boundary problems. Rouché's theorem. The method by which a theory for boundary problems of the highly irregular type is thus to be deduced, depends essentially upon the establish- 
ment of a one to one correspondence between the characteristic values and solutions of the given problem with those of the regular problems of the imbedding family, and the consequent expression of the former as limits of the latter as $\nu \rightarrow 0$. The existence of these limits as finite values is, therefore, obviously a primary requisite, and since they may or may not all exist, depending upon the individual problem at hand, a partition of the entire category of highly irregular boundary problems into sub-categories is called for. These will be distinguished by the designations A and B. Problems in which the limits in question do all exist will be allocated to the sub-category $\mathrm{A}$, and to them the theory under deduction will be applicable. All highly irregular boundary problems of the second order for which any analyses at all are at present extant belong to this sub-category. On the other hand, problems in which some of the limits fail to exist will be allocated to the subcategory $\mathrm{B}$. To them the theory will have no application, and it seems improbable that problems of this type admit of any expansion theory of a customary sort.

A familiar theorem $\left({ }^{8}\right)$, upon which many of the considerations which follow are to be based, may be stated thus:

If within and on any specific closed contour of the complex $\lambda$-plane, two functions $\phi(\lambda)$ and $\psi(\lambda)$ are each analytic, and if on this contour the relation

$$
|\psi(\lambda)|<|\phi(\lambda)|
$$

maintains, the equation

$$
\phi(\lambda)+\psi(\lambda)=0,
$$

has precisely as many roots within the contour as has the equation

$$
\phi(\lambda)=0 .
$$

For future reference it will be noted here, that due to the manner in which the parameter $\nu$ enters into the structure of the functions $A_{l}(\lambda, \nu)$, the following may be stated.

$$
\begin{aligned}
& \text { If } A_{l}(\lambda, 0) \equiv 0 \text {, then } \\
& A_{l}(\lambda, \nu) \equiv \nu\left\{\beta_{l}+\eta_{l}(\lambda, \nu)\right\}, \quad \text { with } \beta_{l} \neq 0 . \\
& \text { If } A_{l}(\lambda, 0) \not \equiv 0 \text {, then } \\
& A_{l}(\lambda, \nu) \equiv\left\{\alpha_{l}+\beta_{l} \nu+\eta_{l}(\lambda, \nu)\right\}, \quad \text { with } \alpha_{l}+\beta_{l} \neq 0 .
\end{aligned}
$$

In either case $\eta_{l}(\lambda, \nu)$ designates a function that is asymptotically representable by a series in powers of $1 / \lambda$ with a vanishing constant term, and otherwise with coefficients that are polynomials in $\nu$. It is evident, therefore, that the relation

$$
\lim _{\lambda \rightarrow \infty} \eta_{l}(\lambda, \nu)=0,
$$

(8) Rouché's theorem. Cf. E. C. Titchmarsh, The theory of functions, Oxford, 1932, p. 116. 
maintains uniformly as to $\nu$. The coefficients $\alpha_{l}$ and $\beta_{l}$ are in every case constants.

\section{Chapter 3}

\section{BOUNDARY PROBLEMS OF THE SUB-CATEGORY B}

9. Problems of the configuration 2. In the case of any boundary problem which fulfills the hypothesis 1 in the manner of the configuration 2 of $\$ 2$, the ratio of the constants $\Gamma_{1}$ and $\Gamma_{2}$ is real. It will be shown that all such problems are to be allocated to the sub-category $B$. If the case in hand is one to which the formulas (2.9a) are applicable, the constant $\gamma$ which fulfills the relation $\Gamma_{1}=\gamma \Gamma_{2}$, is positive and greater than 1 . The potentially dominant exponentials in the characteristic equation (5.16) are those in which the subscripts are odd, and at $\nu=0$ the coefficient of at least one of these is zero. Let $p$ be chosen so that this coefficient is $A_{p}(\lambda, \nu)$. It is found then, that after division by the leading exponential the equation (5.16) may be written in the form

$$
A_{p}(\lambda, \nu)-A_{p+s}(\lambda, \nu) e^{\gamma \lambda \Gamma}+A_{p+28}(\lambda, \nu) e^{(1+\gamma) \lambda \Gamma}-A_{p+3 s}(\lambda, \nu) e^{\lambda \Gamma}=0,
$$

with $s=1$, and with $\Gamma=\Gamma_{2}$ or $\Gamma=-\Gamma_{2}$ according as $p=1$, or $p=3$. If, alternatively, the problem given is one to which the formulas (2.9b) apply the value of $\gamma$ which fulfills the relation $\Gamma_{1}=-\gamma \Gamma_{2}$ is positive and at least equal to 1 . In this case the potentially dominant exponentials are those with even subscripts, and if $A_{p}(\lambda, \nu)$ is taken as the coefficient of such a one and as vanishing at $\nu=0$, it is found that the equation (5.16) is again expressible in the form (9.1), in this instance with $s=-1$, and with $\Gamma=\Gamma_{2}$ or $\Gamma=-\Gamma_{2}$ according as $p=2$, or $p=4$. The problems of the configuration 2 may, therefore, all be analyzed by a consideration of the equation (9.1).

The case in which $\gamma=1$ may be readily disposed of. The equation (9.1) is then quadratic in $e^{\lambda \Gamma}$, and as $A_{p}(\lambda, \nu)$ tends to the limit zero with $\nu$ some roots $e^{\lambda \Gamma}$ and hence some characteristic values $\lambda$, become infinite. In the further considerations, in which it may now be assumed that $\gamma>1$, it is convenient to analyze separately the cases in which $A_{p+3 s}(\lambda, \nu)$ does not vanish with $\nu$, and that in which it does.

If the formulas (8.5) apply when $l=p+3 s$, then since (8.4) applies when $l=p$ the characteristic equation (9.1) is expressible in the form (8.2) with

$$
\begin{aligned}
& \phi=\beta_{p}-\frac{\alpha_{p+3 s}}{\nu} e^{\lambda \Gamma} \\
& \psi=\eta_{p}(\lambda, \nu)-(1 / \nu) e^{\lambda \Gamma}\left\{\beta_{p+3 s} \nu+\eta_{p+3 s}(\lambda, \nu)+A_{p+s}(\lambda, \nu) e^{(\gamma-1) \lambda \Gamma-A_{p+2 s}(\lambda, \nu) e \gamma \lambda \Gamma} .\right.
\end{aligned}
$$

The roots of the equation (8.3) are located at the points $\lambda_{m}^{*}$ given for integral values of $m$ by the formula 


$$
\lambda_{m}^{*}=(1 / \Gamma)\left\{2 m \pi i+\log \left(\frac{\beta_{p} \nu}{\alpha_{p+3 s}}\right)\right\} .
$$

If $\delta$ is any positive constant such that $\delta\left|\Gamma_{1}\right|<\pi$, these roots are enclosed individually by the circles of the nonoverlapping set

$$
\lambda=\lambda_{m}^{*}+\Delta \lambda, \quad|\Delta \lambda|=\delta,
$$

and it is seen at once that on any such circle

$$
(1 / \nu) e^{\lambda \Gamma}=\left(\beta_{p} / \alpha_{p+3 \varepsilon}\right) e^{\Gamma \Delta \lambda},
$$

and that as $\nu \rightarrow 0$,

$$
\lambda \rightarrow \infty, \text { and } e r \lambda r \rightarrow 0 .
$$

Since by the first of these relations

$$
\phi(\lambda)=\beta_{p}\left(1-e^{\Gamma \Delta \lambda}\right),
$$

on any circle (9.3), there clearly exists a positive constant $M$ which is independent of $\nu$, and such that for $\lambda$ on the circles the relation $|\phi(\lambda)|>M$ maintains. But it is also clear from the evaluations given, that $|\psi(\lambda, \nu)|<M$ whenever $|\nu|$ is sufficiently small. For all such values of $\nu$, therefore, the condition. (8.1) is fulfilled, and it follows that each circle contains a root of the equation (8.2), namely contains a characteristic value. It is evident from (9.2), however, that each of the circles (9.3) recedes to infinity as $\nu \rightarrow 0$. The enclosed characteristic values therefore approach no finite limits.

If with $l=p+3 s$ the formulas (8.4) apply, then since they also apply with $l=p$, it follows under the hypothesis 2 that the function $A_{p+s}(\lambda, \nu)$ is given by the formula (8.5). In this case the characteristic equation (9.1) may be written in the form (8.2) with

$$
\begin{aligned}
\phi= & \beta_{p}-\left(\alpha_{p+s} / \nu\right) e^{\lambda \lambda \Gamma}, \\
\psi= & \eta_{p}(\lambda, \nu)-(1 / \nu) \operatorname{er\lambda } r\left\{\beta_{p+s} \nu+\eta_{p+\varepsilon}(\lambda, \nu)-A_{p+2 s}(\lambda, \nu) e^{\lambda \Gamma}\right\} \\
& -e^{\lambda \Gamma}\left\{\beta_{p+8 s}+\eta_{p+3 \varepsilon}(\lambda, \nu)\right\} .
\end{aligned}
$$

The roots of the equation (8.3) are now located at the points

$$
\lambda_{m}^{*}=(1 / \gamma \Gamma)\left\{2 m \pi i+\log \left(\frac{\beta_{p} \nu}{\alpha_{p+\varepsilon}}\right)\right\},
$$

and with this interpretation of $\lambda_{m}^{*}$ these roots are again enclosed in the circles (9.3). On these circles it is seen that

$$
\begin{aligned}
(1 / \nu) e^{\gamma \lambda \Gamma} & =\left(\beta_{p} / \alpha_{p+s}\right) \operatorname{er} \Gamma \Delta \lambda \\
\phi(\lambda) & =\beta_{p}(1-e \gamma \Gamma \Delta \lambda)
\end{aligned}
$$

and that as $\nu \rightarrow 0$ 


$$
\lambda \rightarrow \infty, \text { and } e^{\lambda \Gamma} \rightarrow 0 .
$$

By precisely the reasoning of the previous case it is seen that each circle encloses a characteristic value and carries it to infinity as $\nu \rightarrow 0$.

The assertion that all boundary problems which conform to the configuration 2 are of the sub-category $B$, has thus been substantiated.

10. Problems for which two consecutive coefficients of the characteristic equation vanish. Since by virtue of the results of the preceding section, the continuing discussion is concerned only with boundary problems which conform to the configuration 1 , the points $V_{l}, l=1,2,3,4$, in the complex plane mark the vertices of an actual parallelogram, and each exponential in the characteristic equation is potentially dominant. Let the interior angle of this parallelogram at the vertex $V_{l}$ be designated by $\omega_{l}$. The cases upon which the attention is to be focused in this section, are those in which for some index $l$ the two consecutive coefficients, $A_{l}(\lambda, \nu)$ and $A_{l+1}(\lambda, \nu)$ vanish with $\nu$. Since the respective angles $\omega_{l}$ and $\omega_{l+1}$ are adjacent angles of the parallelogram, one of them at least does not exceed a right angle, and if this one is designated by $\omega_{p}$, the index $p$ is thereby fixed to be such that

$$
A_{p}(\lambda, 0) \equiv 0, \quad \omega_{p} \leqq \pi / 2,
$$

and also such that with either $s=1$ or $s=-1$, as the case may be,

$$
A_{p_{+} \boldsymbol{s}}(\lambda, 0) \equiv 0 \text {. }
$$

With this determination of $p$ the formulas (8.4) apply when $l=p, p+s$, and the formulas (8.5) do so when $l=p+2 s, p-s$. After division by $\nu$ and by the leading exponential, the characteristic equation (5.16) is accordingly expressible in the form (8.2) with

$$
\begin{aligned}
\phi= & \beta_{p}-\left(\alpha_{p-s} / \nu\right) e^{\lambda\left(V_{p-\sigma}-V_{p}\right)}, \\
\psi= & \eta_{p}(\lambda, \nu)-\left\{\beta_{p+\varepsilon}+\eta_{p+s}(\lambda, \nu)\right\} e^{\lambda\left(V_{p+o}-V_{p}\right)} \\
& -(1 / \nu) e^{\lambda\left(V_{p-\sigma}-V_{p}\right)}\left\{\beta_{p-s} \nu+\eta_{p-s}(\lambda, \nu)-A_{p+2 s}(\lambda, \nu) e^{\lambda\left(V_{p+s}-V_{p}\right)}\right\} .
\end{aligned}
$$

In this instance the roots of the equation (8.3) lie at the points

$$
\lambda_{m}^{*}=\frac{1}{V_{p-\varepsilon}-V_{p}}\left\{2 m \pi i+\log \left(\frac{\beta_{p} \nu}{\alpha_{p-\varepsilon}}\right)\right\} .
$$

With this interpretation of $\lambda_{m}^{*}$, these roots are enclosed in the circles of the set (9.3). On these circles

$$
\frac{1}{\nu} e^{\lambda\left(V_{p-s}-V_{p}\right)}=\frac{\beta_{p}}{\alpha_{p-1}} e^{\left(V_{p-a}-V_{p}\right) \Delta \lambda}
$$

and hence

$$
\phi=\beta_{p}\left(1-e^{\left[V_{p-t}-V_{p}\right] \Delta \lambda}\right) .
$$


The quantity on the left of the relation (10.3) is thus seen to be bounded uniformly as to $\nu$ and $m$, and the existence of a constant $M$ which is independent of $\nu$ and which is such that $|\phi|>M$ for all $\lambda$ on the circles, is evident.

Consider now those circles of the set above which are associated with the values of $m$ for which $s m$ is positive. On these circles

$$
\begin{aligned}
\lambda\left[V_{p+s}-V_{p}\right]= & \frac{V_{p+s}-V_{p}}{V_{p-8}-V_{p}}\{2 m \pi i+\log |\nu|\} \\
& +\left[V_{p+s}-V_{p}\right]\left\{\Delta \lambda+\frac{1}{V_{p+s}-V_{p}} \log \left(\frac{\beta_{p} \nu}{\alpha_{p-s}|\nu|}\right)\right\} .
\end{aligned}
$$

With the evaluation

$$
\arg \left\{\frac{V_{p+s}-V_{p}}{V_{p-s}-V_{p}}\right\}=s \omega_{p},
$$

the real part of the first term on the right of the formula (10.5) is found to be

$$
-\left|\frac{V_{p+s}-V_{p}}{V_{p-s}-V_{p}}\right|\left\{2 s m \pi \sin \omega_{p}+\cos \omega_{p} \cdot \log \frac{1}{|\nu|}\right\} \text {. }
$$

This becomes negatively infinite as $\nu \rightarrow 0$, and since the remaining term on the right of the formula (10.5) is bounded, it follows that

$$
e^{\lambda\left[V_{p+a}-V_{p}\right]} \underset{\nu \rightarrow 0}{\longrightarrow} 0 \text {. }
$$

Since for $\lambda$ on the circles in question $\lambda \rightarrow_{\nu \rightarrow 0} \infty$, the inequality $|\psi|<M$ maintains for all values of $\nu$ that are sufficiently small. For such $\nu$, then, each of these circles contains a characteristic value, and these values become infinite as $\nu \rightarrow 0$. Any boundary problem for which two consecutive coefficients of the characteristic equation vanish must, therefore, be allocated to the subcategory $\mathbf{B}$.

11. A third type of problem of the sub-category B. If for the given boundary problem the index $p$ is determined so that

$$
A_{p}(\lambda, 0) \equiv 0,
$$

it may be assumed in this continuing discussion that

$$
A_{p-1}(\lambda, 0) \not \equiv 0, \quad A_{p+1}(\lambda, 0) \not \equiv 0,
$$

since the alternative has been disposed of in $\$ 10$. The characteristic equation (5.16), after division by $\nu$ and by the leading exponential, may, therefore, be written in the form (8.2), in which the function $\phi$ is as given by the formula (10.1). The corresponding function $\psi$ is then expressible in the form

$$
\begin{aligned}
\psi=\eta_{p}(\lambda, \nu)-(1 / \nu) e^{\lambda\left(V_{p-s}-V_{p}\right)}\left\{\beta_{p-s} \nu+\eta_{p-8}(\lambda, \nu)\right. \\
\left.-\left[A_{p+2 s}(\lambda, \nu) e^{\lambda\left(V_{p-s}-V_{p}\right)}-A_{p+s}(\lambda, \nu)\right] e^{\lambda\left(V_{p+s}-V_{p-s}\right)}\right\},
\end{aligned}
$$


and in these formulas the index $s$ may be taken to be either 1 or -1 . As in the preceding section, the roots of the equation (8.3) are given by the formulas (10.2), and are thus enclosed in the respective circles (9.3). Let the attention be directed upon those circles of this set that are associated with indices $m$ for which $s m$ exceeds a certain positive value to be further determined below, and let $\lambda$ be considered upon these circles. The evaluations (10.3) and (10.4) then maintain, and for some positive constant $M$ which is independent of $\nu$, the function $\phi$ fulfills the relation $|\phi|>M$.

From the formulas (9.3) and (10.3) the equality

$$
\begin{aligned}
\lambda\left[V_{p+s}-V_{p-s}\right]= & {\left[\frac{V_{p+s}-V_{p}}{V_{p-8}-V_{p}}-1\right]\{2 m \pi i+\log |\nu|\} } \\
& +\left[V_{p+s}-V_{p-s}\right]\left\{\Delta \lambda+\frac{1}{V_{p+s}-V_{p}} \log \left(\frac{\beta_{p} \nu}{\alpha_{p-s}|\nu|}\right)\right\},
\end{aligned}
$$

may be verified. In the right-hand member of this the second term is independent of $m$ and is bounded as to $\nu$, whereas the first term has a real part which may be computed, with the use of (10.6), to be

$$
\begin{aligned}
& \frac{-1}{\left|V_{p-s}-V_{p}\right|}\left\{2 \operatorname{sm\pi }\left|V_{p+s}-V_{p}\right| \sin \omega_{p}\right. \\
& \left.\quad+\left[\left|V_{p-s}-V_{p}\right|-\left|V_{p+s}-V_{p}\right| \cos \omega_{p}\right] \log |\nu|\right\} .
\end{aligned}
$$

If the constants $V_{l}$ and $\omega_{p}$ involved in this are such that

$$
\left|V_{p-s}-V_{p}\right|-\left|V_{p+s}-V_{p}\right| \cos \omega_{p}>0, \quad s=1,-1,
$$

it is clear that the value (11.4) becomes infinite as $\nu \rightarrow 0$. The cases contrary to this are those which are here to be specifically considered.

If the boundary problem under discussion is one for which the relation (11.5) is not fulfilled, either when $s=1$ or when $s=-1$, then for such $s$ the quantity within the brace in (11.4) is arbitrarily large when $s m$ is sufficiently large, and the absolute value of the exponential

$$
e^{\lambda\left(V_{p+s}-V_{p-s}\right)}
$$

is accordingly arbitrarily small uniformly in $\nu$. It may be seen, therefore, from (11.3) and (10.3) that for all values of $\nu$ such that $|\nu|$ is suitably small and for the values of $m$ such that $s m$ exceeds a value appropriately large, the relation

$$
|\psi|<M
$$

is fulfilled. With the condition (8.1) thus met, each of the circles in question contains a characteristic value, and retains it in its interior as $\nu \rightarrow 0$. Inasmuch as the circles recede to infinity as $\nu \rightarrow 0$, it is clear that any boundary problem 
which does not satisfy both of the relations (11.5) must be allocated to the sub-category B.

The failure of either one of the relations (11.5) admits of a simple geometrical interpretation. Relative to the parallelogram with vertices at the points $V_{l}, l=1,2,3,4$, in the complex plane, the symbols $\omega_{p},\left|V_{p-8}-V_{p}\right|$, $\left|V_{p+s}-V_{p}\right|$, respectively designate the angle at the vertex $V_{p}$ and the lengths of the adjacent sides. If $\omega_{p}$ is a right angle or an obtuse angle, no failure of the condition (11.5) is possible. However, if $\omega_{p}$ is acute a failure is possible and is articulate of the fact that one of the sides of the parallelogram adjacent to the vertex $V_{p}$ is exceeded in length by the projection of the other one upon it. It will be seen at once that in such a case the diagonal $V_{p-1} V_{p+1}$ divides the parallelogram into two triangles each of which has at one of the vertices $V_{p-1}, V_{p+1}$ an angle that is not acute. The boundary problems associated with such a configuration are, therefore, those which are allocated in this section to the sub-category $B$.

As has been remarked above, the theory under deduction will have no application to boundary problems assignable to the sub-category $\mathrm{B}$. A hypothesis to disbar such problems from further consideration is, therefore, called for. To facilitate its enunciation, among other things, it is convenient to adopt here the relations

$$
\Gamma_{2 h-1}=(-1)^{h+1} \Gamma_{1}, \quad \Gamma_{2 h}=(-1)^{h+1} \Gamma_{2}, \quad h=0, \pm 1, \pm 2, \cdots,
$$

which extend the definitions of $\Gamma_{l}$ to all indices $l$. It will be noted that under them

$$
\Gamma_{l_{1}}=\Gamma_{l_{2}}, \quad \text { if } \quad l_{1} \equiv l_{2}(\bmod 4),
$$

and from the relations (5.14) that

$$
\Gamma_{l}=V_{l+1}-V_{l} .
$$

The vector $\Gamma_{l}$ thus represents the $l$ th side of the parallelogram with vertices at the points $V_{l}$, and for all $l$

$$
\arg \Gamma_{l}-\arg \Gamma_{l+1}=\omega_{l} .
$$

The characteristic equation may accordingly be written, with any choice of $l$, in the form

$$
A_{l}(\lambda, \nu)-A_{l+1}(\lambda, \nu) e^{\lambda \Gamma_{l}}+A_{l+2}(\lambda, \nu) e^{\lambda\left(\Gamma_{l}+\Gamma_{l+1}\right)}-A_{l+3}(\lambda, \nu) e^{\lambda \Gamma_{l+1}}=0 .
$$

Since the values $\left|V_{p-1}-V_{p}\right|,\left|V_{p+1}-V_{p}\right|$, are now symbolizable by $\left|\Gamma_{p-1}\right|,\left|\Gamma_{p}\right|$, and hence have in some order the values $\left|\Gamma_{1}\right|,\left|\Gamma_{2}\right|$ the conditions (11.5) may evidently be expressed in the form (iii) below.

Hypothesis 3. The given boundary problem is one whose characteristic equation, when written in the form (11.9) with $\nu=0$, fulfills the specifications:

(i) the ratio $\Gamma_{1} / \Gamma_{2}$ is not real; and 
(ii) if $A_{p}(\lambda, 0) \equiv 0$ then $A_{p-1}(\infty, 0) \cdot A_{p+1}(\infty, 0) \neq 0$, and

(iii) $\left|\Gamma_{1}\right| \cos \omega_{p}<\left|\Gamma_{2}\right|,\left|\Gamma_{2}\right| \cos \omega_{p}<\left|\Gamma_{1}\right|$.

\section{Chapter 4}

The Characteristic VAlues

12. The characteristic values for restricted values of $\nu$. In the complex $\lambda$-plane the relations

$$
\begin{aligned}
-\pi / 2-\arg \left(\Gamma_{l}-\Gamma_{l+1}\right) & \leqq \arg \lambda<-\pi / 2-\arg \left(\Gamma_{l}+\Gamma_{l+1}\right), \\
|\lambda| & \geqq N,
\end{aligned}
$$

define, for each index $l$ and for any non-negative real constant $N$, a region which is to be denoted by $S_{l}(N)$. Any four consecutive regions of this set cover the part of the plane which lies outside of the circle with radius $N$ centered at the origin. The asymptotic distribution of the characteristic values may therefore be determined by a study of their distribution in the region (12.1) with the index $l$ unspecified.

The characteristic equation of the family of boundary problems has been written in the form (11.9). If the abbreviations

$$
c_{l}(\nu) \equiv A_{l}(\infty, \nu)
$$

are adopted, the further relations

$$
A_{l}(\lambda, \nu) \equiv c_{l}(\nu)\left\{1+\chi_{l}(\lambda, \nu)\right\}
$$

define the functions $\chi_{l}(\lambda, \nu)$ here involved, and these are evidently all arbitrarily small, uniformly as to $\nu$ in any proper $\nu$-region, when $\lambda$ lies in a region (12.1) in which $N$ is sufficiently large.

Under the relation (2.8), which maintains in all boundary problems now under consideration, the constants of the set

$$
\sin \arg \left\{\frac{\Gamma_{l} \pm \Gamma_{l+1}}{\Gamma_{l+1}}\right\}, \quad l=1,2,3,4,
$$

are all positive. Let $a$ designate the smallest one of these constants, and let $a_{1}$ be any positive constant less than $a$. It is then easily verified that the relation

$$
\left|e^{\lambda \Gamma l+1}\right| \leqq e^{-a|\lambda|}
$$

for $\lambda$ in $S_{l}(N)$,

maintains, and that when $\nu$ is restricted to a part of its proper region in which

$$
|\nu| \geqq e^{-a_{1} N},
$$

then the values 


$$
\left|\frac{1}{c_{l+j}(\nu)} e^{\lambda \Gamma l+1}\right|, \quad j=0,1,
$$

are arbitrarily small in $S_{l}(N)$, if $N$ is sufficiently large.

With the symbol $r$ standing in the place of either 0 or 1 , consider the functions $\phi(\lambda, \nu)$ and $\psi(\lambda, \nu)$ defined by the formulas

in which

$$
\begin{aligned}
& \phi(\lambda, \nu) \equiv 1-\frac{c_{l+1}(\nu)}{c_{l}(\nu)} e^{\lambda \Gamma_{l}}+r\left\{\theta_{0}-\frac{c_{l+1}(\nu)}{c_{l}(\nu)} e^{\lambda \Gamma_{l} \theta_{1}}\right\}, \\
& \psi(\lambda, \nu) \equiv(1-2 r)\left\{\theta_{0}-\frac{c_{l+1}(\nu)}{c_{l}(\nu)} e^{\lambda \Gamma_{l} \theta_{1}}\right\},
\end{aligned}
$$

$$
\begin{aligned}
& \theta_{0}(\lambda, \nu) \equiv \chi_{l}(\lambda, \nu)-\frac{A_{l+3}(\lambda, \nu)}{c_{l}(\nu)} e^{\lambda \Gamma_{l+1}}, \\
& \theta_{1}(\lambda, \nu) \equiv \chi_{l+1}(\lambda, \nu)-\frac{A_{l+2}(\lambda, \nu)}{c_{l+1}(\nu)} e^{\lambda \Gamma_{l+1}}
\end{aligned}
$$

If $\lambda^{*}$ is taken to designate any zero of the function $\phi(\lambda, \nu)$ in the region $S_{l}(N)$, the equation $\phi\left(\lambda^{*}, \nu\right)=0$ may be written in the form

$$
\frac{c_{l+1}(\nu)}{c_{l}(\nu)} e^{\lambda^{*} \Gamma_{l}}=\frac{1+r \theta_{0}\left(\lambda^{*}, \nu\right)}{1+r \theta_{1}\left(\lambda^{*}, \nu\right)} .
$$

On the circle

$$
\lambda=\lambda^{*}+\Delta \lambda, \quad|\Delta \lambda|=\epsilon,
$$

in which $\epsilon$ is positive and less than the smaller of the numbers $\pi /\left|\Gamma_{j}\right|, j=1,2$, but otherwise arbitrary, the relation (12.7) yields the evaluation

$$
\frac{c_{l+1}(\nu)}{c_{l}(\nu)} e^{\lambda \Gamma_{l}}=\frac{1+r \theta_{0}\left(\lambda^{*}, \nu\right)}{1+r \theta_{1}\left(\lambda^{*}, \nu\right)} e^{\Gamma l \Delta \lambda},
$$

and since $\theta_{0}$ and $\theta_{1}$ are arbitrarily small over the region $S_{l}(N)$, it follows that on the circle (12.8) the function $\phi(\lambda, \nu)$ differs by arbitrarily little from the value $\left(1-e^{\Gamma_{l} \Delta \lambda}\right)$, while the function $\psi(\lambda, \nu)$ is arbitrarily small. Since a relation (8.1) thus maintains upon the circle (12.8), the equations (8.2) and (8.3) have the same numbers of roots within it.

Now when $r=1$ the equation (8.3) is the characteristic equation and (8.2) is the equation

$$
1-\frac{c_{l+1}(\nu)}{c_{l}(\nu)} e^{\lambda \Gamma_{l}}=0,
$$

whereas when $r=0$ the roles of these two equations are reversed. It follows 
that the roots

$$
\lambda_{l, m}^{*}=\left(1 / \Gamma_{l}\right)\left\{2 m \pi i-\log \frac{c_{l+1}(\nu)}{c_{l}(\nu)}\right\},
$$

of the equation (12.9) which lie in the region $S_{l}(N)$, may be set into one to one correspondence with the characteristic values in that region, with corresponding elements within a distance $\epsilon$ of each other. Since the points $\lambda_{l, m}^{*}$ are spaced at distances exceeding $2 \epsilon$ from each other, it must be concluded that the characteristic values in the region $S_{l}(N)$ are all simple, and that they are enumerable and denotable in the manner $\lambda_{l, m}$ so that

$$
\left|\lambda_{l, m}-\lambda_{l, m}^{*}\right|<\epsilon, \quad \text { for } \lambda_{l, m} \text { in } S_{l}(N) .
$$

Inasmuch as the constant $\epsilon$ may be taken to be arbitrarily small, and the relation (12.11) is nevertheless fulfilled when $N$ is sufficiently large, the use of the symbolism of asymptotic representation, namely

$$
\lambda_{l, m} \sim \lambda_{l, m}^{*}
$$

is evidently justified. The entire set of characteristic values is clearly enumerable, since those which lie within any circle of radius $N$ centered at the origin are finite in number, while those outside such a circle stand in correspondence with the enumerable sets (12.10) with $l=1,2,3,4$.

The relation (12.5) restricts the parameter $\nu$ from a neighborhood of the origin $\nu=0$. This prohibited neighborhood can, however, be made arbitrarily small by the choice of a value of $N$ that is sufficiently large. Irrespective of how small the proper region to which $\nu$ is initially confined may be, therefore, the considerations above are applicable for a range of values of $\nu$ that is not empty when the characteristic values concerned are remote enough from the origin of the $\lambda$-plane. Now for $\nu$ in a suitably small region, a comparison of the formulas (12.2) with (8.4) and (8.5) shows that when $\alpha_{l} \neq 0$ then the difference $\left\{\log c_{l}(\nu)-\log \alpha_{l}\right\}$ is arbitrarily small, whereas when $\alpha_{l}=0$ then $\log c_{l}(\nu)=\log \left(\beta_{l} \nu\right)$. It may be drawn from the relations (12.11), therefore, that

$$
\left|\dot{\lambda}_{l, m}-\dot{\lambda}_{l, m}^{(0)}\right|<2 \epsilon, \quad \text { for } \lambda_{l, m} \text { in } S_{l}(N),
$$

where

$$
\lambda_{k-j, m}^{(0)}=\left(1 / \Gamma_{k-j}\right)\left\{2 m \pi i-(-1)^{j} \log \frac{\alpha_{k+1-2 j}}{\beta_{k} \nu}\right\}, \quad j=0,1,
$$

when $k$ is any index for which $A_{k}(\lambda, 0) \equiv 0$, and

$$
\lambda_{q-j, m}^{(0)}=\left(1 / \Gamma_{q-i}\right)\left\{2 m \pi i-(-1)^{j} \log \frac{\alpha_{q+1-2 j}}{\alpha_{q}}\right\}, \quad j=0,1,
$$


when $q$ is an index for which $A_{q-1}(\lambda, 0) \not \equiv 0, A_{q}(\lambda, 0) \not \equiv 0$, and $A_{q+1}(\lambda, 0) \not \equiv 0$. It will be observed that the points (12.15) are constant as to $\nu$, and hence that any characteristic values represented by, them through the relation (12.13) are asymptotically constant. Under the hypotheses made, however, at most one index $q$ can exist, and there may be no such index at all for the boundary problem under consideration. In at least two and possibly in all four of the regions $S_{l}(N), l=1,2,3,4$, the characteristic values accordingly refer through the relations (12.13) to points of the respective sets (12.14), and so depend in an essential manner upon $\nu$.

It will be observed for later reference that insofar as an index $k$ is concerned to which the formulas (12.14) apply, the reasoning epitomized in the relations (12.13) would be in no way affected if the function $A_{k+2}(\lambda, \nu)$ were replaced by 0 , and the functions $A_{l}(\lambda, \nu)$ for $l=k-1, k, k+1$, were replaced by their leading terms as those are given in the formulas (8.4) and (8.5). These replacements substitute the equation

$$
\beta_{k} \nu-\alpha_{k+1} e^{\lambda \Gamma_{k}}-\alpha_{k-1} e^{\lambda \Gamma_{k+1}}=0,
$$

in the place of the characteristic equation. In the regions $S_{k-1}(N)$ and $S_{k}(N)$, therefore, the roots of this equation are also represented asymptotically by the points of the sets (12.14).

13. On critical values of $\lambda$ and $\nu$. By virtue of the hypothesis 3 the boundary problem at hand is one for which the relations (11.2) maintain if the index $p$ is suitably determined. Let such a determination of $p$ be fixed upon, and throughout this section let it be understood that $k$ is used to stand at will for either $p$ or $p+2$. For these values of $k$, the equations (12.16) are to be considered in the respective $\lambda$ half-planes $S_{k-1, k}$, of which each consists of the pair of adjacent sectors $S_{k-1}(0)$ and $S_{k}(0)$.

If, for any value of $\nu$, the equation (12.16) admits of a multiple root in the half-plane $S_{k-1, k}$, that root is a zero of the derived function

$$
\left\{\alpha_{k+1} \Gamma_{k} e^{\lambda \Gamma_{k}}+\alpha_{k-1} \Gamma_{k+1} e^{\lambda \Gamma_{k+1}}\right\} .
$$

It is, therefore, a point of the set

$$
\lambda^{(k, m)}=\frac{1}{\Gamma_{k+1}-\Gamma_{k}}\left\{(2 m+1) \pi i+\log \frac{\alpha_{k+1} \Gamma_{k}}{\alpha_{k-1} \Gamma_{k+1}}\right\}, \quad m \geqq m_{k},
$$

with the integer $m_{k}$ such that it lies in the region in question. Upon substitution of the values (13.2) into the equation (12.16), the respectively corresponding values of $\nu$ are found to be given by the formulas

$$
\nu^{(k, m)}=H_{k} e^{-m \Omega}, \quad k=p, p+2,
$$

in which each coefficient $H_{k}$ is a (complex) constant independent of $m$, whereas 


$$
\Omega=\frac{-2 \pi i \Gamma_{p}}{\Gamma_{p+1}-\Gamma_{p}}
$$

The equation (12.16) obviously defines $\nu$ as a single-valued analytic function of $\lambda$. For the deductions at hand, however, the inverse relationship, namely the dependence of $\lambda$ upon $\nu$ is of more immediate consequence. In the map defining this dependence the points (13.3) are branch points. According as the domain of $\nu$ includes these points or excludes them, the equation (12.16) may be regarded as defining $\lambda(\nu)$ as an infinitely many-valued function, or as defining its infinity of roots as distinct single-valued functions of $\nu$. Of these alternatives the latter one is to be adopted, and the points (13.2) and (13.3) are to be referred to henceforth as critical values of $\lambda$ and $\nu$, respectively. It is to be shown, among other things, that there exist in the domain of $\nu$ paths of approach to the origin which avoid the critical values, and in fact that there exist such paths along which $|\nu|$ varies monotonically and $\arg \nu$ varies within an arbitrarily prescribed positive range, and along which the roots of the equation (12.16) are uniformly bounded from the critical values of $\lambda$.

The formula (13.4), together with the hypothesis 3 , assures that both the real and the pure imaginary components of the constant $\Omega$ are positive. For $k=p$ and for $k=p+2$, therefore, the points of the respective set (13.3) lie upon a logarithmic spiral which winds in upon the point $\nu=0$, the points given by successive indices $m$ being spaced along this spiral at regular angular intervals of magnitude equal to the imaginary part of $\Omega$. Let $\kappa_{1}$ be defined as the smallest positive constant of the set

$$
\left|\frac{\Gamma_{k}}{\Gamma_{q}} e^{s \Omega}-1\right|, \quad k=p, p+2 ; q=p, p+2 ; s=0, \pm 1, \pm 2, \cdots .
$$

The relation

$$
\left|\frac{\nu^{(k, m)}-\nu^{(q, r)}}{\nu^{(q, r)}}\right| \geqq \kappa_{1},
$$

maintains then for every pair of distinct critical values $\nu^{(k, m)}$ and $\nu^{(q, r)}$, irrespective of whether they lie upon the same or different spirals.

Together with a prescription of continuity at $z=0$, the formula

$$
F(z) \equiv \frac{\Gamma_{p+1}\left(e^{\Gamma p z}-1\right)-\Gamma_{p}\left(e^{\Gamma_{p+1} z}-1\right)}{\left(\Gamma_{p+1}-\Gamma_{p}\right) z^{2}},
$$

defines $F(z)$ as a function of $z$ which is analytic over the finite $z$-plane. This function is, therefore, in particular bounded in the unit circle, and $M$ may accordingly be chosen as a constant such that $M \geqq 1$, and

$$
|F(z)|<M, \quad \text { for }|z| \leqq 1 \text {. }
$$


With any prescribed value arg $\nu_{0}$, and with any positive constant $\delta$ that is exceeded by both of the constants $\pi$ and $\kappa_{1}$, let $\sum_{\delta}$ designate the sector

$$
\left|\arg \nu-\arg \nu_{0}\right|<\delta / 2 \text {. }
$$

With such a value of $\delta$ chosen, the relations

$$
\left|\lambda-\lambda^{(k, m)}\right|<\left\{\frac{\sin (\delta / 2)}{M}\right\}^{1 / 2}, \quad k=p, p+2 ; m \geqq m_{k},
$$

define in the $\lambda$-plane a set of circular regions with fixed radii, and centered?at the critical points (13.2). Through the relation (12.16) these regions are mapped upon respective neighborhoods of the points $\nu^{(k, m)}$. These will be referred to briefly as critical neighborhoods.

From the relations (12.16) and (13.2), it may be drawn without difficulty that

$$
\frac{\nu-\nu^{(k, m)}}{\nu^{(k, m)}}=z^{2} F(z), \text { with } z=(-1)^{(k-p) / 2}\left(\lambda-\lambda^{(k, m)}\right) .
$$

It follows from this that every value $\lambda$ within a region (13.6) corresponds to a value of $\nu$ such that

$$
\left|\frac{\nu-\nu^{(k, m)}}{\nu^{(k, m)}}\right| \leqq \sin \left(\frac{\delta_{1}}{2}\right), \text { with } \delta_{1}<\delta,
$$

namely, that the critical neighborhood of the point $\nu^{(k, m)}$ is wholly within the respective circle of the set (13.7). Since $\delta_{1}<\kappa_{1}$ and $\delta_{1}<\pi$, it is clear on the one hand, because of the relation (13.5), that no two of the circles (13.7) have any points in common, and on the other hand, directly from the formula (13.7), that no one of them includes the point $\nu=0$. Since each circle furthermore subtends at $\nu=0$ the angle $\delta_{1}$, which is less than the angle of the sector $\sum_{\delta}$, the following facts are easily verified. If from the sector $\sum_{\delta}$ all points which belong to any circle of the set (13.7) are deleted, the remainder of the sector is a connected region within which there exist continuous paths of approach to the vertex $\nu=0$ along which $|\nu|$ steadily decreases. This is what was to be shown. Since along such a path $\nu$ remains in the chosen sector, the oscillation of arg $\nu$ does not exceed the prescribed value $\delta$, and since $\nu$ enters no circle (13.7), no root of the equation (12.16), either with $k=p$ or with $k=p+2$, enters into a region of the set (13.6). The roots of the equations (12.16) thus remain uniformly bounded from the critical $\lambda$-values. Paths in the $\nu$-plane having the properties enumerated will be referred to henceforth as regular paths for $\nu$.

It may be noted incidentally that the cases in which the imaginary component of the constant $\Omega / \pi$ is rational are peculiarly simple. From the formulas (13.3) it may be seen that the critical points $\nu^{(k, m)}$ then all lie upon a 
finite number of rays from the origin of the $\nu$-plane. The circles (13.7) including the critical neighborhoods are therefore centered upon these lines, and any section $\sum_{\delta}$ accordingly includes rectilinear paths of approach to $\nu=0$ that are regular.

It is familiar, and can easily be proved, that any exponential sum all of whose zeros occur at points of the set (13.2) is uniformly bounded from zero if $\lambda$ is uniformly bounded from the points in question. Any quotient obtained by the division of a function (13.1) by one of the exponentials which it involves is such a sum, and is therefore bounded from zero when $\lambda$ remains outside of the circles (13.6). With any regular path for $\nu$ there may, therefore, be associated a positive constant of $\rho$ which is such that for all values of $\nu$ upon the path the relations

$$
\begin{aligned}
\left|\alpha_{k+1} \Gamma_{k} e^{\lambda\left(\Gamma_{k}-\Gamma_{k+j}\right)}+\alpha_{k-1} \Gamma_{k+1} e^{\lambda\left(\Gamma_{k+1}-\Gamma_{k+j} \mid\right.}\right| \geqq \rho, \\
k=p, p+2 ; j=0,1,
\end{aligned}
$$

are fulfilled by every root of an equation (12.16).

14. The loci of the roots of an equation (12.16). As the parameter $\nu$ varies along any regular path, the roots of the equation (12.16) with $k=p$, in the respective half-plane $S_{p-1, p}$, remain distinct and trace out continuous loci in the $\lambda$-plane. It is to be shown that there exists for each of these loci a finite terminal point corresponding to the parameter value $\nu=0$, and hence that every root of the equation in question approaches a finite limit as $\nu \rightarrow 0$.

The change of variable and parameter from $\lambda$ and $\nu$ to $z(=x+i y)$ and $\mu$, as given by the relations

$$
\begin{gathered}
z=(i / 2)\left\{\lambda\left[\Gamma_{p}-\Gamma_{p+1}\right]+\log \frac{\alpha_{p+1}}{\alpha_{p-1}}\right\}, \\
\mu e^{i \theta}=\beta_{p} \nu \exp \left\{\frac{\Gamma_{p+1} \log \alpha_{p+1}-\Gamma_{p} \log \alpha_{p-1}}{\Gamma_{p}-\Gamma_{p+1}}\right\},
\end{gathered}
$$

with $\mu \geqq 0$, transforms the equation (12.16) into the equation

$$
e^{-z(\beta+i \alpha+i)-i \theta}+e^{-z(\beta+i \alpha-i)-i \theta}=\mu,
$$

with

$$
\alpha-i \beta=\frac{\Gamma_{p}+\Gamma_{p+1}}{\Gamma_{p}-\Gamma_{p+1}} .
$$

If $z_{0}$ indicates the point corresponding to $\lambda=0$, the region $S_{p-1, p}$ is transformed into the half-plane

$$
-\pi+\tan ^{-1} \beta / \alpha \leqq \arg \left(z-z_{0}\right) \leqq \tan ^{-1} \beta / \alpha,
$$

and this includes all except possibly a finite segment of the positive axis of reals. 
From the equality of the pure imaginary components of its two members, and the equality of their absolute values, the complex equation (14.2) may be made to yield the pair of real cartesian equations

$$
\begin{aligned}
\text { (a) } e^{y} \sin \{[1+\alpha] x+\beta y+\theta\} & =e^{-y} \sin \{[1-\alpha] x-\beta y-\theta\} \\
\text { (b) } \quad 4 e^{2 \alpha y}\left\{\cos ^{2} x+\sinh ^{2} y\right\} & =\mu^{2} e^{2 \beta x}
\end{aligned}
$$

Since $\mu$ is proportional to $|\nu|$, it may be taken to fill the role of the parameter. Along any regular path, $\theta$, which differs from $\arg \nu$ by a constant, is then determined as a function of $\mu$, the oscillation of $\theta$ being less than $\delta$ for any path in a sector $\sum_{\delta}$. By virtue of the hypothesis 3 , the real constants $\alpha, \beta$, which appear in the equations (14.5), and which are defined by the relation (14.3), are such that

$$
-1<\alpha<1, \quad 0<\beta .
$$

It may be noted, however, that in the equations (14.5) an interchange of $\alpha$ and $-\alpha$ may be achieved by the substitution of $-y$ and $-\theta$ in the place of $y$ and $\theta$. Since any result derived for $\alpha>0$ may, therefore, be translated to apply when $\alpha<0$, there is no essential loss of generality in assuming for the explicit discussion that $\alpha \geqq 0$, and this will be done in the following.

With any choice of an initial parameter value $\nu_{0}$, which is such that for the associated value $\theta_{0}$ the constant

$$
\frac{\theta_{0}+(\pi / 2) \alpha}{(1-\alpha) \pi}
$$

is not an integer, it is possible to associate an integer $n_{0}$ such that for all real constants $\delta_{0}$ which are numerically sufficiently small, the relations

$$
n_{s}+\frac{\left|\delta_{0}\right|}{\pi}<\frac{\left(q_{s}+1 / 2\right) \pi \alpha+\theta_{0}+\delta_{0} \alpha}{(1-\alpha) \pi}<\left(n_{s}+1\right)-\frac{\left|\delta_{0}\right|}{\pi},
$$

are fulfilled when $s=0$ with $q_{0}=0$. If the case is one in which $\alpha>0$, there exist then a pair of positive increasing sequences of integers $\left\{q_{8}\right\}$ and $\left\{n_{8}\right\}$, for which the relations (14.6) are fulfilled when $s=1,2,3, \cdots$. It thereupon follows further, again if $\left|\delta_{0}\right|$ is sufficiently small, that the relations

$$
n_{s}+\frac{\left|\delta_{0}\right|}{\pi}<\frac{\left(p_{s}+1 / 2\right) \pi \alpha+\theta_{0}+\delta_{0} \alpha}{(1+\alpha) \pi}<\left(n_{s}+1\right)-\frac{\left|\delta_{0}\right|}{\pi},
$$

are fulfilled by the integers of a third increasing sequence $\left\{p_{s}\right\}$. Let such sequences relative to the chosen constant $\delta_{0}$ be fixed upon. If the case is one in which $\alpha=0$, these sequences may be taken arbitrarily, since the relations (14.6), (14.7) imply no specifications for them.

Consider the relations 
(a) $y \geqq 0, \quad(1+\alpha) x+\beta y=\left(p_{s}+1 / 2\right) \pi-\theta_{0}+\delta_{0}$;

(b) $y=0, \quad x$ between $\frac{\left(p_{s}+1 / 2\right) \pi-\theta_{0}+\delta_{0}}{1+\alpha}$ and

$$
\frac{\left(q_{8}+1 / 2\right) \pi+\theta_{0}+\delta_{0}}{1-\alpha} ;
$$

(c) $y \leqq 0, \quad(1-\alpha) x-\beta y=\left(q_{s}+1 / 2\right) \pi+\theta_{0}+\delta_{0}$.

In the $(x, y)$-plane the first and third of these define half-lines which terminate upon the axis of $x$, and the second is the segment intercepted by them upon this axis. The set of relations as a whole therefore defines a broken line which divides the region (14.4) into two parts, in the one of which, to be denoted by $Z_{s}\left(\delta_{0}\right)$, the abscissas are bounded above. It is clear that a region $Z_{s}\left(\delta_{0}\right)$ with a larger index includes any one with a smaller index, and that the bound upon the abscissas increases indefinitely with $s$. Let it be assumed now that the path of $\nu$ lies in a sector $\sum_{\delta}$ for which $\delta$ fulfills the conditions imposed upon $\left|\delta_{0}\right|$ above. It is to be shown for the equations (14.5) that every root $(x, y)$ which initially lies within any region $Z_{s}(\delta)$ remains within that region as $\mu \rightarrow 0$, and that any root which is initially outside of any region $Z_{s}(-\delta)$ remains outside.

Consider any root in a position in which its ordinate is positive. For this position the equation (14.5a) shows that the sine function in the lefthand member of that equation is numerically less than unity, and hence that the value of $\{[1+\alpha] x+\beta y+\theta\}$ is not an odd multiple of $\pi / 2$. With a suitable determination of $s$, therefore,

$$
(1+\alpha) x+\beta y<\left(p_{8}+1 / 2\right) \pi-\theta_{0}+\delta,
$$

where $p_{s}$ is a member of the sequence so designated through the relation (14.7) in association with the value of $\delta_{0}=\delta$. The point $(x, y)$, therefore, lies in the region $Z_{s}(\delta)$, and since the relation (14.9) maintains while $y>0$, it is clear that the root cannot issue from this region across the boundary (14.8a). Similarly with $s$ properly redetermined and $p_{s}$ a member of the respective sequence associated with the value $\delta_{0}=-\delta$ through the relation (14.7) it is assured that

$$
\left(p_{s}+1 / 2\right) \pi-\theta_{0}-\delta<(1+\alpha) x+\beta y .
$$

The root $(x, y)$ thus lies initially outside of the region $Z_{s}(-\delta)$ with this index $s$, and since the reasoning employed above shows it to remain outside so long as $y>0$, it is evident that no root may enter any such region across the portion $(14.8 \mathrm{a})$ of its boundary.

If in any of its positions the ordinate of a root $(x, y)$ is negative, the equation (14.5a) shows that the value of $\{[1-\alpha] x-\beta y-\theta\}$ is not an odd multiple of $\pi / 2$, and hence that 


$$
\left(q_{s_{1}}+1 / 2\right) \pi+\theta_{0}-\delta<[1-\alpha] x-\beta y<\left(q_{s}+1 / 2\right) \pi+\theta_{0}+\delta,
$$

provided $s$ and $s_{1}$ are properly determined, and the sequences $\left\{q_{8}\right\}$ and $\left\{q_{s_{1}}\right\}$ are associated with the values $\delta_{0}=\delta$ and $\delta_{0}=-\delta$ respectively, through the relations (14.6). Since this configuration maintains so long as $y<0$, it follows that no root may either issue from a region $Z_{s}(\delta)$ or enter into a region $Z_{s}(-\delta)$ across a boundary $(14.8 \mathrm{c})$. form

Finally, upon setting $y=0$ the equation (14.5a) is found to reduce to the

$$
\cos x \sin (\alpha x+\theta)=0 .
$$

Of the roots of this equation those that are zeros of the factor $\cos x$ lie at points of the set

$$
[(r+1 / 2) \pi, 0], \quad r=0,1,2, \cdots .
$$

They are shown by the equation (14.5b) to be uniquely associated with the parameter value $\mu=0$, and thus, as points of loci which are traced out as $\mu \rightarrow 0$, they are terminal points, and not points at which the loci actually cross the axis of $x$. Such crossing points must accordingly be zeros of the factor $\sin (\alpha x+\theta)$, and hence points at which the respective values of $\left\{\alpha x+\theta_{0}\right\}$ differ from integral multiples of $\pi$ by less than the amount $\left|\delta_{0}\right|$. If $\alpha>0$ no such point lies on any segment $(14.8 \mathrm{~b})$, either for $\delta_{0}=\delta$ or for $\delta_{0}=-\delta$, since the relations (14.6) and (14.7) insure that on any such segment

$$
n_{s} \pi+\left|\delta_{0}\right|<\alpha x+\theta_{0}<\left(n_{s}+1\right) \pi-\left|\delta_{0}\right| \text {. }
$$

On the other hand if $\alpha=0$ there exist no such points at all, as may be seen from the relation (14.6) with $s=0$. Since a root may, therefore, neither issue from a region $Z_{s}(\delta)$ nor enter into a region $Z_{s}(-\delta)$ over the boundary $(14.8 \mathrm{~b})$, the assertion above has been substantiated.

This deduction admits of two specific and pertinent conclusions. In the first instance, since every root remains within some region $Z_{\mathbf{s}}(\delta)$, its abscissa is subject to some upper bound. By the equation (14.5b), therefore, it approaches a limit as $\mu \rightarrow 0$, and this limit is a point of the set (14.10). In the second instance, since no root may enter into any region $Z_{8}(-\delta)$, it follows that the distance of any root from the point $z_{0}$ is subject to a lower bound, and that this bound is arbitrarily large for any root which is sufficiently distant at any specific value of $\mu$.

As reinterpreted into terms of the variables $\lambda$ and $\nu$ through the formulas (14.1), the results may be formulated thus. The roots $\lambda^{\prime}$ of the equation (12.16) with $k=p$ which lie in the half-plane $S_{p-1, p}$, all approach finite limits as $\nu \rightarrow 0$, and these limits are all points of the set

$$
\frac{-1}{\Gamma_{p}-\Gamma_{p+1}}\left\{(2 r+1) \pi i+\log \frac{\alpha_{p+1}}{\alpha_{p-1}}\right\} \text {. }
$$


Any such root $\lambda^{\prime}$ which at any specific value of $\nu$ is sufficiently large in absolute value, remains arbitrarily large as $\nu \rightarrow 0$.

15. The characteristic values as $\nu \rightarrow 0$. In $\$ 12$ it was deduced that if there exists for the given boundary problem an index $q$, such that $A_{l}(\lambda, 0) \not \equiv 0$ for $l=q-1, q, q+1$, then the characteristic values which lie in the regions $S_{q-1}(N)$ and $S_{q}(N)$ are represented by the points of the sets (12.10) with $l=q-1, q$. Insofar as these deductions were concerned, the restriction (12.5) upon the parameter $\nu$ was wholly dispensable, since the relation (12.4) insures that the values (12.6) are arbitrarily small when $N$ is suitably large, without recourse to the restriction upon $\nu$. The representations of the characteristic values by the relations (12.13), (12.15) are, therefore, not only valid as stated in $\$ 12$, but maintain as $\nu \rightarrow 0$.

The contrary is the case insofar as the characteristic values are concerned, which lie in any region $S_{p-1}(N)$ or $S_{p}(N)$ with an index $p$ for which $A_{p}(\lambda, 0) \equiv 0$. That these values cannot be represented for unrestricted values of $\nu$ by the points (12.14) through the relation (12.13), is, in fact, immediately evident, since these points recede to infinity as $\nu \rightarrow 0$. For the deductions culminating in the relations (12.13), (12.14) the restriction (12.5) was, therefore, essential, and it accordingly remains to deduce for the characteristic values in any halfplane $S_{p-1, p}$ a representation which maintains as $\nu \rightarrow 0$.

For $\lambda$ in a region $S_{p}(N)$, let the function $\phi(\lambda)$ be defined by the formula

$$
\phi(\lambda) \equiv \beta_{p} \nu e^{-\lambda \Gamma_{p}}-\alpha_{p+1}-\alpha_{p-1} e^{\lambda\left(\Gamma_{p+1}-\Gamma_{p}\right)} .
$$

With $\lambda=\lambda^{\prime}+\Delta \lambda$, in which $\lambda^{\prime}$ is any root of the equation (12.16), an alternative formula for this function is

$$
\phi(\lambda)=\left\{\phi_{1}\left(\lambda^{\prime}\right)-\phi_{2}(\Delta \lambda)\right\} \phi_{3}(\Delta \lambda),
$$

in which

$$
\begin{aligned}
\phi_{1}\left(\lambda^{\prime}\right) & \equiv\left\{\alpha_{p+1} \Gamma_{p}+\alpha_{p-1} \Gamma_{p+1} e^{\lambda^{\prime}\left(\Gamma_{p+1}-\Gamma_{p}\right)}\right\}, \\
\phi_{2}(\Delta \lambda) & \equiv \alpha_{p+1}\left\{\Gamma_{p}-\Gamma_{p+1} \frac{1-e^{\Gamma_{p} \Delta \lambda}}{1-e^{\Gamma_{p+1} \Delta \lambda}}\right\} \\
\phi_{3}(\Delta \lambda) & \equiv e^{-\Gamma_{p} \Delta \lambda}\left\{\frac{1-e^{\Gamma_{p+1} \Delta \lambda}}{\Gamma_{p+1}}\right\} .
\end{aligned}
$$

If the parameter $\nu$ lies on a regular path, the relation (13.8) with $k=p$ and $j=0$, insures the existence of a positive constant $\rho$ which is such that $\left|\phi_{1}\left(\lambda^{\prime}\right)\right|>\rho$ for all choices of the root $\lambda^{\prime}$ and all positions of $\nu$ on the path. The functions $\phi_{2}(\Delta \lambda)$ and $\phi_{s}(\Delta \lambda)$ are analytic for small values of $\Delta \lambda$, and vanish at $\Delta \lambda=0$. A positive constant $\epsilon$ may be determined, therefore, such that when $|\Delta \lambda|=\epsilon$, then $\left|\phi_{2}(\Delta \lambda)\right| \leqq \rho / 2$, and $\left|\phi_{s}(\Delta \lambda)\right| \neq 0$. It is clear, then, that on the circle

$$
\lambda=\lambda^{\prime}+\Delta \lambda, \quad|\Delta \lambda|=\epsilon,
$$


the function $\phi(\lambda)$ fulfills a relation

$$
|\phi(\lambda)|>M
$$

with some positive constant $M$.

Let the function $\psi(\lambda)$ be defined by the formula

$$
\begin{aligned}
\psi(\lambda) \equiv & \left\{A_{p}(\lambda, \nu)-\beta_{p} \nu\right\} e^{-\lambda \Gamma_{p}}-\left\{A_{p+1}(\lambda, \nu)-\alpha_{p+1}\right\}+A_{p+2}(\lambda, \nu) e^{\lambda \Gamma^{\prime} p+1} \\
& \left.-\left\{A_{p+1}(\lambda, \nu)-\alpha_{p+1}\right\} e^{\lambda\left(\Gamma_{p+1}-\Gamma_{p}\right)}\right\} .
\end{aligned}
$$

On the circle (15.1) an alternative expression for $\psi(\lambda)$ is

$$
\begin{aligned}
\psi(\lambda)= & A_{p+2}(\lambda, \nu) e^{\lambda \Gamma_{p+1}}+\left\{\eta_{p} \frac{\alpha_{p+1}}{\beta_{p}} e^{-\Gamma_{p} \Delta \lambda}-\eta_{p+1}-\beta_{p+1} \nu\right\} \\
& +\left\{\eta_{p} \frac{\alpha_{p+1}}{\beta_{p}} e^{-\Gamma_{p+1} \Delta \lambda}-\eta_{p+1}-\beta_{p-1} \nu\right\} e^{\lambda\left(\Gamma_{p+1}-\Gamma_{p}\right)}
\end{aligned}
$$

and from this it may be seen that

$$
|\psi(\lambda)|<M
$$

provided $N$ is sufficiently large and $|\nu|$ suitably small, since the exponential $\exp \left\{\lambda\left(\Gamma_{p+1}-\Gamma_{p}\right)\right\}$ is bounded in the region $S_{p}(N)$, and the functions $\exp \left\{\lambda \Gamma_{p+1}\right\}$ and $\eta_{l}(\lambda, \nu)$, are then arbitrarily small.

By the deductions of $\$ 12$, both the characteristic values and the roots of the equation (12.16) in the region $S_{p}(N)$, are represented with an arbitrary degree of accuracy at any specific value of $\nu(\neq 0)$ by the set of points (12.14) with $k=p, j=0$, if the value of $N$ is sufficiently large. Each characteristic value thus corresponds to and is represented in an obvious sense by the respective root $\lambda^{\prime}$. Since with the definitions (15.1) and (15.4) the equation (8.2) is the characteristic equation, it follows from the relations (15.3) and (15.5) by reasoning which is now familiar, that the circle (15.2) contains and retains a characteristic value within it, and therefore that $\lambda^{\prime}$ continues to represent its associated characteristic value, so long as it remains in the region $S_{p}(N)$.

By the formal interchange of the symbols $\alpha_{p-1}, \beta_{p-1}$, and $\Gamma_{p}$ with $\alpha_{p+1}$, $\beta_{p+1}$, and $\Gamma_{p+1}$ respectively, the deductions given above may be adapted to the consideration of the characteristic values and roots $\lambda^{\prime}$ in the region $S_{p-1}(N)$. Since it was found in $\$ 14$, that any root $\lambda^{\prime}$ which at an initial value of $\nu$ lies in the domain comprised of the regions $S_{p-1}(N)$ and $S_{p}(N)$ remains in this domain as $\nu \rightarrow 0$, the asymptotic representability of the characteristic values in the half-plane $S_{p-1, p}$ by means of the roots of the equation (12.16) as $\nu \rightarrow 0$, has been established. In particular, therefore, every characteristic value $\lambda_{l_{m}}$ approaches a finite limit as $\nu \rightarrow 0$, and $\left|\lambda_{l m}\right|$ is subject to a lower bound, which is indefinitely large with the index $m$ and is independent of the parameter $\nu$. 
Since as $\nu \rightarrow 0$ along a regular path each circle (15.2) contains precisely one characteristic value, it is a particular consequence that all such values lying outside of some circle centered at $\lambda=0$ are simple, and that multiplicity is accordingly possible at most in the instance of members of the finite set which lies within such a circle. The following consideration shows, therefore, that multiplicities of the characteristic values may be wholly obviated for values of $\nu$ different from zero by an appropriate choice of the path of $\nu$.

Within any circle about the origin, the determinant $D(\lambda, \nu)$ given by the formula (5.15), and $D_{\lambda}(\lambda, \nu)$, its partial derivative as to $\lambda$, are analytic functions of $\lambda$ and polynomials in $\nu$. Their $\nu$ eliminant, therefore, has at most a finite number of zeros within the circle, and these zeros correspond through the characteristic equation (5.7) to the values of $\nu$ for which a multiple characteristic value is possible. Inasmuch as these values of $\nu$ are thus also finite in number they may,- - except for $\nu=0$, if that is among them,--be avoided by the choice of the path of $\nu$. It will be assumed in the following that any path of $\nu$ that is brought into question does avoid these points. The relation

$$
D_{\lambda}(\lambda, \nu) \neq 0,
$$

for $\nu \neq 0$,

is then fulfilled by every characteristic value.

\section{Chapter 5}

\section{SEquences of Contours in the $\lambda$-PLANE}

16. An ordering of the characteristic values. Through their designation in the manner $\lambda_{l, m}$ the characteristic values have been grouped into sub-sets which are distinguished by the respective index values $l=1,2,3,4$. For the continuing discussion advantages no longer subsist in this, and these values may profitably be regarded hence forth as members of a single simple sequence, in which the ordering is specifically such as will be described in the following.

Let $\delta$ be chosen as any positive constant which fulfills the relations

$$
\delta<\frac{\pi}{18\left|\Gamma_{j}\right|}, \quad j=1,2,
$$

and let $\delta_{1}$ thereupon designate the smaller one of the values

$$
(\delta / 3)\left|\Gamma_{j}\right|, \quad j=1,2 .
$$

For those indices $l$ for which $A_{l}(\lambda, 0) \equiv 0$ the functions $c_{l}(\nu)$, given by the relations (12.2), are constant multiples of $\nu$, and hence if $\nu_{0}$ is an initial parameter value (different from zero) on any regular path that lies in a sector $\sum_{\delta_{1}}$, the relation

$$
\left|\arg c_{l}\left(\nu_{0}\right)-\arg c_{l}(\nu)\right|<\delta_{1}
$$


is fulfilled along that path. For those indices $l$ for which $A_{l}(\lambda, 0) \neq 0$, on the other hand, the functions $c_{l}(\nu)$ approach non-vanishing limits as $\nu \rightarrow 0$. There exists, therefore, a neighborhood of the origin in which the oscillations of the respective functions arg $c_{l}(\nu)$ remain less than $\delta_{1}$, and hence if $\nu_{0}$ is chosen in such a neighborhood the relation (16.2) is fulfilled for all indices $l$ when $\nu$ is on the path segment terminated by $\nu_{0}$ and the origin. It will be supposed in the following that $\nu_{0}$ is so chosen, and that $\nu$ varies on such a path segment. The relations

$$
\left|\frac{1}{\Gamma_{h}} \arg \frac{c_{l}\left(\nu_{0}\right)}{c_{l}(\nu)}\right|<\frac{\delta}{3},
$$

then maintain for all indices $h$ and $l$.

Let the characteristic values be ordered now into a simple sequence

$$
\lambda_{r}(\nu), \quad r=1,2,3, \cdots,
$$

with an ordering such that at $\nu_{0}$ their absolute values stand in a non-decreasing succession, that is,

$$
\left|\lambda_{r}\left(\nu_{0}\right)\right| \leqq\left|\lambda_{r+1}\left(\nu_{0}\right)\right|, \quad r=1,2,3, \cdots .
$$

Through the asymptotic relationship (12.12), which maintains at $\nu=\nu_{0}$, this ordering evidently serves immediately to order also the corresponding points (12.10), at least insofar as those with sufficiently large indices $m$ are concerned, into the sequence

$$
\lambda_{r}^{*}(\nu), \quad r=r_{1}, r_{1}+1, r_{1}+2, \cdots .
$$

Inasmuch as each member of this latter sequence is drawn from one of the four sub-sets (12.10), it is clear that any consecutive five of them must include at least two from some one of the sub-sets. To every sufficiently large index $r$, therefore, there corresponds some index pair $(l, m)$ such that

$$
\left|\lambda_{r+4}^{*}\left(\nu_{0}\right)\right|-\left|\lambda_{r}^{*}\left(\nu_{0}\right)\right| \geqq\left|\lambda_{l, m+1}^{*}\left(\nu_{0}\right)\right|-\left|\lambda_{l, m}^{*}\left(\nu_{0}\right)\right| .
$$

In this relation the right-hand member differs by arbitrarily little from the value $2 \pi /\left|\Gamma_{l}\right|$, whenever $m$ is large enough, as may be seen from the formula (12.10). It follows in particular, from the relation (16.1), that the left-hand member of the inequality (16.7) exceeds the value $32 \delta$ whenever $r$ exceeds some specifiable value, and hence that for every such index $r$ at least one of the differences

$$
\left|\lambda_{r+1+j}^{*}\left(\nu_{0}\right)\right|-\left|\lambda_{r+j}^{*}\left(\nu_{0}\right)\right|, \quad j=0,1,2,3,
$$

exceeds $8 \delta$. It may be asserted, therefore, that there exists an increasing sequence of integers $n$ of which no one exceeds its predecessor by more than four, and for each of which the relation 


$$
\left|\lambda_{n+1}^{*}\left(\nu_{0}\right)\right|-\left|\lambda_{n}^{*}\left(\nu_{0}\right)\right|>8 \delta,
$$

is valid.

17. The sequence of contours $C_{n}$. For each index $n$ for which the relation (16.8) is fulfilled, let the circle in the $\lambda$-plane which is centered at the origin and of the radius $\Lambda_{n}$, with

$$
\Lambda_{n}=(1 / 2)\left\{\left|\lambda_{n+1}^{*}\left(\nu_{0}\right)\right|+\left|\lambda_{n}^{*}\left(\nu_{0}\right)\right|\right\},
$$

be designated as the contour $C_{n}$. It will be seen at once that

$$
\begin{array}{ll}
\left|\lambda_{r}^{*}\left(\nu_{0}\right)\right|<\Lambda_{n}-4 \delta, & \text { for } r \leqq n, \\
\left|\lambda_{r}^{*}\left(\nu_{0}\right)\right|>\Lambda_{n}+4 \delta, & \text { for } r>n,
\end{array}
$$

and hence that at $\nu=\nu_{0}$ no point of the sequence (16.6) lies within a distance of $4 \delta$ from any one of the contours $C_{n}$. It is to be shown that a succession of points $\nu_{n}$ may be chosen on the path $\nu$, such that $\left|\nu_{n}\right|$ decreases monotonically to zero, and such that no characteristic value lies within a distance of $\delta$ from the contour $C_{n}$ when $\nu$ is between $\nu_{0}$ and $\nu_{n}$, namely when $\nu$ is on the "path segment" $\left(\nu_{0}, \nu_{n}\right)$.

If $x, y, x_{0}$, and $y_{0}$ are any real values, and $z=x+i y, z_{0}=x_{0}+i y_{0}$ the relation

$$
\left|z^{2}\right|-\left|z_{0}^{2}\right|=\left(x^{2}-x_{0}^{2}\right)+\left(y-y_{0}\right)\left(y+y_{0}\right),
$$

is an obvious one, which leads easily to the inequality

$$
|| z|-| z_{0}|| \leqq \frac{\left|x^{2}-x_{0}^{2}\right|}{|z|+\left|z_{0}\right|}+\left|y-y_{0}\right| .
$$

Let any member of the set (16.6) be chosen, and let the indices $(l, m)$ be determined so that this member is also given by the formula (12.10). Then with

$$
\begin{aligned}
& x=\frac{1}{\left|\Gamma_{l}\right|} \log \left|\frac{c_{l}(\nu)}{c_{l+1}(\nu)}\right|, \\
& y=\frac{1}{\left|\Gamma_{l}\right|}\left\{2 m \pi i+\arg \frac{c_{l}(\nu)}{c_{l+1}(\nu)}\right\},
\end{aligned}
$$

and $x_{0}=x\left(\nu_{0}\right), y_{0}=y\left(\nu_{0}\right)$, the formula (12.10) yields $|z|=\left|\lambda_{r}^{*}(\nu)\right|,\left|z_{0}\right|$ $=\left|\lambda_{r}^{*}\left(\nu_{0}\right)\right|$.

At $\nu=\nu_{0}$, the relations

$$
\left\{\frac{1}{\left|\Gamma_{l}\right|} \log \left|\frac{c_{l}(\nu)}{c_{l+1}(\nu)}\right|\right\}^{2}<(\delta / 3) \Lambda_{n}, \quad l=1,2,3,4,
$$

are all fulfilled for every sufficiently large index $n$. They evidently continue to be fulfilled as $\nu$ varies from $\nu_{0}$, so long as it remains subject to a condition 


$$
\log \left|\frac{1}{\nu}\right| \leqq a_{2} \Lambda_{n}^{1 / 2}
$$

in which $a_{2}$ is an appropriately determined positive constant. With each index $n$ concerned there may, therefore, be associated a point $\nu_{n}$ on the path of $\nu$ for which the equality in the relation (17.6) applies. The condition (17.5) is then clearly fulfilled when $\nu$ is between $\nu_{0}$ and $\nu_{n}$, whereas the sequence $\left|\nu_{n}\right|$ converges monotonically to zero, as was asserted above. With $\nu$ on the path segment $\left(\nu_{0}, \nu_{n}\right)$, the formulas (17.5) and (16.3) show at once that

$$
\left|x^{2}-x_{0}^{2}\right|<(\delta / 3) \Lambda_{n}
$$

and

$$
\left|y-y_{0}\right|<(2 \delta / 3) \text {. }
$$

The relation (17.3) accordingly yields the inequality

$$
|| \lambda_{r}^{*}(\nu)|-| \lambda_{r}^{*}\left(\nu_{0}\right)||<\frac{(1 / 3) \delta \Lambda_{n}}{\left|\lambda_{r}^{*}(\nu)\right|+\left|\lambda_{r}^{*}\left(\nu_{0}\right)\right|}+2 \delta / 3,
$$

and from this, together with the relations (17.2), it may be concluded that

$$
\begin{array}{ll}
\left|\lambda_{r}^{*}(\nu)\right|<\Lambda_{n}-2 \delta, & \text { for } r \leqq n, \\
\left|\lambda_{r}^{*}(\nu)\right|>\Lambda_{n}+2 \delta, & \text { for } r>n .
\end{array}
$$

By the deductions of $\$ 12$ the relations (12.11), with any positive $\epsilon$, are fulfilled for all sufficiently large values of $N$, and maintain while $\nu$ fulfills the respective condition (12.5). Since in these deductions the role of $\epsilon$ may be taken by the constant $\delta$ above, and since the corresponding role of $N$ is then filled by any of the constants $\Lambda_{n}-2 \delta$ in which $n$ is sufficiently large, it follows that all characteristic values $\lambda_{r}(\nu)$ which lie outside of the circle $|\lambda|=\Lambda_{n}-2 \delta$, fulfill a relation

$$
\left|\lambda_{r}(\nu)-\lambda_{r}^{*}(\nu)\right|<\delta,
$$

and do so for all values of $\nu$ that satisfy the condition

$$
|\nu| \geqq e^{-a_{1}\left(\Lambda_{n}-2 \delta\right)} \text {. }
$$

Inasmuch as the relation

$$
a_{1}\left(\Lambda_{n}-2 \delta\right)>a_{2} \Lambda_{n}^{1 / 2},
$$

is fulfilled for all sufficiently large indices $n$, this specification upon $\nu$ is implied by the condition (17.6). The relation (17.9) thus applies in particular over the path segment $\left(\nu_{0}, \nu_{n}\right)$, and from this, together with the inequalities (17.8), it follows at once that on this path segment the relations 


$$
\begin{aligned}
& \left|\lambda_{r}(\nu)\right|<\Lambda_{n}-\delta, \\
& \left|\lambda_{r}(\nu)\right|>\Lambda_{n}+\delta,
\end{aligned}
$$

for $r \leqq n$,

for $r>n$,

maintain.

No characteristic value comes within a distance of $\delta$ of any contour $C_{n}$ associated with a sufficiently large index $n$, therefore, while $\nu$ varies along its path from $\nu_{0}$ to $\nu_{n}$.

18. The determinant $D(\lambda, \nu)$ on the contour $C_{n}$. As it is expressed through the formula (5.15), the determinant $D(\lambda, \nu)$ is the sum of four terms with coefficients $A_{l}(\lambda, \nu)$ that are of the structure (12.2), (12.3). It is to be shown that when, with a sufficiently large index $n, \lambda$ and $\nu$ are respectively on the contour $C_{n}$, and the path segment $\left(\nu_{0}, \nu_{n}\right)$, the functions $B_{l}(\lambda, \nu)$ defined by the formulas

$$
B_{l}(\lambda, \nu) \equiv \frac{1}{D(\lambda, \nu)}\left\{(-1)^{l+1} \lambda^{r_{1}+\tau_{2}} c_{l}(\nu) e^{\lambda\left(V_{l}-V_{1}\right)}\right\},
$$

are bounded uniformly as to $n$, namely that there exists some constant $M$, independent of $n$ and $l$, such that for all indices $l$

$$
\left|B_{l}(\lambda, \nu)\right|<M, \text { for } \lambda \text { on } C_{n} \text {, and } \nu \text { on }\left(\nu_{0}, \nu_{n}\right) \text {. }
$$

When $|\lambda|=\Lambda_{n}$, with a sufficiently large index $n$, and $\nu$ is on $\left(\nu_{0}, \nu_{n}\right)$, the values

$$
\left|c_{l}(\nu) e^{\lambda V l}\right|, \quad l=1,2,3,4,
$$

all differ from zero. As $\lambda$ traces the circle $C_{n}$, each of these values is in its turn the dominant one upon a respective arc of the circle. If this arc upon which the largest of the values (18.3) is that given by the index $h$, is denoted by $C_{n}^{(h)}$, the relations

$$
\left|\frac{c_{l}(\nu) e^{\lambda V l}}{c_{h}(\nu) e^{\lambda V_{h}}}\right| \leqq 1, \quad \quad \text { for } \lambda, \nu \text {, on } C_{n}^{(h)} \text { and }\left(\nu_{0}, \nu_{n}\right),
$$

are fulfilled for all $l$, and it is accordingly clear that on this arc the index $h$ also marks the dominant one of the functions (18.1). The relations (18.2) will, therefore, be established if it is shown that there exists a constant $M$ such that for every $h$

$$
\left|B_{h}(\lambda, \nu)\right|<M, \text { for } \nu \text { on }\left(\nu_{0}, \nu_{n}\right) \text { and } \lambda \text { on } C_{n}^{(h)} \text {. }
$$

From the relations (12.1), the arc $C_{n}^{(h)}$ is seen to lie partly in each of the sectors $S_{h}$ and $S_{h-1}$. It consists, therefore, of two contiguous arcs which may be conveniently denoted by $C_{n}^{(h, j)}, j=0,1$, and which lie in the respective regions $S_{h-j}$. On each of these arcs the inequality (18.5) may be established in the manner of the following. The formulas (18.1), (5.15) and (12.3) yield for the reciprocal of $B_{h}(\lambda, \nu)$ the expression 


$$
\begin{aligned}
\frac{1}{B_{h}(\lambda, \nu)}=1+\chi_{h} & -\frac{A_{h+1}}{c_{h}} e^{\lambda\left(V_{h+1}-V h\right)}+\frac{A_{h+2}}{c_{h}} e^{\lambda\left(V_{h+2}-V h\right)} \\
& -\frac{A_{h+3}}{c_{h}} e^{\lambda\left(V_{h+\gamma}-V_{h}\right)} .
\end{aligned}
$$

By the use of the relations (11.7) the final two terms in this may be written in the form

$$
A_{h+2}\left\{\frac{c_{h+1} e^{\lambda V_{h+1}}}{c_{h} e^{\lambda V_{h}}}\right\}\left\{\frac{1}{c_{h+1}} e^{\lambda \Gamma_{h+1}}\right\}-A_{h+3}\left\{\frac{1}{c_{h}} e^{\lambda \Gamma_{h+1}}\right\} .
$$

On the arc $C_{n}^{(h, 0)}$, therefore, their sum is arbitrarily small, in virtue of the relation (18.4), and the fact that with $\lambda$ in the domain $S_{l}\left(\Lambda_{n}\right)$ and $\nu$ on $\left(\nu_{0}, \nu_{n}\right)$ the values (12.6) are arbitrarily small. The remaining terms on the right of the relation (18.6) are expressible in the manner

$$
\left\{1-\frac{c_{h+1}}{c_{h}} e^{\lambda \Gamma_{h}}\right\}+\left\{\chi_{h}-\chi_{h+1} \frac{c_{h+1} e^{\lambda V_{h+1}}}{c_{h} e^{\lambda V_{h}}}\right\} \text {. }
$$

In this the first member is identical with the function

$$
\left\{1-e^{\left(\lambda-\lambda_{h, n}^{*}\right) \Gamma_{h}}\right\}
$$

because of the formula (12.10), whereas the remaining member is again arbitrarily small in virtue of the relation (18.4) and the fact that the functions $\chi_{l}(\lambda, \nu)$ approach zero uniformly as $|\lambda| \rightarrow \infty$. Since with $\nu$ on the path segment $\left(\nu_{0}, \nu_{n}\right)$ and $\lambda$ on the arc $C_{n}^{\left(h_{0}\right)}$, the value $\left(\lambda-\lambda_{h, n}^{*}\right) \Gamma_{h}$ is bounded from the multiples of $2 \pi i$, uniformly as to $n$, as was shown in $\$ 17$, it follows that the function (18.7), and hence the entire right-hand member of the formula (18.6), is uniformly bounded from zero. Thus with a suitable constant $M$, the relation (18.5) is established insofar as the values of $\lambda$ on the $\operatorname{arcs} C_{n}^{(m, 0)}$ are concerned.

For the discussion relative to the arcs $C_{n}^{(h, 1)}$ the reasoning above may be essentially adapted by the mere interchange of the roles of the third and fifth terms on the right of the formula (18.6). Thus the sum of the third and fourth terms, when written in the form

$$
-A_{h+1}\left\{\frac{1}{c_{h}} e^{\lambda \Gamma h}\right\}+A_{h+2}\left\{\frac{c_{h-1} e^{\lambda V_{h-1}}}{c_{h} e^{\lambda V_{h}}}\right\}\left\{\frac{1}{c_{h-1}} e^{\lambda \Gamma h}\right\},
$$

is seen to be arbitrarily small, since that is true of the expressions (12.6) with $l=h-1$. The remaining terms on the right of the formula (18.6) are expressible in the form

$$
\left\{1-e^{-\left(\lambda-\lambda_{h-1, n}^{*}\right)_{h-1}}\right\}+\left\{\chi_{h}-\chi_{h-1} \frac{c_{h-1} e^{\lambda V_{h-1}}}{c_{h} e^{\lambda V_{h}}}\right\}
$$


and this is uniformly bounded from zero, as was the value (18.7). The existence of a constant $M$ for which the inequalities (18.5), and hence (18.2), are valid, may thus be regarded as established.

\section{Chapter 6}

\section{ADJOINT BOUNDARY PROBLEMS}

19. The definition and the solutions of adjoint boundary problems. In terms of the square matrices which appear as coefficients in the equations (5.2), the set of relations

$$
\begin{aligned}
\mathfrak{z}^{\prime}(x, \nu) & =-\mathfrak{z}(x, \nu)\{\lambda \Re(x)+\mathfrak{Q}(x)\}, \\
\mathfrak{z}(0, \nu) & =\mathfrak{b}(\nu) \mathfrak{B}^{(0)}(\lambda, \nu), \\
\mathfrak{z}(1, \nu) & =-\mathfrak{b}(\nu) \mathfrak{B}^{(1)}(\lambda, \nu),
\end{aligned}
$$

may be looked upon as constituting a differential system for a pair of vectors $\mathfrak{b}(\nu)$, and $\mathfrak{z}(x, \nu)$, of the form

$$
\begin{aligned}
\mathfrak{b}(\nu) & \equiv\left(b_{1}(\nu), b_{2}(\nu)\right), \\
z(x, \nu) & \equiv\left(z_{1}(x, \nu), z_{2}(x, \nu)\right) .
\end{aligned}
$$

With $\lambda$ and $\nu$ at any specific values, this system will be characterized as the adjoint of the respective differential system (5.2) $\left({ }^{9}\right)$. As in the case of this latter, $\lambda$ and $\nu$ are to be considered as complex scalar parameters. The vector $\mathfrak{b}(\nu)$ will be referred to as the parametric vector, and of a pair of vectors (19.2) which together satisfy the equations of the system, the vector $z(x, \nu)$ will be called the solution $\left({ }^{10}\right)$.

Let $\mathfrak{V}(x, \lambda)$, as heretofore, be any nonsingular solution of the matrix equation (4.1). The general solution of the differential equation of the system (19.1) is then given by the formula

$$
z(x, \nu) \equiv \mathfrak{l}(\nu) \mathfrak{Y}^{-1}(x, \lambda),
$$

in which $\mathfrak{t}(\nu)$ is an arbitrary vector independent of $x$. Upon substituting this form into the boundary relations of the system, the vector $\mathfrak{l}(\nu)$ is found to be subject to the evaluations

$$
\begin{aligned}
& \mathfrak{f}(\nu)=\mathfrak{b}(\nu) \mathfrak{B}^{(0)}(\lambda, \nu) \mathfrak{V}(0, \lambda), \\
& \mathfrak{f}(\nu)=-\mathfrak{b}(\nu) \mathfrak{B}^{(1)}(\lambda, \nu) \mathfrak{Y}(1, \lambda) .
\end{aligned}
$$

(9) The comparative structure of the systems is somewhat better shown if the equations (5.2) are written in the form $\mathfrak{y}^{1}(x, \nu)=\{\lambda \Re(x)+\mathfrak{Q}(x)\} \mathfrak{y}(x, \nu), \mathfrak{B}^{0)}(\lambda, \nu) \mathfrak{y}(0, \nu)=\mathfrak{a}(\nu)$, $\mathfrak{B}^{\mathfrak{1})}(\lambda, \nu) \mathfrak{b}(1, \nu)=-\mathfrak{a}(\nu)$.

(10) This formulation of the adjoint differential system differs in some relatively minor details from that given by the author in the paper: The boundary problem of an ordinary linear differential system in the complex domain, Trans. Amer. Math. Soc. vol. 46 (1939) p. 165. It is obtainable therefrom, however, by setting $m=2, \eta_{0}=\eta_{1}=0, \eta_{2}=1$, and $z^{(2)}(x) \equiv-z^{(1)}(x)$ $z(x, \nu)$. 
The consistency of these evaluations, as may be seen by the elimination of $\mathfrak{f}(\nu)$, is contingent upon fulfillment of the condition

$$
\mathfrak{b}(\nu) \mathfrak{D}(\lambda, \nu)=\mathfrak{o},
$$

in which $\mathfrak{D}(\lambda, \nu)$ is precisely the matrix given by the formula (5.6). A solution of the system may, therefore, exist only in association with a parametric vector which satisfies the condition (19.5). Conversely, it is seen at once, every parametric vector which does satisfy this condition has a solution associated with it through the relations (19.4) and (19.3).

The choice $\mathfrak{b}(\nu)=\mathfrak{D}$ obviously satisfies the equation (19.5). It is, however, uniquely associated with the solution $z(x, \nu) \equiv \mathbf{0}$. This solution, which is thus always available, may properly be regarded as trivial, and to bar it from the further considerations the specification.

$$
\mathfrak{b}(\nu) \neq \mathfrak{o},
$$

will be imposed. Under this condition the possibility of fulfilling the relation (19.5), and hence the existence of a solution $z(x, \nu)$, is contingent upon the values of $\lambda$ and $\nu$, and the differential system (19.1) may accordingly be regarded as defining a family of boundary problems, precisely as such a family is defined by the system (5.2). The two families (19.1) and (5.2) will be defined to be adjoint.

Under the restriction (19.6), the equation (19.5) is solvable if and only if $\lambda$ is a value for which the matrix $\mathfrak{D}(\lambda, \nu)$ is singular. Such values of $\lambda$ are accordingly to be designated as characteristic values of the boundary problem (19.1). Since, as roots of the equation (5.7), they have already been identified as characteristic values of the boundary problem (5.2), it must be concluded that adjoint boundary problems have the same characteristic values. That every such value is of the same index, namely admits the same number of linearly independent solutions, for each of the two boundary problems follows also. For the numbers of linearly independent vectors $\mathfrak{c}^{(r)}(\nu)$ and $\mathfrak{b}^{(r)}(\nu)$ which satisfy the respective equations

$$
\begin{aligned}
\mathfrak{D}\left(\lambda_{r}, \nu\right) \mathfrak{c}^{(r)}(\nu) & =\mathfrak{o}, \\
\mathfrak{b}^{(r)} \nu \mathfrak{D}\left(\lambda_{r}, \nu\right) & =\mathfrak{D},
\end{aligned}
$$

at the characteristic value $\lambda_{r}$, are, of course, either both one or both two, according as the rank of the matrix $\mathfrak{D}\left(\lambda_{r}, \nu\right)$ is one or zero. If this rank is zero, it is clear that $\lambda_{r}$ must be of multiplicity at least two as a zero of the determinant $D(\lambda, \nu)$. The multiplicity of a characteristic value is, therefore, never exceeded by its index.

The solutions which are associated with any vectors $\mathfrak{c}^{(r)}(\nu)$ and $\mathfrak{b}^{(r)}(\nu)$ fulfilling the relations (19.7), are given, respectively, by the formula

$$
\mathfrak{y}^{(r)}(x, \nu) \equiv \mathfrak{Y}\left(x, \lambda_{r}\right) \mathfrak{c}^{(r)}(\nu),
$$


and by either one of the equivalent formulas

$$
\begin{aligned}
& \mathfrak{z}^{(r)}(x, \nu) \equiv \mathfrak{b}^{(r)}(\nu) \mathfrak{B}^{(0)}\left(\lambda_{r}, \nu\right) \mathfrak{W}\left(0, \lambda_{r}\right) \mathfrak{Y}^{-1}\left(x, \lambda_{r}\right), \\
& \mathfrak{z}^{(r)}(x, \nu) \equiv-\mathfrak{b}^{(r)}(\nu) \mathfrak{B}^{(1)}\left(\lambda_{r}, \nu\right) \mathfrak{W}\left(1, \lambda_{r}\right) \mathfrak{Y}^{-1}\left(x, \lambda_{r}\right) .
\end{aligned}
$$

For the purposes of subsequent deductions certain pairings of these solutions are advantageous, and are definable whenever the characteristic value concerned is of an index equal to its multiplicity.

Let $d_{i j}$ designate the elements of the matrix $\mathfrak{D}$, so that

$$
\mathfrak{D}(\lambda, \nu) \equiv\left(d_{i j}(\lambda, \nu)\right) \text {. }
$$

If $\lambda_{r}$ is a characteristic value of the index and multiplicity one, the relations

$$
d_{k l}\left(\lambda_{r}, \nu\right) \neq 0, \quad D_{\lambda}\left(\lambda_{r}, \nu\right) \neq 0,
$$

maintain if the subscripts $(k, l)$ are suitably chosen. The relations (19.7) are then in particular fulfilled by the vectors

$$
\begin{aligned}
& \mathfrak{c}^{(r)}(\nu)=\frac{1}{d_{k l}\left(\lambda_{r}, \nu\right)}\left(\begin{array}{c}
d_{k 2}\left(\lambda_{r}, \nu\right) \\
-d_{k 1}\left(\lambda_{r}, \nu\right)
\end{array}\right), \\
& \mathfrak{b}^{(r)}(\nu)=\frac{1}{D_{\lambda}\left(\lambda_{r}, \nu\right)}\left(-d_{2 l}\left(\lambda_{r}, \nu\right), d_{1 l}\left(\lambda_{r}, \nu\right)\right),
\end{aligned}
$$

and neither of these is the zero vector. With the evaluations (19.11), the solutions (19.8), (19.9) will be said to be a normal pair. If $\lambda_{\mathrm{s}}$ is a characteristic value of index and multiplicity two, it may conventionally be regarded as the pair of coincident values $\lambda_{\varepsilon}$ and $\lambda_{s+1}$. Since in this case the value

$$
\lim _{\lambda \rightarrow \lambda_{s}} \frac{D(\lambda, \nu)}{\left(\lambda-\lambda_{s}\right)^{2}}
$$

is not zero, whereas it is the determinant of the matrix

$$
\mathfrak{D}_{\lambda}\left(\lambda_{s}, \nu\right) \equiv\left(\lim _{\lambda \rightarrow \lambda_{s}} \frac{d_{i},(\lambda, \nu)}{\lambda-\lambda_{s}}\right),
$$

this matrix is nonsingular. It may then be verified that the determinations

$$
\begin{aligned}
\mathfrak{c}^{(s)}(\nu)=\left\{\mathfrak{I}_{\lambda}\left(\lambda_{s}, \nu\right)\right\}^{-1}\left(\begin{array}{l}
1 \\
0
\end{array}\right), & \mathfrak{b}^{(s)}(\nu)=(-1,0), \\
\mathfrak{c}^{(s+1)}(\nu)=\left\{\mathfrak{D}_{\lambda}\left(\lambda_{s+1}, \nu\right)\right\}^{-1}\left(\begin{array}{l}
0 \\
1
\end{array}\right), & \mathfrak{b}^{(s+1)}(\nu)=(0,-1),
\end{aligned}
$$

fulfill the relations (19.7), respectively for $r=s$ and $r=s+1$. With each of them the respective solutions (19.8), (19.9) will also be said to be a normal pair. No normal pairing of solutions will be defined in the instance of characteristic values whose multiplicities and indices are not equal. 
20. The generalized relation of bi-orthogonality. Under the normalization of the given boundary problem in $\$ 3$, and the construction of its imbedding family in $\S 5$, each element $v_{i k}(\lambda, \nu)$ of the matrices $\mathfrak{B}^{(0)}(\lambda, \nu), \mathfrak{B}^{(1)}(\lambda, \nu)$ is a polynomial in $\lambda$ of maximum degree $\tau_{i}$, and $\tau_{1} \geqq \tau_{2}$. It is readily seen in virtue of this, that the equations

$$
\mathfrak{B}^{(h)}\left(\lambda^{\prime}, \nu\right)=\mathfrak{B}^{(h)}\left(\lambda^{\prime \prime}, \nu\right)-\left(\lambda^{\prime \prime}-\lambda^{\prime}\right) \sum_{l=0}^{r_{1}-1}\left(\lambda^{\prime \prime}\right) \mathfrak{B}^{(h, l)}\left(\lambda^{\prime}, \nu\right), \quad h=0,1,
$$

implicitly define the matrices which are therein designated by $\mathfrak{B}^{(n, l)}\left(\lambda^{\prime}, \nu\right)$, and that any element $v_{i j}^{(n, l)}\left(\lambda^{\prime}, \nu\right)$ of such a matrix is a polynomial in $\lambda^{\prime}$ of degree at most $\tau_{i}-l-1$ if $\tau_{i}-l-1 \geqq 0$, and vanishes identically if $\tau_{i}-l-1<0$.

The relation

$$
\int_{0}^{1}\left\{z(x) \mathfrak{Y}^{\prime}(x)+z^{\prime}(x) \mathfrak{Y}(x)\right\} d x=z(1) \mathfrak{Y}(1)-z^{(0) \mathfrak{Y}(0)}
$$

is an evident identity. If the vector $z$ and the matrix $\mathfrak{Y}$ involved in it are taken respectively to be any solution $z^{p}(x, \nu)$ and the matrix $\mathfrak{V}(x, \lambda)$ which appears in the formula (19.8), the equations (19.1) and (4.1) may be used to give the resulting equality the form

$$
\begin{aligned}
\left(\lambda-\lambda_{p}\right) \int_{0}^{1} z^{(p)}(x, \nu) \Re(x) \mathfrak{V}(x, \lambda) d x & \\
& +\mathfrak{b}^{(p)}(\nu)\left\{\mathfrak{B}^{(0)}\left(\lambda_{p}, \nu\right) \mathfrak{W}(0, \lambda)+\mathfrak{B}^{(1)}\left(\lambda_{p}, \nu\right) \mathfrak{V}(1, \lambda)\right\}=0 .
\end{aligned}
$$

In this expression the matrices $\mathfrak{B}^{(h)}\left(\lambda_{p}, \nu\right)$ may be replaced by their equivalents as given by the formulas (20.1) with $\lambda^{\prime}=\lambda_{p}$ and $\lambda^{\prime \prime}=\lambda$. The subsequent multiplication on the right by any one of the vectors $\left(\lambda-\lambda_{p}\right)^{-1} c^{(q)}(\nu)$, it being assumed that $\lambda \neq \lambda_{p}$, results then in giving the relation the form

$$
\begin{aligned}
\int_{0}^{1} z^{(p)}(x, \nu) \Re(x) \mathfrak{V}(x, \lambda) \mathfrak{c}^{(q)}(\nu) d x & \\
-\mathfrak{b}^{(p)}(\nu) \sum_{l=0}^{\tau_{1}-1} \lambda^{l}\left\{\mathfrak{B}^{(0, l)}\left(\lambda_{p}, \nu\right) \mathfrak{V}(0, \lambda) \mathfrak{c}^{(q)}(\nu)\right. & \left.+\mathfrak{B}^{(1, l)}\left(\lambda_{p}, \nu\right) \mathfrak{W}(1, \lambda) \mathfrak{c}^{(q)}(\nu)\right\} \\
& =\frac{-1}{\lambda-\lambda_{p}} \mathfrak{b}^{(p)}(\nu) \mathfrak{D}(\lambda, \nu) \mathfrak{c}^{(q)}(\nu) .
\end{aligned}
$$

As $\lambda \rightarrow \lambda_{q}$ it follows from the formula (19.8) and the analyticity of the matrix $\mathfrak{V}(x, \lambda)$ as to $\lambda$, that

$$
\lim _{\lambda \rightarrow \lambda_{q}} \mathfrak{Y}(x, \lambda) \mathfrak{c}^{(q)}(\nu)=\mathfrak{y}^{(q)}(x, \nu) .
$$

It follows similarly from the first of the relations (19.7) that 


$$
\lim _{\lambda \rightarrow \lambda_{q}} \mathfrak{D}(\lambda, \nu) \mathfrak{c}^{(q)}(\nu)=\mathfrak{D} .
$$

If $\lambda_{p} \neq \lambda_{q}$, therefore, each member of the relation (20.3) approaches a limit as $\lambda \rightarrow \lambda_{q}$, and the limiting form of the relation as it is given below under (20.5)(11) may be regarded as established whenever the solutions $z^{(p)}(x, \nu)$ and $\mathfrak{y}^{(q)}(x, \nu)$ are associated with distinct characteristic values. It is to be shown that the relation (20.5) is valid also when $\lambda_{p}=\lambda_{q}$, provided the solutions involved are each a member of a normal pair.

When $\lambda_{p}=\lambda_{q}$, the limit of the right-hand member of the relation (20.3) is

$$
-\mathfrak{b}^{(p)}(\nu) \mathfrak{D}_{\lambda}\left(\lambda_{q}, \nu\right) \mathfrak{c}^{(q)}(\nu) .
$$

If $z^{(p)}(x, \nu)$ and $y^{(q)}(x, \nu)$ are not members of the same normal pair, the characteristic value in question is of the index two, and by the convention adopted in $\$ 19, p \neq q$. The vectors $\mathfrak{b}^{(p)}(\nu)$ and $\mathfrak{c}^{(q)}(\nu)$ are in this case evaluated by the formulas (19.13), with $(p, q)$ identified either with $(s, s+1)$ or with $(s+1, s)$. Under either alternative it is found directly that the limit (20.4) is 0 , and hence that the relation (20.5) below again maintains.

When $p=q$ the limit (20.4) is easily found to be 1 if the characteristic value is of the index two. The vectors $\mathfrak{b}^{(p)}(\nu)$ and $\mathfrak{c}^{(q)}(\nu)$ are then as given by the formulas (19.13) either with $p=q=s$, or with $p=q=s+1$, and the result is immediate. If the characteristic value is of the index one, the expression (20.4) for the limit is conveniently replaced by

$$
-\mathfrak{b}^{(p)}(\nu) \mathfrak{D}_{\lambda}\left(\lambda_{p}, \nu\right) \mathfrak{c}^{(p)}(\nu)-\frac{d_{k l}^{\prime}\left(\lambda_{p}, \nu\right) D\left(\lambda_{p}, \nu\right)}{d_{k l}\left(\lambda_{p}, \nu\right) D_{\lambda}\left(\lambda_{p}, \nu\right)},
$$

which is its equivalent, since $D\left(\lambda_{p}, \nu\right)=0$. The vectors $\mathfrak{b}^{(p)}(\nu)$ and $\mathfrak{c}^{(p)}(\nu)$ are in this instance evaluated by the formulas (19.11), and with these values the limit in question is found, as has been stated, to be 1 .

The solutions of adjoint boundary problems which are members of normal pairs thus fulfill the relations

$$
\begin{aligned}
& \int_{0}^{1} z^{(p)}(x, \nu) \Re(x) \mathfrak{y}^{(q)}(x, \nu) d x \\
& \quad-\mathfrak{b}^{(p)}(\nu) \sum_{l=0}^{\gamma_{1}-1} \lambda_{p}^{l}\left\{\mathfrak{B}^{(0, l)}\left(\lambda_{p}, \nu\right) \mathfrak{y}^{(q)}(0, \nu)+\mathfrak{B}^{(1, l)}\left(\lambda_{p}, \nu\right) \mathfrak{y}^{(q)}(1, \nu)\right\}=\delta_{p q} .
\end{aligned}
$$

It will be observed that in the absence of the indicated sums in their left-hand members, these relations reduce to the expression of a familiar property of weighted bi-orthogonality of the solutions involved. This reduction evidently maintains whenever $\tau_{1}=0$, namely whenever the boundary problem given is

(11) In which $\delta_{p q}=0$ if $p \neq \neq q, \delta_{p p}=1$. 
one in which the boundary relations are independent of the parameter $\lambda$. The relations (20.5) may, therefore, be looked upon as generally expressive of a property of the solutions of which bi-orthogonality is a specialization.

\section{Chapter 7}

\section{EXPANSIONS IN SERIES OF CHARACTERISTIC SOLUTIONS}

21. The formal expansions of arbitrary vectors. When the parameter $\nu$ is on a regular path, and $\nu \neq 0$, all characteristic values, as has been seen, satisfy the relation (15.6), and are therefore simple roots of the characteristic equation. Every such value is, therefore, of the same index as multiplicity, and the characteristic solutions of the adjoint boundary problems accordingly have the property that they may be adjusted to appear without exception as members of normal pairs. It is essential for the continuing discussion that this property be invariably present, namely also at $\nu=0$. Since the boundary problem is then as originally given, the inherence of the property in it must be a matter of assumption, and this it will be made by the following:

HyPOTHESIS 4. The given boundary problem is one for which every characteristic value is of an index equal to its multiplicity.

On the basis of this hypothesis it may, and will be, understood in the following, that the designations $z^{(p)}(x, \nu), y^{(p)}(x, \nu)$, are reserved to solutions of normal pairs.

If with any sequence of scalar coefficients $\alpha_{p}(\nu), p=1,2,3, \cdots$, the series of characteristic solutions in the equation

$$
\sum_{p=1}^{\infty} \alpha_{p}(\nu) \mathfrak{y}^{(p)}(x, \nu)=\mathfrak{f}(x, \nu),
$$

is convergent uniformly on the interval $0 \leqq x \leqq 1$, and defines there the vector $f(x, \nu)$ as shown, and if over and above that the related series in the equations

$$
\begin{aligned}
& \sum_{p=1}^{\infty} \alpha_{p}(\nu) \lambda_{p}^{l} \eta^{(p)}(0, \nu)=f^{(0, l)}(\nu), \\
& \sum_{p=1}^{\infty} \alpha_{p}(\nu) \lambda_{p}^{l} \eta^{(p)}(1, \nu)=f^{(1, l)}(\nu), \quad l=1,2,3, \cdots,\left(\tau_{1}-1\right),
\end{aligned}
$$

also converge and define the indicated vectors $f^{(i, l)}(\nu)$, the coefficients in question necessarily fulfill in turn the relations

$$
\begin{aligned}
& \alpha_{p}(\nu)= \int_{0}^{1} \mathfrak{z}^{(p)}(\xi, \nu) \Re(\xi) \mathfrak{f}(\xi, \nu) d \xi \\
&-\mathfrak{b}^{(p)}(\nu) \sum_{l=0}^{\tau_{1}-1}\left\{\mathfrak{B}^{(0, l)}\left(\lambda_{p}, \nu\right) \mathfrak{f}^{(0, l)}(\nu)+\mathfrak{B}^{(1, l)}\left(\lambda_{p}, \nu\right) \mathfrak{f}^{(1, l)}(\nu)\right\} \\
& p=1,2,3, \cdots,
\end{aligned}
$$


in which the abbreviations

$$
\mathfrak{f}^{(0,0)}(\nu)=\mathfrak{f}(0, \nu), \quad \mathfrak{f}^{(1,0)}(\nu)=\mathfrak{f}(1, \nu),
$$

have been used. This may be established, simply by substituting for the respective vectors $f(x, \nu)$ and $f^{(i, l)}(\nu)$ in the formulas (21.3), their equivalent series (21.1), (21.2), interchanging the orders of integration and summation, and applying then the relations (20.5).

With the formulas (21.3) thus at hand, the stated conditional basis, upon which their relation with the equations (21.1) and (21.2) has been made evident, may be abandoned. If with an arbitrary vector $f(x, \nu)$, whose components are integrable as to $x$, an arbitrary auxiliary set of vectors $f^{(j, l)}(\nu)$ is taken to be associated, the formulas (21.3) relate to these vectors a sequence of scalars $\alpha_{p}(\nu)$ as indicated. With these scalars as coefficients, the series of characteristic solutions

$$
\sum_{p=1}^{\infty} \alpha_{p}(\nu) \mathfrak{y}^{(p)}(x, \nu)
$$

is formally determined, and will be referred to in short as an expansion of the vector $f(x, \nu)$. Inasmuch as this definition of an expansion is wholly formal, the question of the convergence of such an expansion must manifestly be regarded as an open one. More generally, the amenability of any given expansion to evaluation by "means of summability" of any specific type would be a matter calling for investigation, as would also all questions hinging upon the relation which any value thus conventionally assigned to an expansion may bear to the original generating vector $f(x, \nu)$.

22. The expansions as series of residues. In terms of any analytic nonsingular solution of the matrix equation (4.1), and the corresponding matrix $\mathfrak{D}(\lambda, \nu)$ given by the relation (5.6), let $\mathfrak{S}(x, \xi, \lambda, \nu)$, which is to be known as the "Green's" matrix, be defined by the formulas

$$
\begin{array}{r}
\mathfrak{S}(x, \xi, \lambda, \nu) \equiv \mathfrak{V}(x, \lambda) \mathfrak{D}^{-1}(\lambda, \nu) \mathfrak{B}^{(0)}(\lambda, \nu) \mathfrak{V}(0, \lambda) \mathfrak{Y}^{-1}(\xi, \lambda), \\
\text { for } 0 \leqq \xi \leqq x, \\
\mathfrak{S}(x, \xi, \lambda, \nu) \equiv-\mathfrak{V}(x, \lambda) \mathfrak{D}^{-1}(\lambda, \nu) \mathfrak{B}^{(1)}(\lambda, \nu) \mathfrak{V}(1, \lambda) \mathfrak{Y}^{-1}(\xi, \lambda), \\
\text { for } x<\xi \leqq 1 .
\end{array}
$$

At any set of arguments $(x, \xi)$ this matrix, as a function of $\lambda$, is analytic except at the characteristic values, where singularities are introduced through at least some of the elements of the matrix $\mathfrak{D}^{-1}(\lambda, \nu)$. These singularities are poles, as may be seen from the formula

$$
\mathfrak{D}^{-1}(\lambda, \nu) \equiv\left(\begin{array}{rr}
d_{22}(\lambda, \nu) / D(\lambda, \nu) & -d_{12}(\lambda, \nu) / D(\lambda, \nu) \\
-d_{21}(\lambda, \nu) / D(\lambda, \nu) & d_{11}(\lambda, \nu) / D(\lambda, \nu)
\end{array}\right)
$$


and moreover poles of the first order whenever, as is here the case, each characteristic value is of a multiplicity equal to its index. The residues are therefore non-vanishing, and may, in the case of the Green's matrix, be computed from either one of the formulas (22.1), since the difference of the right-hand members of these formulas is the matrix $\mathfrak{Y}(x, \lambda) \mathfrak{Y}^{-1}(\xi, \lambda)$, and is thus analytic. With the notational choice of the prefix "res ${ }_{p}$ " to indicate, for any matrix to which it is applied, the residue at the characteristic value $\lambda_{p}$, it follows, therefore, from the formulas (22.1) that

$$
\operatorname{res}_{p} \mathfrak{S}(x, \xi, \lambda, \nu)=\mathfrak{Z}\left(x, \lambda_{p}\right)\left\{\operatorname{res}_{p} \mathfrak{D}^{-1}(\lambda, \nu)\right\} \mathfrak{B}^{(0)}\left(\lambda_{p}, \nu\right) \mathfrak{V}\left(0, \lambda_{p}\right) \mathfrak{Y}^{-1}\left(\xi, \lambda_{p}\right) .
$$

With any choice of the characteristic value $\lambda_{p}$, and with $\lambda$ distinct from it but in a suitably small neighborhood of it, the identity

$$
\left(\lambda-\lambda_{p}\right) \mathfrak{D}^{-1}(\lambda, \nu) \equiv\left\{\frac{1}{\lambda-\lambda_{p}} \mathfrak{D}(\lambda, \nu)\right\}^{-1},
$$

is an obvious one. Its limiting form as $\lambda \rightarrow \lambda_{p}$ is contingent upon the multiplicity of this characteristic value. If $\lambda_{p}$ is simple, the formula (22.2) shows the limit to be

$$
\operatorname{res}_{p} \mathfrak{D}^{-1}(\lambda, \nu)=\frac{1}{D_{\lambda}\left(\lambda_{p}, \nu\right)}\left(\begin{array}{rr}
d_{22}\left(\lambda_{p}, \nu\right) & -d_{12}\left(\lambda_{p}, \nu\right) \\
-d_{21}\left(\lambda_{p}, \nu\right) & d_{11}\left(\lambda_{p}, \nu\right)
\end{array}\right)
$$

whereas it may be seen directly when the characteristic value is multiple, say when $\lambda_{p}=\lambda_{p+1}$, that the limit is

$$
\operatorname{res}_{p} \mathfrak{D}^{-1}(\lambda, \nu)=\left\{\mathfrak{D}_{\lambda}\left(\lambda_{p}, \nu\right)\right\}^{-1} .
$$

With these two alternatives there are associated respectively the formulas (19.11) and (19.13), and from them it may be verified that

$$
\operatorname{res}_{p} \mathfrak{D}^{-1}(\lambda, \nu)=\sum_{\lambda_{h}=\lambda_{p}}\left\{-\mathfrak{c}^{(h)}(\nu) \mathfrak{b}^{(h)}(\nu)\right\}\left({ }^{12}\right),
$$

in which the sum indicated upon the right consists of the single term for which $h=p$, or of the pair of terms for which $h=p, p+1$, according as $\lambda_{p}$ is simple, or $\lambda_{p}=\lambda_{p+1}$. The substitution of the result (22.4) into the formula (22.3) leads, in virtue of the relations (19.8) and (19.9), to the conclusion that

$$
\operatorname{res}_{p} B(x, \xi, \lambda, \nu)=\sum_{\lambda_{h}=\lambda_{p}}\left\{-\mathfrak{y}^{(h)}(x, \nu) \mathfrak{z}^{(h)}(\xi, \nu)\right\} .
$$

Consider now, in the case of any expansion (21.5), the term, or pair of terms, associated with any characteristic value. Since $\alpha_{p}(\nu)$ is a scalar, and

(12) The vectors are to be regarded as matrices for the purposes of the multiplications indicated. Thus $\left\{\mathfrak{c}^{(h)}(\nu) \mathfrak{b}^{(h)}(\nu)\right\}$ is a square matrix. 
because of the evaluation (19.8), the formula (21.3) leads directly to the equation

$$
\begin{aligned}
\alpha_{p}(\nu) \mathfrak{y}^{(p)}(x, \nu) & =\int_{0}^{1} \mathfrak{y}^{(p)}(x, \nu) \mathfrak{z}^{(p)}(\xi, \nu) \Re(\xi) \mathfrak{f}(\xi, \nu) d \xi \\
& -\mathfrak{V}\left(x, \lambda_{p}\right) \mathfrak{c}^{(p)}(\nu) \mathfrak{b}^{(p)}(\nu) \sum_{l=0}^{\gamma_{1}-1}\left\{\mathfrak{B}^{(0, l)}\left(\lambda_{p}, \nu\right) \mathfrak{f}^{(0, l)}(\nu)+\mathfrak{B}^{(1, l)}\left(\lambda_{p}, \nu\right) \mathfrak{f}^{(1, l)}(\nu)\right\},
\end{aligned}
$$

and this, together with the results (22.4) and (22.5), yields the relation

$$
\begin{aligned}
& \sum_{\lambda_{h}=\lambda_{p}} \alpha_{h}(\nu) \mathfrak{y}^{(h)}(x, \nu)=-\int_{0}^{1} \operatorname{res}_{p} \circlearrowleft(x, \xi, \lambda, \nu) \Re(\xi) \mathfrak{f}(\xi, \nu) d \xi \\
& +\operatorname{res}_{p}\left\{\mathfrak{V}(x, \lambda) \mathfrak{D}^{-1}(\lambda, \nu) \sum_{l=0}^{\tau_{1}-1}\left[\mathfrak{B}^{(0, l)}(\lambda, \nu) \mathfrak{f}^{(0, l)}(\nu)+\mathfrak{B}^{(1, l)}(\lambda, \nu) \mathfrak{f}^{(1, l)}(\nu)\right]\right\} .
\end{aligned}
$$

The terms of any expansion (21.5) are thus expressible as residues in the complex plane. It follows from this, of course, that any finite set of such terms may be summed by a suitably designed contour integral as to $\lambda$, the contour of integration being chosen to avoid the characteristic values, and to enclose precisely those which are associated with the terms of the set in question. In $\$ 17$ a certain infinite sequence of contours $C_{n}$ was defined, any one of the sequence, $C_{n}$, enclosing precisely those characteristic values $\lambda_{p}$ for which $\left|\lambda_{p}\right| \leqq\left|\lambda_{n}\right|$. If for the values of $n$ there concerned, the initial partial sums of the expansion (21.5) are denoted by $8(x, \nu, n)$, in the manner

$$
\mathfrak{B}(x, \nu, n) \equiv \sum_{p=1}^{n} \alpha_{p}(\nu) \mathfrak{y}^{(p)}(x, \nu)
$$

it follows that these sums are evaluated respectively by the formulas

$$
\begin{aligned}
& \mathscr{B}(x, \nu, n)=\frac{-1}{2 \pi i} \int_{0}^{1} \int_{C_{n}} \mathfrak{S}(x, \xi, \lambda, \nu) \Re(\xi) \mathfrak{f}(\xi, \nu) d \lambda d \xi \\
& +\sum_{l=0}^{\tau_{1}-1} \frac{1}{2 \pi i} \int_{C_{n}} \mathfrak{V}(x, \lambda) \mathfrak{D}^{-1}(\lambda, \nu)\left[\mathfrak{B}^{(0, l)}(\lambda, \nu) \mathfrak{f}^{(0, l)}(\nu)+\mathfrak{B}^{(1, l)}(\lambda, \nu) \mathfrak{f}^{(1, l)}(\nu)\right] d \lambda .
\end{aligned}
$$

23. On matters of convergence, divergence, and summability. When the parameter $\nu$ is on a regular path, and $\nu \neq 0$, the formulas (21.3) associate with any suitable vector $f(x, \nu)$ an expansion (21.5) in solutions of a boundary problem of the regular type. Such expansions, both in the vector form here in question, and in the alternative scalar form $\left({ }^{13}\right)$, are familiar, and it is known

(13) For a discussion of the relations between the scalar and vector formulations, cf. the author's paper, The expansion problem in the theory of ordinary linear differential systems of the second order, Trans. Amer. Math. Soc. vol. 31 (1929) p. 887. 
that their properties are broadly exemplified by those of the classical Fourier's series. In particular, if $x$ is an interior point of the basic interval, and if in some neighborhood of it the components of the generating vector $f(x, \nu)$ are of bounded variation, the expansion converges at this point to the average value, namely

$$
\lim _{n \rightarrow \infty} 8(x, \nu, n)=(1 / 2)\{\mathfrak{f}(x+, \nu)+\mathfrak{f}(x-, \nu)\}, \quad \nu \neq 0\left({ }^{14}\right) .
$$

These statements, on the other hand, do not ordinarily apply when $\nu=0$. The expansions are then relative to the given boundary problem, which is highly irregular, and little theory of such expansions is known. Indeed, as to boundary problems of the second order-the only ones here immediately pertinent-all highly irregular cases that have been analyzed at all are subsumable in the scalar form

$$
\begin{aligned}
y^{\prime \prime}(x)-(2 \lambda \cos p \pi / q) y^{\prime}(x)+\lambda^{2} y(x) & =0, \\
(1-a) y(0)+a y^{\prime}(0) & =0, \\
b_{1} y(0)+b_{2} y^{\prime}(0)+b_{3} y(1)+b_{4} y^{\prime}(1) & =0,
\end{aligned}
$$

with constant coefficients, and in particular with $a$ equal to either 0 or 1 , and with $p$ and $q$ relatively prime integers $\left({ }^{15}\right)$. Moreover, definitive results (uniform convergence), even for the expansions based upon these restricted systems, have been obtained only for highly specialized generating functions, specifically only when these functions are of the structure

$$
f(x) \equiv x^{1+a} \phi\left(x^{q}\right),
$$

with $\phi(z)$ some analytic function of the complex variable $z$ which is bounded in the circle $|z|<1\left({ }^{16}\right)$. The disparity between theorems such as these, and those which comprise the theory of expansions relative to regular boundary problems needs no emphasis.

That the expansions associated with highly irregular boundary problems are in general divergent, even when the generating functions are analytic, is observable from the simplest of explicit instances. Thus the expansion generated by the function $f(x) \equiv 1$ relative to the boundary problem (23.2) with $a=0, b_{4}=1, b_{j}=0, j=1,2,3$, is found to be

$$
\sum_{n=1}^{\infty}\left\{e^{x(n \pi-c) \cot c} \frac{\sin (n \pi-c) x}{n \pi-c}+e^{-x(n \pi+c) \cot c} \frac{\sin (n \pi+c) x}{n \pi+c}\right\}
$$

(14) A proof of this is also implicit in the deductions of Chapter 8 below.

(15) J. I. Vass, loc. cit.

(16) For highly irregular boundary problems of order higher than the second, the known expansion theorems are similar and of comparable generality. They refer exclusively to boundary problems in which the differential equation is of the form $y^{(n)}(x)+\left\{\lambda^{n}+\sigma(x)\right\} y(x)=0, n \geqq 3$, or some specialization of this form. Cf. J. W. Hopkins and L. E. Ward, loc. cit. 
with $c=p \pi / q$. On the interval $0<x<1$ the terms of this series fail to approach zero as $n \rightarrow \infty$. The generating function $f(x) \equiv x^{k+1}$ leads to a similar result whenever $k$ is not an integral multiple of $q$.

It will be clear, even from these fragmentary citations, that potentialities of fruitfulness in application to expansions of the type (21.5), with generating vectors that are in any broad sense arbitrary, are to be sought only in schemes of evaluation which refer to, and are based upon, some notion more general than that of ordinary convergence. In the following, two such modes of summation, each one of a familiar pattern, are to be defined.

To begin with, since the boundary problem originally given is identified with the parameter value $\nu=0$, the expansion generated relative to it by a vector $f(x)$, is obtainable from the formula (21.5) by the identification of $f(x)$ with $f(x, 0)$, and is, thus

$$
\sum_{p=1}^{\infty} \alpha_{p}(0) \mathfrak{y}^{(p)}(x, 0)
$$

Now under the hypotheses to which the boundary problems have already been subjected, the characteristic values, and hence also the characteristic solutions, are continuous as functions of $\nu$ along any regular path, inclusive of the terminal point $\nu=0$. If the vectors $f(x, \nu)$ and $f^{(i, l)}(\nu)$ are, therefore, likewise taken to be continuous in $\nu$, and such that

$$
\lim _{\sim \rightarrow 0} \mathfrak{f}(x, \nu)=\mathfrak{f}(x),
$$

it is evident from the formulas (21.3) that each individual term of the expansion (21.5) is continuous and approaches the respective term of the expansion (23.3) as a limit when $\nu \rightarrow 0$. This latter series may, therefore, be regarded as formally given by the expression

$$
\lim _{n \rightarrow \infty} \lim _{n \rightarrow 0} 8(x, \nu, n) \text {. }
$$

The expansion (23.3) is now to be defined as summable by the "means $A$ " to the value

$$
\lim _{\nu \rightarrow 0} \lim _{n \rightarrow \infty} z(x, \nu, n),
$$

if and when with some determination of $f(x, \nu)$ and $f^{(j, l)}(\nu)$ as vectors continuous in $\nu$ and fulfilling the relation (23.4), the limit (23.5) exists.

The notion of summation basically involved in these means, will be recognized as that which similarly underlies the classical means identified with the names of Abel and Borel. For these latter may be looked upon as evaluating a series

$$
\sum_{p=1}^{\infty} u_{p}(x)
$$


respectively, by the limits

and

$$
\lim _{\nu \rightarrow 0^{+}} \lim _{n \rightarrow \infty} \sum_{p=1}^{n}(1-\nu)^{p} u_{p}(x),
$$

$$
\lim _{\nu \rightarrow 0^{+}} \lim _{n \rightarrow \infty} \sum_{p=1}^{n}\left\{1-e^{1 / \nu} \sum_{j=1}^{p} \frac{\nu^{-j}}{j !}\right\} u_{p}(x) .
$$

By the use of the relations (23.1) and (23.4) in conjunction with the expression $(23,5)$, it will be evident that the deductions of the preceding sections have effectively established the following facts.

THEOREM. The expansion (23.3), generated by an integrable vector $f(x)$, is summable by the means $A$ to the value

$$
(1 / 2)\{\mathfrak{f}(x+)+\mathfrak{f}(x-)\} \text {, }
$$

at any point $x$ of the interval $0<x<1$, whenever $\mathrm{f}(x)$ is such as to admit at that point of representation in the manner (23.4), by a vector $f(x, \nu)$ which for every $\nu$ on a regular path other than $\nu=0$, fulfills the conditions

(i) that it is continuous in $\nu$;

(ii) that its expansions relative to regular boundary problems converge to the value

$$
(1 / 2)\{f(x+, \nu)+f(x-, \nu)\} .
$$

Since the role of $f(x, \nu)$ in this theorem may in particular be taken by the vector $f(x)$ itself, provided it fulfills the condition (ii), the following specialization of the theorem is evident.

COROLLARY. The expansion of an integrable vector $f(x)$ relative to the highly irregular boundary problem is summable by the means $A$ to the value (23.7), whenever $f(x)$ is such that its expansions relative to regular boundary problems converge to that value.

A second scheme of summation alternative to that described above may be defined in the following manner. value

The expansion (23.3) shall be said to be summable by the "means $B$ " to the

$$
\lim _{n \rightarrow \infty} 8\left(x, \nu_{n}^{\prime}, n\right)
$$

if

(i) with the role of $f(x, \nu)$ taken by the vector $f(x)$ itself, and the vectors $f^{(j, l)}$ independent of $\nu$;

(ii) with the points $\nu_{1}^{\prime}, \nu_{2}^{\prime}, \nu_{3}^{\prime}, \cdots$ on some regular path of $\nu$; and

(iii) with 


$$
\lim _{p \rightarrow \infty} \nu_{p}^{\prime}=0
$$

the limit (23.8) exists.

The means for summation of an expansion as thus defined may be seen without difficulty to bear in principle a resemblance to the classical means of Cesàro and Riesz. For these latter may be formulated respectively as assigning to a series (23.6) the evaluations

and

$$
\lim _{n \rightarrow \infty} \sum_{p=1}^{n}\left\{1-(p-1) \nu_{n}^{\prime}\right\} u_{p}(x), \text { with } \nu_{n}^{\prime}=1 / n,
$$

$$
\lim _{n \rightarrow \infty} \sum_{p=1}^{n}\left\{1-\omega(p) \nu_{n}^{\prime}\right\} u_{p}(x) \text {, with } \nu_{n}^{\prime}=1 / \omega(n),
$$

the function $\omega(n)$ being positive, increasing and unbounded.

Of the two schemes thus described, the means $B$ may be characterized as providing a subtler mode of summation than the means $A$, in much the same sense as the means of Cesàro may be regarded as less drastic than those of Borel. It is only consistent with this, that no inference of summability of a highly irregular expansion by the means $B$ is readable from the deductions already made. It is upon this point that the continuing discussion is focused.

\section{Chapter 8}

\section{The sUmmaBility of THE EXPansions By the MEANS $B$}

24. The formula for the partial sums. For any index $n$ which identifies a contour of the sequence $C_{n}$ defined in $\$ 17$, the terms of an expansion that correspond to the first $n$ characteristic values, are summed by the formula (22.8). The role of this formula in any analysis of the expansion is, of course, a central one; its convergence as $n \rightarrow \infty$, with either $\nu$ fixed or $\nu$ suitably dependent upon $n$, being tantamount respectively to the convergence or the summability of the expansion. The convergence of these sums, with appropriately disposed parameter values, must, therefore, in due course be considered. Preparatory to this, however, it is to be shown in this section that the formula (22.8) may be expressed in such a manner as to display, among other things, the fact that its elements are bounded as to $n$ when $\nu$ is bounded from zero. This is obscured in the formula as it stands, due to the fact that certain of its matrix factors have elements that are polynomials in $\lambda$, while others involve exponentials each one of which is clearly unbounded for some range of $\arg \lambda$ as $n \rightarrow \infty$.

The Green's matrix has been defined by the formula (22.1). For the purpose of giving alternative expressions for it, let the matrices $\Im_{r}$ be defined for all subscripts $r$ by the formulas 


$$
\begin{array}{lc}
\Im_{1}=\left(\begin{array}{ll}
0 & 0 \\
0 & 0
\end{array}\right), & \Im_{2}=\left(\begin{array}{ll}
1 & 0 \\
0 & 0
\end{array}\right), \\
\Im_{3}=\left(\begin{array}{ll}
1 & 0 \\
0 & 1
\end{array}\right), & \Im_{4}=\left(\begin{array}{ll}
0 & 0 \\
0 & 1
\end{array}\right), \\
\Im_{h}=\Im_{l}, \quad \text { if } & h \equiv l(\bmod 4),
\end{array}
$$

and let the relations

$$
\Im^{(r)} \equiv\left\{\begin{aligned}
\Im_{r+2}, & \text { when } 0 \leqq \xi \leqq x, \\
-\Im_{r}, & \text { when } \quad x<\xi \leqq 1,
\end{aligned}\right.
$$

specify the matrices $\Im^{(r)}(x, \xi)$. It is then a matter of simple verification that, irrespective of the choice of $r$, the formulas (22.1) are replaceable by the relation

$(24.3) \quad \mathbb{S}=\mathfrak{Y}(x) \mathfrak{\Im}^{(r)} \mathfrak{Y}^{-1}(\xi)+\mathfrak{V}(x) \mathfrak{D}^{-1}\left\{\mathfrak{B}^{(0)} \mathfrak{Y}(0) \Im_{r}-\mathfrak{B}^{(1)} \mathfrak{Y}(1) \Im_{r+2}\right\} \mathfrak{Y}^{-1}(\xi)\left({ }^{17}\right)$.

In virtue of the formula (5.6), the relation (24.3) is independent of the choice of $\mathfrak{Y}(x, \lambda)$ as a nonsingular solution of the equation (4.1). This solution may, therefore, be chosen at any value of $\lambda$ to be one to which the formulas (4.8), (4.9) apply. It will be supposed throughout that the solution $\mathfrak{V}(x, \lambda)$ is always so chosen. The elements $a_{i l}(\lambda, \nu)$, given by the relations (5.3) and (5.9), are then specific, and the matrix $\mathfrak{D}(\lambda, \nu)$ is subject to the formula (5.10).

Let the matrix $\mathfrak{D}_{0}(\lambda, \nu)$ be specified by the definition

$$
\mathfrak{D}_{0} \equiv\left(\begin{array}{rr}
a_{22}+a_{24} e^{\lambda \Gamma_{2}} & -a_{22}-a_{14} e^{\lambda \Gamma_{2}} \\
-a_{21}-a_{23} e^{\lambda \Gamma_{1}} & a_{11}+a_{13} e^{\lambda \Gamma_{1}}
\end{array}\right),
$$

and let $D_{0}$ designate its determinant. A comparison with the formula (5.10) yields then the evaluations

$$
\begin{aligned}
\mathfrak{D}^{-1} & =\frac{1}{D_{0}} \mathfrak{D}_{0} \cdot\left(\delta_{i j} \lambda^{-\tau_{i}}\right), \\
D & =\lambda^{\tau_{1}+\tau_{2}} D_{0} .
\end{aligned}
$$

Now it was observed in $\S 20$, that the elements of the matrices $\mathfrak{B}^{(h, l)}(\lambda, \nu)$, which occur in the formula (22.8), are polynomials in $\lambda$ of maximum degree $\tau_{i}-l-1$. In accordance with this, the matrices $\mathfrak{W}^{(h, l)}(\lambda, \nu)$, as defined by the relations

$$
\mathfrak{W}^{(h, l)} \equiv \lambda^{l+1}\left(\delta_{i j} \lambda^{-\tau_{i}}\right) \mathfrak{B}^{(h, l)}, \quad h=0,1 ; l=0,1,2, \cdots,\left(\tau_{1}-1\right),
$$

have elements that are polynomials in $1 / \lambda$, and it is clear that in terms of them

(17) Throughout the remaining discussion the explicit indications of functional arguments will be curtailed in the interest of simplicity in the formulas. Those variables that do not require current attention will therefore frequently be omitted. 


$$
\mathfrak{D}^{-1} \mathfrak{B}^{(h, l)}=\frac{1}{\lambda^{l+1} D_{0}} \mathfrak{D}_{0} \mathfrak{W}^{(h, l)} .
$$

Since by the relations (4.8), (5.3), (5.9), and (24.5) the evaluations

$$
\begin{aligned}
\mathfrak{D}^{-1} \mathfrak{B}(0) & \mathfrak{Y}(0)=\frac{1}{D_{0}} \mathfrak{D}_{0}\left(a_{i j}\right), \\
\mathfrak{D}^{-1} \mathfrak{B}^{(1)} \mathfrak{Y}(1) & =\frac{1}{D_{0}} \mathfrak{D}_{0}\left(a_{i, j+2}\right) \mathfrak{F}(1),
\end{aligned}
$$

also maintain, it will be recognized that the formula (24.3) is alternatively expressible in the form

$$
\mathfrak{S}=\mathfrak{Y}(x) \mathfrak{\Im}^{(r)} \mathfrak{Y}^{-1}(\xi)+\mathfrak{Y}(x) \frac{1}{D_{0}} \mathfrak{D}_{0}\left\{\left(a_{i j}\right) \Im_{r}-\left(a_{i, j+2}\right) \mathfrak{E}(1) \mathfrak{\Im}_{r+2}\right\} \mathfrak{Y}^{-1}(\xi) .
$$

The elements of the matrices $\mathfrak{B}(x, \lambda)$ and $\mathfrak{P}^{-1}(\xi, \lambda)$, which enter into this through the solution $\mathfrak{V}$ and its inverse, and hence also the elements of the matrices (5.9), are, as has been observed in $\$ 5$, asymptotically representable by formal power series in negative powers of $\lambda$. Through the relations (24.7) and (24.8), the expression of the integrands in the formula (22.8) without the utilization of any positive powers of $\lambda$ has thus been attained.

For the further analysis of these formulas, let the matrices $\mathfrak{V}_{r}(x, \lambda)$ be defined, for all indices $r$, by the formula

$$
\mathfrak{Y}_{r}(x, \lambda) \equiv \mathfrak{Y}(x, \lambda)\left\{\Im_{r} \mathfrak{F}^{-1}(1)+\Im_{r+2}\right\}
$$

The relations

$$
\begin{aligned}
\mathfrak{V} & =\mathfrak{V}_{r}\left\{\mathfrak{E}(1) \Im_{r}+\Im_{r+2}\right\}, \\
\mathfrak{V}^{-1} & =\left\{\Im_{r+2} \mathfrak{F}^{-1}(1)+\Im_{r}\right\} \mathfrak{Y}_{r+2}^{-1},
\end{aligned}
$$

follow at once, and as a consequence the formulas (24.8), (24.7) may be rewritten into the forms

$$
\begin{gathered}
\left(\mathfrak{S}=\mathfrak{Y}(x) \mathfrak{I}^{(r)} \mathfrak{Y}^{-1}(\xi)+\mathfrak{Y}_{r}(x) \mathfrak{U}_{r} \mathfrak{A}_{r} \mathfrak{Y}_{r+2}^{-1}(\xi),\right. \\
\mathfrak{Y}(x) \mathfrak{D} \mathfrak{B}^{(h, l)}=\frac{1}{\lambda^{l+1}} \mathfrak{Y}_{r}(x) \mathfrak{U}_{r} \mathfrak{W}^{(h, l)},
\end{gathered}
$$

in which

$$
\begin{aligned}
\mathfrak{A}_{r} & \equiv\left\{\left(a_{i j}\right) \Im_{r}-\left(a_{i, j+2}\right) \Im_{r+2}\right\}, \\
\mathfrak{U}_{r} & \equiv \frac{1}{D_{0}}\left\{\mathfrak{E}(1) \Im_{r}+\Im_{r+2}\right\} \mathfrak{D}_{0} .
\end{aligned}
$$


On the contours of the sequence $C_{n}$, let the arcs $C_{n r}$ be defined by the relations

$$
-\pi / 2-\arg \Gamma_{r-1} \leqq \arg \lambda \leqq-\pi / 2-\arg \Gamma_{r} .
$$

Any two contiguous arcs of this set comprise a semicircle, and those associated with any four successive values of $r$ constitute a complete contour. Upon associating with each arc $C_{n r}$ the respective evaluations (24.11), the formula (22.8) may now finally be expressed in the form

$$
\mathfrak{B}(x, \nu, n)=8_{0}(x, n)+\xi_{1}(x, \nu, n)+\xi_{2}(x, \nu, n),
$$

with

$$
\begin{aligned}
\mathfrak{g}_{0}(x, n) & \equiv \frac{-1}{2 \pi i} \sum_{r=1}^{4} \int_{0}^{1} \int_{C_{n r}}\left\{\mathfrak{V}(x) \mathfrak{Y}^{(r)} \mathfrak{Y}^{-1}(\xi)\right\} \Re(\xi) \mathfrak{f}(\xi) d \lambda d \xi \\
\mathfrak{B}_{1}(x, \nu, n) & \equiv \frac{-1}{2 \pi i} \sum_{r=1}^{4} \int_{0}^{1} \int_{C_{n r}} \mathfrak{V}_{r}(x) \mathfrak{U}_{r} \mathfrak{A} \mathfrak{Y}^{-1}(\xi) \Re(\xi) \mathfrak{f}(\xi) d \lambda d \xi \\
\mathbb{g}_{2}(x, \nu, n) & \equiv \frac{1}{2 \pi i} \sum_{r=1}^{4} \int_{C_{n r}} \mathfrak{V}_{r}(x) \mathfrak{U}_{r} \sum_{l=0}^{\tau_{1}-1} \lambda^{-l}\left\{\mathfrak{W}^{(0, l)} \mathfrak{F}^{(0, l)}+\mathfrak{W}^{(1, l)} \mathfrak{F}^{(1, l)}\right\} \frac{d \lambda}{\lambda}
\end{aligned}
$$

Of the matrices which enter into these formulas those designated by $\mathfrak{A}_{r}$ are shown by the relations (24.12) to involve no exponentials, and to be bounded for all large values of $|\lambda|$. The matrices $\mathfrak{u}_{r}$, on the other hand are less simply constructed. By the formula (18.1) and the second one of the relations (24.5), the equality

\begin{tabular}{|c|c|c|c|c|}
\hline$r$ & 1 & 2 & 3 & 4 \\
\hline & $\frac{B_{1}}{C_{1}(\nu)} \mathfrak{D}_{0}$ & 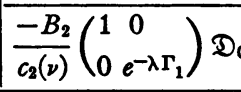 & $\frac{B_{3}}{c_{3}(\nu)}\left(\begin{array}{cc}e^{-\lambda \Gamma_{2}} & 0 \\
0 & e^{-\lambda \Gamma_{1}}\end{array}\right) D_{0}$ & $\frac{-B_{4}}{c_{4}(\nu)}\left(\begin{array}{cc}e^{-\lambda \Gamma_{2}} & 0 \\
0 & 1\end{array}\right) D_{0}$ \\
\hline
\end{tabular}

$$
\frac{1}{D_{0}}=\frac{(-1)^{r+1} B_{r}(\lambda, \nu)}{c_{r}(\nu)} e^{-\lambda\left(V_{r}-V_{1}\right)}
$$

is established, and in virtue of it the matrices in question are found to be explicitly as they are given by the table:

Consider the exponentials which occur in the elements of any one of the matrices

$$
\left\{\mathfrak{Y}(x) \Im^{(r)} \mathfrak{Y}^{-1}(\xi)\right\}, \quad \mathfrak{V}_{r}(x), \quad \mathfrak{V}_{r+2}^{-1}(\xi), \quad \mathfrak{u}_{r} .
$$

They are in every instance of a form which, in terms of the abbreviations

$$
\Gamma_{j}\left(x^{\prime}, x^{\prime \prime}\right) \equiv \Gamma_{j}\left(x^{\prime}\right)-\Gamma_{j}\left(x^{\prime \prime}\right),
$$

may be written as 


$$
e^{\lambda \Gamma_{\varepsilon}(\beta, \alpha)},
$$$$
s=r, r+1 \text {, }
$$

with the arguments $\alpha, \beta$ each taking some one of the values $0, \xi, x, 1\left({ }^{18}\right)$. Moreover, when they are so written, the subscripts $s$ being related to the index $r$ that identifies the arc of integration as is indicated in (24.19), then in every instance the relation $\beta \geqq \alpha$ is fulfilled. It will be shown that because of this every one of the exponentials in question is bounded over the range of integration for which it is involved.

From the formulas (2.5) and (2.7) it follows that when $\beta \geqq \alpha$, then

$$
\Gamma_{s}(\beta, \alpha)=\left|\Gamma_{s}(\beta, \alpha)\right| e^{\text {arg } \Gamma_{s} .}
$$

Under the substitutions given by the relations

$$
\lambda=\mp i \Lambda_{n} e^{\mp i \theta-1 \arg r_{s},}
$$

the upper or lower signs maintaining according as $s=r$ or $s=r+1$, the arc $C_{n r}$ corresponds to the range

$$
0 \leqq \theta \leqq \omega_{r-1},
$$

in which the angle $\omega_{r-1}$ is that given by the definition (11.8). The evaluations

$$
\left|e^{\lambda \Gamma_{s}(\beta, \alpha)}\right|=e^{-\Lambda_{n}\left|\Gamma_{s}(\beta, \alpha)\right| \sin \theta}, \quad \text { for } \beta \geqq \alpha,
$$

then show that on this range the exponentials concerned are bounded as to $n$. It follows that with the possible exception of $\mathfrak{U}_{r}$ the matrices (24.17) are all bounded on the respective arcs of integration involved in the formulas (24.15), and that unboundedness can inhere in the matrix $\mathfrak{u}_{r}$ only through the scalar factors indicated in the table (24.16).

By the relations (18.2) the scalar'functions $B_{r}(\lambda, \nu)$ are shown to be bounded as to $n$ uniformly as to $\nu$, provided the range of $\nu$ is restricted to the path segment $\left(\nu_{0}, \nu_{n}\right)$ when $\lambda$ is on the contour $C_{n}$. This condition is fulfilled in particular when $\nu$ is bounded from zero, and since in this case the boundedness of the coefficients $1 / c_{r}(\nu)$ is also assured, the uniform boundedness of the elements of the matrices $\mathfrak{U}_{r}(\lambda, \nu)$ follows. If $\nu$ is not bounded from zero, on the other hand, this conclusion may not be drawn, since it is the earmark of any highly irregular boundary problem that at least one coefficient $c_{r}(\nu)$ approaches zero with $\nu$. However, from the formulas (12.2) and (8.4) it is seen that at all events the functions $\nu / c_{r}(\nu)$ are bounded. It may accordingly be inferred that the elements of the matrices

$$
\left\{\nu \mathfrak{U}_{r}(\lambda, \nu)\right\},
$$

are uniformly bounded for $\nu$ on the path segment $\left(\nu_{0}, \nu_{n}\right)$ when $\lambda$ is on the $\operatorname{arc} C_{n r}$.

(18) It may be noted that $\Gamma_{j}\left(x^{\prime}\right)=\Gamma_{j}\left(x^{\prime}, 0\right)$, and that $\Gamma_{j}\left(x^{\prime}, x^{\prime \prime}\right)=\Gamma_{j+2}\left(x^{\prime \prime}, x^{\prime}\right)$, since $\Gamma_{i}\left(x^{\prime}\right)=-\Gamma_{j+2}\left(x^{\prime}\right)$. 
25. Lemmas. An analysis of the terms in the relation (24.14), as these are given by the respective formulas (24.15), may be based in large measure upon certain auxiliary deductions, of which some may be regarded as elementary, whereas others are appropriate adaptations of classical convergence theorems. The isolation and specific formulation of these deductions is a matter of evident convenience for their later applications. They will, therefore, be set forth in this section in the form of lemmas, in the interpretation of which it shall be understood that:

(i) Any interval designated by $(\alpha, \beta)$ is such that $0 \leqq \alpha<\beta \leqq 1$;

(ii) The symbol $\gamma_{n r}$ is a designation of the semicircle composed of the arcs $C_{n, r-1}$ and $C_{n r}$;

(iii) The range of the index $n$ is the sequence of integers for which the contours $C_{n}$ have been defined;

(iv) For any value of $n$ the range of the parameter $\nu$ is the segment $\left(\nu_{0}, \nu_{n}\right)$ of some chosen regular path;

(v) The range of the variable $\xi$ is in every instance an interval $\left(\xi_{1}, \xi_{2}\right)$ for which $0 \leqq \xi_{1}<\xi_{2} \leqq 1$;

(vi) Relative to any interval $\left(\xi_{1}, \xi_{2}\right)$ and any sequence of arcs $\gamma_{n r}$, the symbol $\mathfrak{M}(\xi, \lambda, \nu)$ is a generic designation for matrices whose elements $\mathfrak{m}_{i j}(\xi, \lambda, \nu)$ are uniformly bounded, namely which fulfill some set of relations

$$
\left|\mathfrak{m}_{i j}(\xi, \lambda, \nu)\right| \leqq \rho_{i j},
$$

in which the $\rho_{i j}$ are positive constants.

(vii) The symbol $\Im_{r}\left(\xi^{\prime}, \xi^{\prime \prime}, \xi, q\right)$ is defined by the formula

$$
\Im_{r}\left(\xi^{\prime}, \xi^{\prime \prime}, \xi, q\right)=\int_{\gamma_{n r}} e^{\lambda \Gamma_{r}\left(\xi^{\prime}, \xi^{\prime \prime}\right)} \mathfrak{M}(\xi, \lambda, \nu) d \lambda / \lambda^{q} .
$$

LEMMA 1. The elements of the matrix

$$
\int_{\gamma_{n r}} \mathfrak{M}(\xi, \lambda, \nu) \frac{d \lambda}{\lambda^{q+1}}
$$

are bounded uniformly as to $\xi, \nu$ and $n$ if $q \geqq 0$, and if $q>0$ they approach zero uniformly as to $\xi$ and $\nu$, as $n \rightarrow \infty$.

The asserted facts are obvious in virtue of the relations (25.1).

LEMMA 2. If $\beta>\alpha$, the elements of the matrix

$$
\Im_{r}(\beta, \alpha, \xi, q)
$$

are bounded uniformly as to $\xi, \nu$ and $n$ if $q \geqq 0$, and if $q>0$ they approach zero uniformly as to $\xi$ and $\nu$, as $n \rightarrow \infty$.

Since any arc $C_{n r}$ is identified with the respective range (24.20), the relations 


$$
\sin \theta \geqq \frac{\sin \omega_{r-1-j}}{\omega_{r-1-j}} \theta,
$$$$
j=0,1 \text {, }
$$

maintain on the respective portions $C_{n, r-j}$ of the arc of integration $\gamma_{n r}$. By virtue of the evaluations (24.21), it is therefore seen that the elements of the matrix (25.4) are dominated by those of the sum

$$
\sum_{j=0}^{1} \Lambda_{n}^{1-q} \int_{0}^{\omega_{r-1-i}} \exp \left\{-\Lambda_{n}\left|\Gamma_{r}(\beta, \alpha)\right| \frac{\sin \omega_{r-1-j}}{\omega_{r-1-j}} \theta\right\} d \theta\left(\rho_{i j}\right) .
$$

An explicit integration shows these dominant elements to be at all events bounded as to $n$, and to approach zero if $q$ is positive.

LEMMA 3. The elements of the matrices

$$
\Im_{r}(\beta, \xi, \xi, q), \quad \text { with } \xi_{2}<\beta,
$$

and

$$
\Im_{r}(\xi, \alpha, \xi, q), \text { with } \alpha<\xi_{1} \text {, }
$$

are bounded uniformly as to $\xi, \nu$ and $n$ if $q \geqq 0$, and if $q>0$ they approach zero uniformly as to $\xi$ and $\nu$, as $n \rightarrow \infty$.

Since in the identity

$$
e^{\lambda \Gamma_{r}(\beta, \xi)} \equiv e^{\lambda \Gamma_{r}\left(\beta, \xi_{2}\right)} \cdot e^{\lambda \Gamma_{r}\left(\xi_{2}, \xi\right)},
$$

the final exponential is bounded on the $\operatorname{arcs} \gamma_{n r}$, as is shown by the evaluations (24.21), the matrix (25.6) is in fact of the form (25.4) with $\alpha=\xi_{2}$. Similarly the matrix (25.7) is of the form (25.4) with $\beta=\xi_{1}$. The assertions therefore follow from the Lemma 2.

LEMMA 4. For $\alpha \leqq \xi_{1}<\xi_{2} \leqq \beta$ the matrices

$$
\int_{\xi_{1}}^{\xi_{2}} \Im_{r}(\xi, \alpha, \xi, 1) d \xi
$$

and

$$
\int_{\xi_{1}}^{\xi_{2}} \Im_{r}(\beta, \xi, \xi, 1) d \xi,
$$

approach $\mathrm{D}$ as $n \rightarrow \infty$, uniformly as to $\xi_{1}, \xi_{2}$ and $\nu$.

By virtue of the relations (24.21) and (25.1), the elements of the matrix (25.8) are respectively dominated by those of the sum

$$
\sum_{j=0}^{1} \int_{\xi_{1}}^{\xi_{2}} \int_{0}^{\omega_{r-1-j}} \exp \left\{-\Lambda_{n}\left|\Gamma_{r}(\xi, \alpha)\right| \sin \theta\right\}\left(\rho_{i j}\right) d \theta d \xi .
$$


These latter are, however, in turn uniformly dominated by the elements of the matrix sum, which, with any suitably small positive $\epsilon$, is given by the formula

$$
\begin{aligned}
\sum_{j=0}^{1}\left\{\int_{\alpha}^{\alpha+\epsilon}\right. & \int_{0}^{\omega_{r-1-i}}\left(\rho_{i j}\right) d \theta d \xi . \\
& \left.+\int_{\alpha+\epsilon}^{\beta} \int_{0}^{\omega_{r-1-i}} \exp \left\{-\Lambda_{n}\left|\Gamma_{r}(\alpha+\epsilon, \alpha)\right| \frac{\sin \omega_{r-1-i}}{\omega_{r-1-i}} \theta\right\}\left(\rho_{i j}\right) d \theta d \xi\right\} .
\end{aligned}
$$

In these matrices the elements of the first two may be made arbitrarily small by the choice of $\epsilon$, and those of the remaining ones are then arbitrarily small when $n$ exceeds some specific value, as is shown by explicit integrations. The convergence of the matrix (25.8) thus follows, and a similar argument establishes the fact for the matrix (25.9).

LEMMA 5. If $\Re(\xi, \nu, n)$ is any matrix such that:

(i) its elements are uniformly bounded;

(ii) for $\alpha \leqq \xi_{1}<\xi_{2} \leqq \beta$ the matrix

$$
\int_{\xi_{1}}^{\xi_{2}} \Re(\xi, \nu, n) d \xi
$$

approaches $\mathrm{D}$ as $n \rightarrow \infty$, uniformly as to $\xi_{1}, \xi_{2}$ and $\nu$; the relation

$$
\lim _{n \rightarrow \infty} \int_{\alpha}^{\beta} \Re(\xi, \nu, n) \mathfrak{f}(\xi) d \xi=0,
$$

maintains uniformly as to $\nu$, for every vector $\mathfrak{f}(\xi)$ whose components are integrable over the interval $(\alpha, \beta)$.

This is an immediate consequence of a familiar general convergence theorem $\left({ }^{19}\right)$.

LEMMA 6. If $\Re(\xi, \nu, n)$ is any matrix such that:

(i) for $\alpha \leqq \xi_{1}<\xi_{2} \leqq \beta$, the elements of the matrix (25.10) are bounded uniformly as to $\xi_{1}, \xi_{2}, \nu$, and $n$;

(ii) for $\alpha<\xi_{1}<\xi_{2}<\beta$, the matrix (25.10) approaches $\mathfrak{o}$ as $n \rightarrow \infty$, uniformly as to $\nu$;

(iii) for $\alpha<\xi_{2}<\beta$

$$
\lim _{n \rightarrow \infty} \int_{\alpha}^{\xi_{2}} \Re(\xi, \nu, n) d \xi=\mathfrak{R}_{\alpha}(\nu)
$$

(iv) for $\alpha<\xi_{1}<\beta$

(19) E. W. Hobson, The theory of functions of a real variable, Cambridge University Press, Vol. II, 1926, p. 422. 


\section{the relation}

$$
\lim _{n \rightarrow \infty} \int_{\xi_{1}}^{\beta} \Re(\xi, \nu, n) d \xi=\mathfrak{R}_{\beta}(\nu) ;
$$

$$
\lim _{n \rightarrow \infty} \int_{\alpha}^{\beta} \Re(\xi, \nu, n) \mathfrak{f}(\xi) d \xi=\mathfrak{R}_{\alpha} \mathfrak{f}(\alpha+)+\mathfrak{R}_{\beta} \mathfrak{f}(\beta-),
$$

maintains for every vector $f(\xi)$ whose components are of bounded variation on the interval $(\alpha, \beta)$

This is an evident formulation of a familiar theorem in singular integrals $\left({ }^{20}\right)$.

LEMMA 7. If $\Omega(\xi, \nu, n)$ is any matrix which fulfills the specifications (i) and (ii) of Lemma 6 , the relation

$$
\lim _{n \rightarrow \infty} \int_{\alpha}^{\beta} \Re(\xi, \nu, n) \mathfrak{f}(\xi) d \xi=\mathfrak{o},
$$

maintains uniformly as to $\nu$, for every vector $f(\xi)$ whose components are of bounded variation on the interval $(\alpha, \beta)$, and for which $\mathfrak{f}(\alpha+)=0, f(\beta-)=0$.

The argument by which the Lemma 6 is established, serves also to prove the assertion here, the conditions (iii) and (iv) of the Lemma 6 being dispensable because of the vanishing limits of the vectors $f(\xi)$ concerned, at $\xi=\alpha$ and $\xi=\beta$.

On the basis of these lemmas an analysis of the expressions (24.15) is to be given in the remaining discussion. Consistent with the prime purport of this, which is ultimately to establish summability of the expansions by the means $B$, it will be assumed henceforth that all vectors $f(\xi)$ and $\mathfrak{f}^{(j, l)}$ which are brought into question are independent of $\nu$, and that the vectors $f(\xi)$, moreover, all have components that are integrable over the interval $(0,1)$. The point $x$ at which an expansion is considered will always be regarded as fixed. Since the analysis which applies when $x$ is an end point of the basic interval $(0,1)$ differs materially from that which is applicable when $x$ is an interior point, these cases will be separately discussed,- - the latter in $\$ \S 26$ and 27 , and the former finally in $\$ 28$.

26. The convergence of the vector $8_{0}(x, n)$, when $0<x<1$. With the use of the evaluations (24.2) of the matrices $\Im^{(r)}(x, \xi)$, the formula for the vector $\xi_{0}(x, n)$, as it is given by the first one of the relations (24.15), is found, after the collection of similar integrals over abutting arcs of integration, to be expressible in the form

$$
\S_{0}(x, n)=\frac{1}{2 \pi i} \int_{0}^{x} \Re^{(0)}(\xi, n) \mathfrak{f}(\xi) d \xi+\frac{1}{2 \pi i} \int_{x}^{1} \Re^{(1)}(\xi, n) \mathfrak{f}(\xi) d \xi,
$$

$\left.{ }^{20}\right)$ Cf. Hobson, loc. cit. pp. 446-448. 
the matrices $\Omega^{(h)}(\xi, n)$ being given explicitly by the formulas

$$
\Re^{(0)}(\xi, n) \equiv-\sum_{r=1}^{2} \int_{\gamma_{n r}} \mathfrak{V}(x) \Im_{2 r} \mathfrak{Z}^{-1}(\xi) \Re(\xi) d \lambda,
$$

and

$$
\Re^{(1)}(\xi, n) \equiv \sum_{r=3}^{\beta} \int_{\gamma_{n r}} \mathfrak{Y}(x) \Im_{2 r} \mathfrak{Y}^{-1}(\xi) \Re(\xi) d \lambda .
$$

Consider the matrix $\Omega^{(0)}(x, \xi)$. Since by the formula (4.8) the matrix $\mathfrak{V}(x)$ is factorable in the manner $\mathfrak{P}(x) \mathfrak{E}(x)$, whereas the identities

$$
\mathbb{E}(x) \Im_{2 r} \mathbb{E}^{-1}(\xi) \equiv e^{\lambda \Gamma_{r}(x, \xi)} \Im_{2 r}, \quad r=1,2,
$$

maintain, as may be easily verified, it is seen that the formula (26.2) is given somewhat more explicitly by the form

$$
\Re^{(0)}(\xi, n)=-\sum_{r=1}^{2} \int_{\gamma_{n r}} e^{\lambda \Gamma_{r}(x, \xi)} \mathfrak{B}(x) \Im_{2 r} \mathfrak{P}^{-1}(\xi) \Re(\xi) d \lambda .
$$

Now by the formula (4.9) the matrix $\mathfrak{P}(x)$, and hence also its inverse, differs from the unit matrix by a term which is uniformly of the order of $1 / \lambda$. Aside from its scalar exponential factor, the integrand shown in the relation (26.5) is, therefore, of the form

$$
\Im_{2 r} \Re(\xi)+(1 / \lambda) \mathfrak{M}(\xi, \lambda) .
$$

With the use of the relations

$$
\Im_{2 r} \Re(\xi) \equiv \Gamma_{r}^{\prime}(\xi) \Im_{2 r}
$$$$
r=1,2 \text {, }
$$

which follow from the fact that the functions $r_{j}(\xi)$ which are elements of the matrix $\Re(\xi)$ can also be expressed respectively as $\Gamma_{j}^{\prime}(\xi)$, the complete integrands in the formula (26.5) are, therefore, seen to be of the structure

$$
e^{\lambda \Gamma_{r}(x, \xi)} \Gamma_{r}^{\prime}(\xi) \Im_{2 r}+\Im_{r}(x, \xi, \xi, 1) \text {. }
$$

An integration with respect to $\xi$ accordingly yields the relation

$$
\begin{aligned}
\int_{\xi_{1}}^{\xi_{2}} \AA^{(0)}(\xi, n) d \xi=\sum_{r=1}^{2}\left\{\int_{\gamma_{n r}} e^{\lambda \Gamma_{r}\left(x, \xi_{2}\right)} \Im_{2 r} \frac{d \lambda}{\lambda}\right. & -\int_{\gamma_{n r}} e^{\lambda \Gamma_{r}\left(x, \xi_{1}\right)} \Im_{2 r} \frac{d \lambda}{\lambda} \\
& \left.-\int_{\xi_{1}}^{\xi_{2}} \Im_{r}(x, \xi, \xi, 1) d \xi\right\} .
\end{aligned}
$$

Let $x$ now be fixed upon as any point in the interior of the interval $(0,1)$ in some neighborhood of which the components of the generating vector $f(\xi)$ are of bounded variation. With a suitable determination of $\epsilon$ as a positive constant, the neighborhood in question contains the interval $(x-\epsilon, x+\epsilon)$. It will be supposed in the following, that an $\epsilon$ has been determined upon which 
fulfills this condition. Then, on the one hand, if the points $\xi_{3}, \xi_{1}$, and $\xi_{2}$ lie upon the interval $(0, x-\epsilon)$, the integrals in the formula (26.5) are of the form (25.6) with $q=0$ and $\beta=x$, whereas each integral in the formula (26.7) is either of the form (25.6) with $q=1$, or of the form (25.9). It follows from the Lemmas 3 and 4 that the matrix $\Omega^{(0)}(\xi, n)$ fulfills the hypotheses of the Lemma 5, relative to the interval in question, and hence that

$$
\lim _{n \rightarrow \infty} \int_{0}^{x-\epsilon} \Re^{(0)}(\xi, n) \mathfrak{f}(\xi) d \xi=0 .
$$

On the other hand, if the points $\xi_{1}$ and $\xi_{2}$ are taken to lie upon the interval $(x-\epsilon, x)$, it is found similarly by the use of the Lemmas 1,2, and 4, that the matrix $\Omega^{(0)}(\xi, n)$ fulfills the hypotheses of the Lemma 6, with $\alpha=x-\epsilon, \beta=x$, and with $\mathfrak{R}_{\alpha} \equiv \mathrm{D}, \mathfrak{R}_{\beta} \equiv \pi i \Im$. It follows, therefore, that

$$
\lim _{n \rightarrow \infty} \int_{x \rightarrow \epsilon}^{x} \Re^{(0)}(\xi, n) \mathfrak{f}(\xi) d \xi=\pi i f(x-) .
$$

If the consideration is now turned to the matrix $\Omega^{(1)}(\xi, n)$ with $x<\xi$, the reasoning given may be essentially repeated. It is found, thus, on the basis of the relations

$$
\begin{array}{rlr}
\mathfrak{E}(x) \Im_{2 r} \mathcal{E}^{-1}(\xi) & \equiv e^{\lambda \Gamma_{r}(\xi, x)} \Im_{2 r}, & \\
\Im_{2 r} \Re(\xi) & \equiv-\Gamma_{r}^{\prime}(\xi) \Im_{2 r}, & r=3,4,
\end{array}
$$

that

$$
\lim _{n \rightarrow \infty} \int_{x+\epsilon}^{1} \Re^{(1)}(\xi, n) \mathfrak{f}(\xi) d \xi=0 .
$$

and that

$$
\lim _{n \rightarrow \infty} \int_{x}^{x+\epsilon} \Re^{(1)}(\xi, n) \mathfrak{f}(\xi) d \xi=\pi i \mathfrak{f}(x+) .
$$

The convergence and limiting values of the terms of the formula (26.1) have thus been established, the results admitting of summary in the following form.

The vector $\xi_{0}(x, n)$ converges as $n \rightarrow \infty$ to the value

$$
(1 / 2)\{f(x-)+f(x+)\} \text {, }
$$

at every point $x$ which is in the interior of the interval $(0,1)$ and in some neighborhood of which the components of the generating vector $f(\xi)$ are of bounded variation.

27. The summability of the expansions at interior points of the interval $(0,1)$. By the second one of the formulas (24.15) the product of the vector $\varepsilon_{1}(x, \nu, n)$ by $\nu$ is expressible in the form

$$
\nu 8_{1}(x, \nu, n)=\int_{0}^{1} \Re(\xi, \nu, n) \mathfrak{f}(\xi) d \xi,
$$


with the kernel matrix $\Re(\xi, \nu, n)$ given by the relation

$$
\mathfrak{R}(\xi, \nu, n) \equiv \frac{-1}{2 \pi i} \sum_{r=1}^{4} \int_{C_{n r}} \mathfrak{V}_{r}(x)\left\{\nu \mathfrak{l}_{r}(\lambda, \nu)\right\} \mathfrak{A}_{r}(\lambda, \nu) \mathfrak{V}_{r+2}^{-1}(\xi) \Re(\xi) d \lambda .
$$

It was observed in $\$ 24$, moreover, that each matrix which appears in any integrand of this formula (27.2), has elements that are uniformly bounded over the range of integration concerned, provided the parameter $\nu$ is restricted to the respective path segment $\left(\nu_{0}, \nu_{n}\right)$. Under this restriction upon $\nu$, which is to be imposed and maintained throughout this discussion, the integrands of the formula (27.2) are thus all of the type $\mathfrak{M}(\xi, \lambda, \nu)$, as that has been defined in $\$ 25$. A somewhat more explicit determination of the structure of these integrands is requisite, and is obtainable as follows.

By the formula (24.9), the matrices $\mathfrak{D}_{r}(x)$ are found to have, for the several indices $r$, the forms given by the table:

\begin{tabular}{|c|c|}
\hline$r$ & $\mathfrak{V}_{r}(x, \lambda)$ \\
\hline 1 & $e^{\lambda \Gamma_{1}(x, 0)} \mathfrak{B}(x) \Im_{2}+e^{\lambda \Gamma_{2}(x, 0)} \mathfrak{B}(x) \Im_{4}$ \\
\hline 2 & $e^{\lambda \Gamma_{3}(1, x)} \mathfrak{B}(x) \Im_{2}+e^{\lambda \Gamma_{2}(x, 0)} \mathfrak{B}(x) \Im_{4}$ \\
\hline 3 & $e^{\lambda \Gamma_{3}(1, x)} \mathfrak{B}(x) \Im_{2}+e^{\lambda \Gamma_{4}(1, x)} \mathfrak{B}(x) \Im_{4}$ \\
\hline 4 & $e^{\lambda \Gamma_{1}(x, 0)} \mathfrak{B}(x) \Im_{2}+e^{\lambda \Gamma_{4}(1, x)} \mathfrak{B}(x) \Im_{4}$ \\
\hline
\end{tabular}

If these forms are substituted into the relation (27.2), and thereupon the integrals involving any specific exponential over contiguous arcs of integration are collected, it is found that the result may be written in the manner

$$
\mathfrak{\Omega}(\xi, \nu, n)=\sum_{r=1}^{2} \int_{\gamma_{n r}} e^{\lambda \Gamma_{r}(x, 0)} \mathfrak{M}(\xi, \lambda, \nu) d \lambda+\sum_{r=3}^{4} \int_{\gamma_{n r}} e^{\lambda \Gamma_{r}(1, x)} \mathfrak{M}(\xi, \lambda, \nu) d \lambda .
$$

Specifically the matrix indicated here by $\mathfrak{M}(\xi, \lambda, \nu)$ over any semicircle $\gamma_{n}$, is identifiable as the product

$$
\left[\mathfrak{P}(x) \Im_{2 r}\left\{\nu \mathfrak{U}_{r-j}\right\} \mathfrak{A}_{r-j} \mathfrak{V}_{r+1-j}^{-1}(\xi)\right] \Re(\xi) \text { on the arc } C_{n, r-j}, \quad j=0,1 .
$$

Consider any matrix $\mathbb{Z}(x, \lambda, \nu)$ which fulfills a relation

$$
\mathbb{Z}(\xi) \mathfrak{Y}(\xi) \equiv \mathfrak{c},
$$

in which $(\mathcal{E}$ is a matrix that is constant as to $\xi$. It may be deduced from the equation (4.1), then, that $\mathbb{Z}(\xi)$ is a solution of the adjoint differential equation

$$
\mathfrak{Z}^{\prime}(\xi)=-\mathfrak{Z}(\xi)\{\lambda \Re(\xi)+\mathfrak{Q}(\xi)\},
$$

and from this it follows that with any choice of $\xi_{1}$ and $\xi$ on the interval $(0,1)$ 


$$
\int_{\xi_{1}}^{\xi} \mathfrak{Z}(\xi) \Re(\xi) d \xi=\frac{-1}{\lambda}\left\{\mathfrak{Z}(\xi)-\mathfrak{Z}\left(\xi_{1}\right)+\int_{\xi_{1}}^{\xi} \mathfrak{Z}(\xi) \mathfrak{Q}(\xi) d \xi\right\} .
$$

This formula is applicable to the integration of the relation (27.4). Since the matrix products enclosed within the square brackets in the expressions (27.5) are each of the type prescribed by the designation 3 , the integration of these expressions is given by the formula (27.8). Since, furthermore, each matrix thus designated by 3 is of the type denotable by $\mathfrak{M}(\xi, \lambda, \nu)$, the entire right-hand member of any resulting relation (27.8) is clearly of the form $(1 / \lambda) \mathfrak{M}(\xi, \lambda, \nu)$, uniformly as to the choice of $\xi_{1}$, on the interval $(0,1)$. The formula (27.2) leads, therefore, also to the relations

$$
\begin{aligned}
\int_{\xi_{1}}^{\xi_{2}} \Re(\xi, \nu, n) d \xi= & \sum_{r=1}^{2} \int_{\gamma_{n r}} e^{\lambda \Gamma_{r}(x, 0)} \mathfrak{M}\left(\xi_{2}, \lambda, \nu\right) \frac{d \lambda}{\lambda} \\
& +\sum_{r=3}^{4} \int_{\gamma_{n r}} e^{\lambda \Gamma_{r}(1, x)} \mathfrak{M}\left(\xi_{2}, \lambda, \nu\right) \frac{d \lambda}{\lambda} .
\end{aligned}
$$

The integrals in the right-hand members of the formulas (27.4) and (27.9) may now all be recognized as being of the forms (25.4) with $q$, respectively, equal to either 0 or 1 . It follows from this by the Lemma 2 that the matrix (27.2) fulfills the hypotheses of the Lemma 5. By that lemma, then, the relation (27.1) leads to the conclusion that

$$
\lim _{n \rightarrow \infty}\left\{\nu \varepsilon_{1}(x, \nu, n)\right\}=\mathfrak{o}
$$

uniformly as to $\nu$ on the segment $\left(\nu_{0}, \nu_{n}\right)$.

The analysis which has thus been given for the second one of the formulas (24.15) may be applied equally well, and in a wholly similar manner to the third one of these formulas. It yields in this instance an evaluation of the form

$$
\begin{aligned}
\nu \varepsilon_{2}(x, \nu, n)= & \sum_{l=0}^{1-1} \sum_{h=0}^{1}\left\{\sum_{r=1}^{2} \int_{\gamma_{n r}} e^{\lambda \Gamma_{r}(x, 0)} \mathfrak{M}(\lambda, \nu) \frac{d \lambda}{\lambda^{l+1}} \mathfrak{f}^{(h, l)}\right. \\
& \left.+\sum_{r=3}^{4} \int_{\gamma_{n r}} e^{\lambda \Gamma_{r}(1, x)} \mathfrak{M}(\lambda, \nu) \frac{d \lambda}{\lambda^{l+1}} \mathfrak{f}^{(h, l)}\right\}
\end{aligned}
$$

and inasmuch as each integrand on the right of this equality is of the type (25.4) with $q>0$, it follows from the Lemma 2 that irrespective of the vectors $f^{(h, l)}$ involved,

$$
\lim _{n \rightarrow \infty}\left\{\nu \mathbb{8}_{2}(x, \nu, n)\right\}=\mathfrak{v}
$$

uniformly as to $\nu$ on the segment $\left(\nu_{0}, \nu_{n}\right)$.

In virtue of the conclusions (27.10) and (27.12), it may evidently be in- 
ferred that there exists a positive monotonic sequence of constants

$$
\epsilon_{1}, \epsilon_{2}, \epsilon_{3}, \cdots
$$

which converges to zero, and which is furthermore such that for each index $n$, and for all values of $\nu$ on the respective path segment $\left(v_{0}, \nu_{n}\right)$, the relations

$$
\left|\nu 8_{1}(x, \nu, n)+\nu 8_{2}(x, \nu, n)\right|<\epsilon_{n},
$$

are fulfilled. On the path of $\nu$ let the sequence of points

$$
\nu_{1}^{\prime}, \nu_{2}^{\prime}, \nu_{3}^{\prime}, \cdots
$$

be determined now so that

(i) $\nu_{n}^{\prime}$ lies on the segment $\left(\nu_{0}, \nu_{n}\right)$,

(ii)

$$
\lim _{n \rightarrow \infty} \nu_{n}^{\prime}=0 \text {, }
$$

and

$$
\lim _{n \rightarrow \infty} \frac{\epsilon_{n}}{\nu_{n}^{\prime}}=0
$$

The determination of such a sequence is clearly possible. Since the inequalities (27.13) are, then, in particular fulfilled when $\nu=\nu_{n}{ }^{\prime}$, it follows from them, together with the relation (27.16), that

$$
\lim _{n \rightarrow \infty}\left\{g_{1}\left(x, \nu_{n}^{\prime}, n\right)+\xi_{2}\left(x, \nu_{n}^{\prime}, n\right)\right\}=0 .
$$

This result, taken in conjunction with that of $\$ 26$, permits of the conclusion which follows.

THEOREM. The expansion generated by an arbitrary integrable vector $\mathrm{f}(\xi)$, and an arbitrary set of associated vectors $\mathrm{f}^{(h, l)}, h=0,1 ; l=1,2,3, \cdots,\left(\tau_{1}-1\right)$; is summable by the means $B$ to the value $(1 / 2)\{f(x-)+f(x+)\}$ at every interior point of the basic interval in some neighborhood of which the components of the vector $f(\xi)$ are of bounded variation.

28. The summability of the expansions at the end points of the interval. The reasoning of the two immediately preceding sections depends explicitly upon the relation $0<x<1$, and is essentially inadequate when the point at which the expansion is considered is an end point of the basic interval. At these points, $x=0$, and $x=1$, therefore, distinct considerations are requisite. Such are to be given in the following, it being assumed throughout their course, firstly, that the given boundary problem has, if necessary, been adjusted in the manner described in $\$ 3$, so that the relations (3.2) maintain; and secondly, that only such expansions are brought into question as have generating vectors $f(\xi)$ whose components are of bounded variation in some 
right-hand neighborhood of $\xi=0$ and in some left-hand neighborhood of $\xi=1$. A positive constant $\epsilon$ may be determined in each case, then, so that the boundedness of variation assumed is maintained over each one of the intervals $(0, \epsilon)$ and $(1-\epsilon, 1)$. It will be supposed that $\epsilon$ has been so determined. The pair of intervals $(0, \epsilon),(1-\epsilon, 1)$ will briefly be designated by the symbol $\Delta$, and it shall be understood that the designations $f(0)$ and $f(1)$ signify the limiting values $f(0+)$ and $f(1-)$, respectively.

As a function of $\xi$ the Green's matrix $\mathcal{S}(x, \xi, \lambda, \nu)$ is a solution of the differential equation (27.7), as may be seen at once from its definition (22.1). It is found in virtue of this that the relation

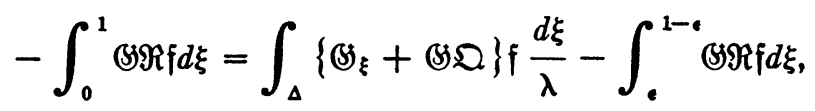

maintains, and furthermore that every differentiable vector $g(\xi)$ satisfies the identity

$$
\begin{aligned}
& 0 \equiv \int_{0}^{1} \frac{\partial}{\partial \xi}\{\leftrightarrow \mathfrak{g}\} \frac{d \xi}{\lambda}-\int_{\Delta}\left\{\xi_{\xi} \mathfrak{g}+\mathfrak{S g}_{\mathfrak{g}}^{\prime}\right\} \frac{d \xi}{\lambda} \\
& +\int_{0}^{1-\epsilon}\left\{\lambda \circlearrowleft \Re g+\mathfrak{S} \mathfrak{Q} g-\mathfrak{S g}^{\prime}\right\} \frac{d \xi}{\lambda} \text {. }
\end{aligned}
$$

The specific vector for which this identity is to be utilized, and to which the designation $\mathfrak{g}(\xi)$ will hereafter be restricted, is the following one:

$$
\mathfrak{g}(\xi) \equiv \xi \mathfrak{f}(1)+(1-\xi) \mathfrak{f}(0) .
$$

If the respective members of the identities (28.1) and (28.2) are added, and their sum is integrated over the contour $C_{n}$, the result is an evaluation of first integral in the formula (22.8). With a suitable grouping of the terms over the several ranges of integration, that formula is, therefore, found to be expressible in the form

$$
\mathcal{B}(x, \nu, n)=f(x)+\sum_{k=0}^{5} 8_{0 k}(x, \nu, n)
$$

with

and

$$
\begin{aligned}
\mathfrak{B}_{00} \equiv & -\mathfrak{f}(x)+\frac{1}{2 \pi i} \int_{0}^{1} \int_{C_{n}} \frac{\partial}{\partial \xi}\{\mathfrak{G g}\} \frac{d \lambda}{\lambda} d \xi \\
& +\frac{1}{2 \pi i} \int_{C_{n}} \mathfrak{D}(x) \mathfrak{D}^{-1}\left\{\mathfrak{B}^{(0,0)}(\lambda) \mathfrak{f}(0)+\mathfrak{B}^{(1,0)}(\lambda) \mathfrak{f}(1)\right\} d \lambda,
\end{aligned}
$$

$$
\mathfrak{B}_{01} \equiv \sum_{l=1}^{r_{1}-1} \frac{1}{2 \pi i} \int_{C_{n}} \mathfrak{D}(x) \mathfrak{D}^{-1}\left\{\mathfrak{B}^{(0, l)}(\lambda) \mathfrak{f}^{(0, l)}+\mathfrak{B}^{(1, l)}(\lambda) \mathfrak{f}^{(1, l)}\right\} d \lambda
$$


and with the remaining terms expressible through the matrices

$$
\begin{aligned}
& \Re^{(1)}(x, \xi, \nu, n) \equiv \frac{1}{2 \pi i} \int_{C_{n}}\left(\xi(x, \xi, \lambda, \nu) \frac{d \lambda}{\lambda},\right. \\
& \Re^{(2)}(x, \xi, \nu, n) \equiv \frac{1}{2 \pi i} \int_{C_{n}}\left(\xi \xi(x, \xi, \lambda, \nu) \frac{d \lambda}{\lambda},\right. \\
& \Re^{(3)}(x, \xi, \nu, n) \equiv \frac{1}{2 \pi i} \int_{C_{n}}(\mathcal{S}(x, \xi, \lambda, \nu) \Re(\xi) d \lambda,
\end{aligned}
$$

by the formulas

$$
\begin{aligned}
& \mathbb{8}_{02}=\int_{0}^{1-\bullet} \Re^{(1)}\left\{\mathfrak{Q} g-\mathfrak{g}^{\prime}\right\} d \xi, \\
& \boldsymbol{g}_{03}=\int_{\Delta} \mathfrak{R}^{(1)}\left\{\mathfrak{Q} \mathfrak{f}-\mathfrak{g}^{\prime}\right\} d \xi,
\end{aligned}
$$

and

$$
\begin{aligned}
& \mathbb{8}_{04}=\int_{\Delta} \Omega^{(2)}\{\mathfrak{f}-\mathfrak{g}\} d \xi, \\
& \mathbb{8}_{05}=-\int_{0}^{1-\mathfrak{G}} \Omega^{(8)}\{\mathfrak{f}-\mathfrak{g}\} d \xi .
\end{aligned}
$$

Consider the formula (28.5). If the indicated integration as to $\xi$ therein is performed, and the expression $f(x)$ is replaced by its equivalent

$$
\frac{1}{2 \pi i} \int_{c_{n}} f(x) \frac{d \lambda}{\lambda},
$$

the formula is seen to be alternatively

$$
\begin{aligned}
\mathbb{B}_{00}=\frac{1}{2 \pi i} \int_{C_{n}}\{\mathfrak{S}(x, & 1-) \mathfrak{f}(1)-\mathfrak{S}(x, 0+) \mathfrak{f}(0)-\mathfrak{f}(x) \\
& \left.+\mathfrak{W}(x) \mathfrak{D}^{-1}\left[\lambda \mathfrak{B}^{(0,0)}(\lambda) \mathfrak{f}(0)+\lambda \mathfrak{B}^{(1,0)}(\lambda) \mathfrak{f}(1)\right]\right\} \frac{d \lambda}{\lambda} .
\end{aligned}
$$

Now the definition (22.1) of the Green's matrix leads to the evaluations

$$
\begin{array}{lr}
\mathfrak{S}(0,0+)= & -\Im+\mathfrak{V}(0) \mathfrak{D}^{-1} \mathfrak{B}^{(0)}(\lambda), \\
\mathfrak{S}(0,1-)= & -\mathfrak{D}(0) \mathfrak{D}^{-1} \mathfrak{B}^{(1)}(\lambda), \\
\mathfrak{S}(1,0+)= & \mathfrak{V}(1) \mathfrak{D}^{-1} \mathfrak{B}^{(0)}(\lambda), \\
\mathfrak{S}(1,1-)= & \Im-\mathfrak{V}(1) \mathfrak{D}^{-1} \mathfrak{B}^{(1)}(\lambda),
\end{array}
$$

whereas it follows from the relations (20.1), with $\lambda^{\prime}$ and $\lambda^{\prime \prime}$ identified, respectively, as $\lambda$ and 0 , that

$$
\lambda \mathfrak{B}^{(h, 0)}(\lambda)=\mathfrak{B}^{(h)}(\lambda)-\mathfrak{B}^{(h)}(0), \quad h=0,1 .
$$


In virtue of these substitutions the formula (28.10) reduces, irrespective of whether $x=0$ or $x=1$, to the form

$$
\Xi_{00}=\frac{-1}{2 \pi i} \int_{C_{n}} \mathfrak{D}(x) \mathfrak{D}^{-1} \frac{d \lambda}{\lambda}\left\{\mathfrak{B}^{(0)}(0) \mathfrak{f}(0)+\mathfrak{B}^{(1)}(0) \mathfrak{f}(1)\right\},
$$

and this leads finally, through the evaluations

$$
\mathfrak{D}(x) \mathfrak{D}^{-1}(\lambda)=\mathfrak{D}_{r}(x) \mathfrak{U}_{r}\left\{\lambda^{-r_{1}} \mathfrak{\Im}_{2}+\lambda^{-r_{2}} \mathfrak{G}_{4}\right\}, \quad r=1,2,3,4,
$$

to the relation

(28.13) $\nu \S_{00}=\frac{-1}{2 \pi i} \sum_{r=1}^{4} \sum_{j=1}^{2} \int_{C_{n r}} \mathfrak{V}_{r}(x)\left\{\nu \mathfrak{U}_{r}\right\} \Im_{2 j} \frac{d \lambda}{\lambda^{r_{j}+1}}\left\{\mathfrak{B}^{(0)}(0) \mathfrak{f}(0)+\mathfrak{B}^{(1)}(0) \mathfrak{f}(1)\right\}$.

The transformation of the formula (28.6) is more direct. Through the mere use of the second one of the equations (24.11), it yields, namely the relation

$$
\nu \mathbb{8}_{01}=\frac{1}{2 \pi i} \sum_{l=1}^{1-1} \sum_{r=1}^{4} \sum_{h=0}^{1} \int_{C_{n r}} \mathfrak{V}_{r}(x)\left\{\nu \mathfrak{U}_{r}\right\} \mathfrak{W}^{(h, l)} \frac{d \lambda}{\lambda^{l+1}} \mathfrak{f}^{(h, l)} .
$$

Under the restriction of the parameter $\nu$ to the path segment $\left(\nu_{0}, \nu_{n}\right)$, each matrix factor which appears in an integrand in the formulas (28.13) and (28.14) has been seen to be uniformly bounded over the range of integration for which it is involved. Due to the relations (3.2), and by the Lemma 1 , therefore, it may be concluded that

$$
\begin{aligned}
& \lim _{n \rightarrow \infty}\left\{\nu 8_{00}(x, \nu, n)\right\}=0, \\
& \lim _{n \rightarrow \infty}\left\{\nu 8_{01}(x, \nu, n)\right\}=0,
\end{aligned}
$$

uniformly as to $\nu$ on the segment $\left(\nu_{0}, \nu_{n}\right)$.

On the basis of the first one of the formulas (24.11), and by considerations which are now familiar, it is found that both when $x=0$ and when $x=1$ the definitions (28.7) assure for the matrices $\nu \Omega^{(1)}(x, \xi, \nu, n)$ and $\nu \Omega^{(3)}(x, \xi, \nu, n)$ the forms

$$
\begin{array}{r}
(28.16) \nu \Re^{(3-2 j)}=\sum_{r=1}^{2} \int_{\gamma_{n r}} e^{\lambda \Gamma_{r}(1, \xi)} \mathfrak{M}(\xi, \lambda, \nu) \frac{d \lambda}{\lambda^{j}}+\sum_{r=3}^{4} \int_{\gamma_{m r}} e^{\lambda \Gamma_{r}(\xi, 0) \mathfrak{M}(\xi, \lambda, \nu) \frac{d \lambda}{\lambda^{j}},} \\
j=0,1,
\end{array}
$$

provided $\nu$ is restricted to range between $\nu_{0}$ and $\nu_{n}$. For any choice of $\xi_{1}$ and $\xi_{2}$, moreover,

$$
\int_{\xi_{1}}^{\xi_{2}} \nu \Re^{(2)}(x, \xi) d \xi=\nu \Re^{(1)}\left(x, \xi_{2}\right)-\nu \Re^{(1)}\left(x, \xi_{1}\right),
$$


and

$$
\begin{aligned}
\int_{\xi_{1}}^{\xi_{2}} \nu \Re^{(3)}(x, \xi,) d \xi= & -\nu \Re^{(1)}\left(x, \xi_{2}\right)+\nu \Re^{(1)}\left(x, \xi_{1}\right) \\
& -\int_{\xi_{1}}^{\xi_{2}} \nu \Re^{(1)}(x, \xi) \mathfrak{Q}(\xi) d \xi,
\end{aligned}
$$

the latter one of these relations following from the fact that the matrix (s) satisfies the differential equation. (27.7).

The Lemmas 1 and 4 applied to the formula (28.16) with $j=1$, show readily that the matrix $\nu \Omega^{(1)}(x, \xi)$ fulfills the hypotheses of the Lemma 5 with $\mathfrak{R}_{\alpha}(\nu) \equiv \mathcal{D}$, and $\mathfrak{R}_{\beta}(\nu) \equiv \mathcal{D}$, and with $(\alpha, \beta)$ as any subinterval of the interval $(0,1)$. Since the vectors $\left\{\mathfrak{Q}(\xi) \mathfrak{g}(\xi)-\mathfrak{g}^{\prime}(\xi)\right\}$ and $\left\{\mathfrak{Q}(\xi) \mathfrak{f}(\xi)-\mathfrak{g}^{\prime}(\xi)\right\}$ have components that are of bounded variation respectively on the interval $(\epsilon, 1-\epsilon)$ and the pair of intervals $\Delta$, it follows from the formulas (28.8) and the Lemma 5 that

$$
\begin{aligned}
& \lim _{n \rightarrow \infty}\left\{\nu \mathbb{8}_{02}(x, \nu, n)\right\}=0, \\
& \lim _{n \rightarrow \infty}\left\{\nu 8_{03}(x, \nu, n)\right\}=0,
\end{aligned}
$$

uniformly as to $\nu$ on the segment $\left(\nu_{0}, \nu_{n}\right)$.

The formulas (28.17) and (28.16) with $j=1$, show readily that the matrix $\nu \Omega^{(2)}(x, \xi)$ fulfills the hypotheses of the Lemma 7 relative to the intervals $\Delta$. The formulas (28.18) and (28.16) with $j=0$, show similarly that the matrix $\nu \Omega^{(3)}(x, \xi)$ fulfills the hypotheses of the Lemma 5 relative to the interval $(\epsilon, 1-\epsilon)$. In virtue of the formulas (28.9), and the fact that the vector $\{f(\xi)-g(\xi)\}$ vanishes at $\xi=0$ and $\xi=1$, it follows, therefore, lastly that

$$
\begin{aligned}
& \lim _{n \rightarrow \infty}\left\{\nu 8_{04}(x, \nu, n)\right\}=0, \\
& \lim _{n \rightarrow \infty}\left\{\nu \varepsilon_{05}(x, \nu, n)\right\}=0,
\end{aligned}
$$

uniformly as to $\nu$ on the segment $\left(\nu_{0}, \nu_{n}\right)$.

The results (28.15), (28.19) and (28.20) evidently insure the existence of a sequence of positive constants $\epsilon_{1}, \epsilon_{2}, \epsilon_{3}, \cdots$ which converges to zero, and which is such that the relations

$$
\left|\nu \sum_{k=0}^{5} 8_{0 k}(x, \nu, n)\right|<\epsilon_{n}, \quad n=1,2,3, \cdots,
$$

maintain, irrespective of how in the $n$th one of them the value of $\nu$ is chosen on the path segment $\left(\nu_{0}, \nu_{n}\right)$. In particular, then, these points may be chosen as the respective members of a sequence (27.14) which fulfills the relations (27.15) and (27.16). For such a choice it is clear that 


$$
\lim _{n \rightarrow \infty} \sum_{k=0}^{5} 8_{0 k}\left(x, \nu_{n}^{\prime}, n\right)=0
$$

and this result yields through the relation (28.4) the following and final conclusion.

THEOREM. The expansion generated by an arbitrary integrable vector $\mathfrak{f}(\xi)$ whose components are of bounded variation in some right-hand neighborhood of the point $\xi=0$, and in some left-hand neighborhood of the point $\xi=1$, is summable by the means $B$ to the vector $f(x)$ at the points $x=0$ and $x=1$.

UNIVERSITY OF WISCONSIN,

Madison, Wis. 